SERVIÇO DE PÓS-GRADUAÇÃO DO ICMC.USP

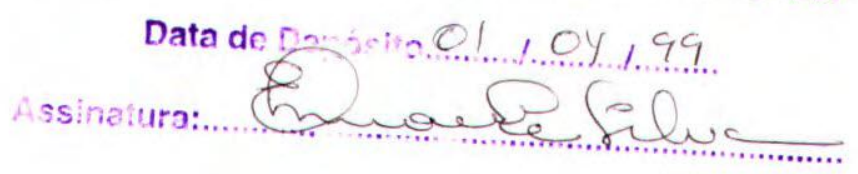

\title{
Sincronização e Homogeneização em Problemas Parabólicos com Condição de Fronteira Não Linear
}

Marcos Roberto Teixeira Primo

Orientador:

Prof. Dr. Alexandre Nolasco de Carvalho

Tese de Doutorado apresentada ao Instituto de Ciências Matemáticas e de Computação, da Universidade de São Paulo, como parte dos requisitos para obtenção do título de "Doutor em Ciências - Área: Matemática".

USP - São Carlos

Março de 1999 
Dedico este trabalho à minha mãe Maria Aparecida Barbosa Teixeira 


\section{Agradecimentos ${ }^{1}$}

Ao Alexandre pelo incentivo, pelos conselhos, pela dedicação, pela calma e pela forma segura e paciente com que sempre nos orientou durante o desenvolvimento deste trabalho.

À minha família: Seu Altino, Branco, Luciana, Aluethi e Eduardo, pela alegria e pelo apoio durante os momentos mais difíceis de todos esses anos, e ao Marcelo que nos faz muita falta.

Aos professores do Departamento de Matemática do ICMC-USP - São Carlos com os quais tive o prazer de me relacionar ao longo desses anos, e a todos os professores do DMA-UEM - Maringá.

À Beth, à Laura e à Marília pelo zelo com que sempre tratam de nossos assuntos acadêmicos.

A todos os funcionários do ICMC-USP - São Carlos, em particular ao pessoal da Biblioteca e ao pessoal do cafezinho. Aos funcionários da UEM - Maringá pela disposição com que sempre nos atenderam.

Gostaria de agradecer também ao pessoal da república: Francisco, Lucimara, Patrícia, Silmar e Willian pela ótima convivência em nossa república, e ao Miro, que nos fez muita falta.

Agradeço, de todo coração, aos amigos que me suportaram e tornaram esses anos mais agradáveis, não citarei nomes aqui, para não cometer injustiça com alguns.

Enfim, agradeço a todos que cooperaram para a realização deste trabalho. Desde já, peço desculpas às pessoas que involuntariamente não foram citadas aqui.

\footnotetext{
${ }^{1}$ Este trabalho teve suporte financeiro parcial do CNPq e da CAPES
} 


\begin{abstract}
In this work, we prove the synchronization of parabolic problems with nonlinear boundary condition and dissipative boundary coupling. By synchronization, we mean that the coordinates of the system (made of the two parabolic equations with nonlinear boundary condition, linearly coupled in the boundary) are close for large times. This is obtained, if the nonlinear terms are approximately the same.

We also prove that the global attractor of a weakly coupled system of parabolic equations with nonlinear boundary conditions, is contained in a neighborhood of the global attractor of an ordinary differential equation, if the diffusivity is large.

To obtain the results above, we employ results of comparison and positivity, to show global existence and existence of global attractors.
\end{abstract}




\section{Resumo}

Neste trabalho, provamos a sincronização de problemas parabólicos com condição de fronteira não linear e com acoplamento dissipativo na fronteira. Por sincronização, entedemos que as coordenadas do sistema (feito pelas equações parabólicas com condição de fronteira não linear, linearmente acopladas na fronteira ) estão próximas para tempos grandes. Isto é obtido, se os termos não lineares são aproximadamente os mesmos.

Provamos também, que o atrator global de um sistema de equações parabólicas fracamente acopladas com condição de fronteira não linear, está contido em uma vizinhança do atrator global de uma equação diferencial ordinária, se o coeficiente de difusão é grande.

Para obtemos os resultados acima, utilizamos resultados de comparação e positividade, para mostrarmos existência global e existência de atratores globais. 


\section{Índice}

Introduçāo 1

1 Unicidade e Existência Local de Soluçōes $\quad 7$

1.1 Escalas de Espaços de Banach . . . . . . . . . . . . . . . . . 9

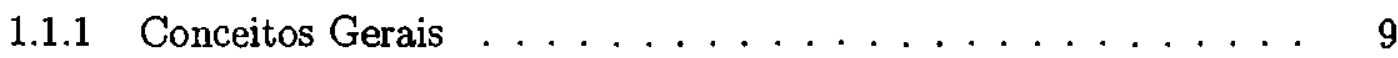

1.1 .2 Escalas de Potência . . . . . . . . . . . . . . . 13

1.1.3 Espaços de Extrapolação . . . . . . . . . . . . . . . 16

1.1 .4 Escalas Duais . . . . . . . . . . . . . . . . . . . . 19

1.1.5 Escalas de Interpolação-Extrapolação . . . . . . . . . . . 24

1.1.6 Semigrupos em Escalas de Interpolação-Extrapolação . . . . . . 25

1.1.7 Um Exemplo . . . . . . . . . . . . . . . . . . . . . 29

1.2 Existência Local de Soluçōes - Resultados Abstratos . . . . . . . . . . 30

1.2.1 Existência de Soluções . . . . . . . . . . . . . . . . 32

1.2 .2 Unicidade de Soluções $\ldots \ldots \ldots \ldots$. . . . . . . . . 37

1.2 .3 Prova do Teorema $1.9 \ldots \ldots \ldots$

1.3 Existência e Unicidade de Soluções para o Problema (1.1) . . . . . . . 41

1.3.1 Existência e Unicidade de Soluçōes em $L^{q}\left(\Omega, \mathbb{R}^{n}\right) \ldots \ldots \ldots .44$

1.3.2 Existência e Unicidade de Soluçōes em $W^{1, q}\left(\Omega, \mathbb{R}^{n}\right) \ldots \ldots .49$

2 Existência Global de Soluçōes e Existência de Atratores Globais 51

2.1 Resultados de Comparação e Positividade . . . . . . . . . . . . 51

2.2 Existência Global e de Atratores Globais . . . . . . . . . . . . . . . 64

2.3 Semicontinuidade Superior de Atratores . . . . . . . . . . . 75

3 Sincronização Através da Fronteira em Problemas Parabólicos com Condição de Fronteira não Linear 
3.1 Sincronização em Problemas Parabólicos . . . . . . . . . . . 80

3.2 Um Exemplo . . . . . . . . . . . . . . . . . . . . . . 89

4 Homogeneização em Problemas Parabólicos com Condição de Fronteira não Linear $\quad 92$

4.1 Enunciado dos Resultados Principais . . . . . . . . . . . . . . . 93

4.2 Prova do Teorema $4.1 \ldots \ldots \ldots \ldots$. . . . . . . . . 97

$\begin{array}{lll}\text { Referências Bibliográficas } & 114\end{array}$ 


\section{Introdução}

O objetivo deste trabalho é mostrar algumas propriedades assintóticas de soluções do seguinte problema parabólico semilinear condiçāo de fronteira não linear:

$$
\left\{\begin{array}{l}
u_{t}(t, x)=D \Delta u(t, x)+F(u(t, x)) \quad t>0, \quad x \in \Omega \\
D \frac{\partial u}{\partial \vec{n}}(t, x)=\lambda G(u(t, x)) \quad t>0, \quad x \in \Gamma
\end{array}\right.
$$

onde

- $u=\left(u_{1}, u_{2}, \cdots, u_{n}\right)^{\top}, n \geq 1$

- $\Omega \subset \mathbb{R}^{N}, N \geq 1$, é um domínio aberto, limitado com fronteira $\Gamma:=\partial \Omega$ suave;

- $\frac{\partial u}{\partial \vec{n}}=\langle\nabla u, \vec{n}\rangle, \vec{n}$ é a normal à $\Gamma$, apontando para fora de $\Omega$;

- $D$ é a seguinte matriz $n \times n$ :

$$
D:=\left[\begin{array}{lllll}
d_{1} & 0 & 0 & \cdots & 0 \\
0 & d_{2} & 0 & \cdots & 0 \\
0 & 0 & d_{3} & \cdots & 0 \\
\vdots & \vdots & \vdots & \ddots & \vdots \\
0 & 0 & 0 & \cdots & d_{n}
\end{array}\right]
$$

com $d_{i}>0$, para todo $i=1,2, \cdots, n$;

- $F=\left(F_{1}, F_{2}, \cdots, F_{n}\right): \mathbb{R}^{n} \rightarrow \mathbb{R}^{n}$ e $G=\left(G_{1}, G_{2}, \cdots, G_{n}\right): \mathbb{R}^{n} \rightarrow \mathbb{R}^{n}$ são duas funções tais que se $u=\left(u_{1}, u_{2}, \cdots, u_{n}\right)$, então

$$
\begin{aligned}
& F_{i}(u)=f_{i}(u), \quad i=1,2, \cdots, n, \\
& G_{1}(u)=g_{1}(u)-k\left(u_{1}-u_{2}\right), \\
& G_{2}(u)=g_{2}(u)+k\left(u_{1}-u_{2}\right), \\
& G_{i}(u)=g_{i}(u), \quad i=3,4, \cdots, n, \text { se } n>2,
\end{aligned}
$$


onde $f_{i}, g_{i}: \mathbb{R}^{n} \rightarrow \mathbb{R}, i=1,2, \cdots n$, são funçōes localmente lipschitzianas;

- $k$ e $\lambda$ são constantes positivas.

Em [5], foi mostrado que o problema

$$
\left\{\begin{array}{l}
u_{t}(t, x)=\Delta u(t, x)+f(u(t, x)) \quad t>0, \quad x \in \Omega, \\
\frac{\partial u}{\partial \vec{n}}(t, x)=g(u(t, x)) \quad t>0, \quad x \in \Gamma,
\end{array}\right.
$$

onde $f, g: \mathbb{R} \rightarrow \mathbb{R}$ são funções localmente lipschitzianas, é localmente bem posto em $W^{1, q}(\Omega)$ e em $L^{q}(\Omega)$. No Capítulo 1 , usaremos a teoria abstrata desenvolvida em [5] para mostrarmos que o problema (1) é localmente bem posto em $W^{1, q}\left(\Omega, \mathbb{R}^{n}\right)$ e em $L^{q}\left(\Omega, \mathbb{R}^{n}\right)$. Para enunciarmos com mais precisão estes resultados, e os principais resultados deste trabalho precisamos antes introduzir alguns conceitos e algumas notaçōes.

Consideremos o operador $A=\operatorname{diag}\left(A_{1}, A_{2}, \cdots, A_{n}\right)$, onde para cada $i=1,2, \cdots, n$, $A_{i}$ é definido da seguinte maneira: sejam $A_{i}: D\left(A_{i}\right) \subset L^{q}(\Omega) \rightarrow L^{q}(\Omega)$, com

$$
\begin{aligned}
& D\left(A_{i}\right)=\left\{\phi \in W^{2, q}(\Omega) ; \frac{\partial \phi}{\partial \vec{n}}=0, \text { em } \Gamma\right\}:=W_{\mathcal{N}}^{2, q}(\Omega), \\
& A_{i} \phi=-d_{i} \triangle \phi
\end{aligned}
$$

e seja $X_{q}^{\alpha}, \alpha \in \mathbb{R}$ e $1<q<\infty$, a escala de espaços de Banach associadas ao operador $A$, no Capítulo 1, seguindo as idéias contidas em [3], daremos uma breve descrição de como construir tal escala de espaços de Banach. Seguindo as idéias contidas em [4] e [5], transformamos o problema (1) em um problema abstrato da forma:

$$
\begin{aligned}
& \dot{x}(t)=-A x(t)+\sum_{i=1}^{p} H_{i}(x(t)), t>0, \\
& x(0)=x_{0},
\end{aligned}
$$

em $X=L^{q}\left(\Omega, \mathbb{R}^{n}\right)$ ou em $X=W^{1, q}\left(\Omega, \mathbb{R}^{n}\right)$, onde $A$ é o operador definido em (4), e $H_{i}, i=1,2, \cdots, p$ são funções adequadas, as quais definiremos precisamente no decorrer deste trabalho. Para obtermos existência e unicidade de soluções, precisamos entretanto impor algumas restrições de crescimento sobre os termos não lineares do problema (1). As condiçoes de crescimentos a seguir, são as mesmas impostas em [4] e em [5]. 
(C1) Para obtermos soluções do problema (1), com dado inicial em $L^{q}\left(\Omega, \mathbb{R}^{n}\right)$, suponhamos que $f_{i}, g_{i}, i=1,2, \cdots, n$ satisfaçam uma relação da forma

$$
|h(u)-h(v)| \leq c|u-v|\left(|u|^{\rho-1}+|v|^{\rho-1}+1\right), u, v \in \mathbb{R}^{n},
$$

com expoentes $\rho_{i}$ e $\overline{\rho_{i}}, i=1,2, \cdots, n$, respectivamente, tais que, com $N \geq 2$,

$$
\rho_{i} \leq \rho_{f}:=1+\frac{2 q}{N} \text { e } \overline{\rho_{i}} \leq \rho_{g}:=1+\frac{q}{N}, i=1,2,
$$

sendo estrita a segunda desigualdade para o caso $N=1$.

(C2) Para obtermos soluçōes do problema (1), com dado inicial em $W^{1, q}\left(\Omega, \mathbb{R}^{n}\right)$, suponhamos que

1. $q>N$,

2. $q=N$ e para todo $\eta>0$, existam constantes $c_{\eta}>0$ tais que $f_{i}$ e $g_{i}$, $i=1,2, \cdots, n$ satisfaçam uma relação da forma

$$
|h(u)-h(v)| \leq c_{\eta}|u-v|\left(e^{\eta|u|^{N-1}}+e^{\eta|v|^{N-1}}\right), u, v \in \mathbb{R}^{n},
$$

3. $1<q<N$ e $f_{i}$ e $g_{i}, i=1,2, \cdots, n$ satisfaçam (5) com expoentes $\rho_{i}$ e $\overline{\rho_{i}}$, $i=1,2, \cdots, n$, respectivamente, tais que,

$$
\rho_{i} \leq \rho_{f}:=1+\frac{2 q}{N-q} \text { e } \overline{\rho_{i}} \leq \rho_{g}:=1+\frac{q}{N-q}, i=1,2, \cdots, n .
$$

Antes de enunciar o principal resultado do Capítulo 1 , observemos que estamos trabalhando com a seguinte definição de soluçāo para o problema (1):

Definição 1 [4] Diremos que uma função $x:[0, \tau] \rightarrow X^{1}$ é uma solução $\varepsilon$-regular do problema (1) se $x \in C\left([0, \tau], X^{1}\right) \cap C\left((0, \tau], X^{1+\varepsilon}\right)$, e satisfaz

$$
x(t)=e^{-A t} x_{0}+\int_{0}^{t} e^{-A(t-s)} \sum_{i=1}^{p} H_{i}(x(s)) d s .
$$

Com isso podemos mostrar o seguinte resultado, cuja prova pode ser encontrada em [5], veja também [4] para detalhes dessa prova. No Capítulo 1 faremos a prova desse teorema, com alguns detalhes, para facilitar a leitura deste trabalho. 
Teorema 1 Suponhamos que para $i=1,2, \cdots, n f_{i}$ e $g_{i}$ satisfaçam as condições de crescimento dadas em (C1). Então, para todo $u_{0} \in L^{q}\left(\Omega, \mathbb{R}^{n}\right)$, existe uma única solução local, $u\left(. ; u_{0}\right)$, do problema $(1)$, satisfazendo $u\left(0 ; u_{0}\right)=u_{0}$, e dependendo continuamente de $u_{0} \in L^{q}\left(\Omega, \mathbb{R}^{n}\right)$. Ainda, esta solução é clássica para todo $t>0$.

Se $i=1,2, \cdots, n$ e $f_{i}$ e $g_{i}$ satisfazem as condições de crescimento dadas em (C2). Então, para todo $u_{0} \in W^{1, q}\left(\Omega, \mathbb{R}^{n}\right)$, existe uma única solução local, $u\left(\cdot ; u_{0}\right)$, do proble$m a(1)$, satisfazendo $u\left(0 ; u_{0}\right)=u_{0}$, e dependendo continuamente de $u_{0} \in W^{1, q}\left(\Omega, \mathbb{R}^{n}\right)$. Ainda, esta solução é clássica para todo $t>0$.

No Capítulo 2 descreveremos um critério de comparação abstrato desenvolvido em [6]. Neste artigo este critério foi utilizado para obter comparação e positividade de soluções para problemas do tipo (3). Na Seção 2.1 adapateremos alguns resultados contidos em [12], os quais nos permitirão aplicar este critério para obtermos comparação e positividade de soluções para o problema (1), e mesmo para problemas mais gerais. Usando estes resultados de comparação e positividade, podemos mostrar a existência global das soluções do problema (1) encontradas no Teorema 1. Para isso, precisamos assumir as seguintes restrições sobre o sinal dos termos não lineares de (1): suponhamos que existam constantes $B_{0}, C_{0} \in \mathbb{R}$ e $B_{1}, C_{1} \geq 0$ tais que para todo $u=\left(u_{1}, \cdots, u_{n}\right) \in$ $\mathbb{R}^{n}$,

$$
\begin{aligned}
& u_{i} f_{i}(u) \leq-C_{0} u_{i}^{2}+C_{1}\left|u_{i}\right|, \\
& u_{i} g_{i}(u) \leq-B_{0} u_{i}^{2}+B_{1}\left|u_{i}\right|
\end{aligned}
$$

$i=1,2, \cdots, n$.

Ainda mais, com o mesmo raciocínio utilizado em [6] e em [20], mostraremos a existência de atratores globais, $\mathcal{A}_{X}$, para o problema (1) com $n=2$, onde $X=$ $W^{1, q}\left(\Omega, \mathbb{R}^{2}\right)$ ou $X=L^{q}\left(\Omega, \mathbb{R}^{2}\right)$, isto é feito assumindo que o primeiro autovalor, $\lambda_{1}$, do problema

$$
\left\{\begin{array}{l}
-\Delta u+C_{0} u=\lambda u \quad \text { em } \Omega, \\
\frac{\partial u}{\partial \vec{n}}+B_{0} u=0 \quad \text { em } \Gamma,
\end{array}\right.
$$

seja positivo, onde $B_{0}, C_{0}$ sāo dados em (6). Da mesma forma que em [6] e [20], obteremos também limites uniformes para pontos do atrator global. As condições (6) e (7) são as mesmas utilizadas em [5] para obter existência global e existência de atratores globais para o problema (3). Resumimos os principais resultados do Capítulo 2 no seguinte teorema: 
Teorema 2 Seja $X=L^{q}\left(\Omega, \mathbb{R}^{2}\right)$ ou $X=W^{1, q}\left(\Omega, \mathbb{R}^{2}\right)$, e suponhamos que para $i=$ $1,2, f_{i}, g_{i}: \mathbb{R} \rightarrow \mathbb{R}$ sejam funções satisfazendo (C1), (C2) e (6). Suponhamos também que o primeiro autovalor do problema (7) seja positivo. Então, o problema (1) tem um atrator compacto global $\mathcal{A}_{X}$ tal que

$$
\mathcal{A}_{X} \subset \Sigma\left(\phi_{1}, \phi_{2}\right):=\left\{u \in L^{\infty}\left(\Omega, \mathbb{R}^{2}\right) ;\left|u_{i}(x)\right| \leq \phi_{i}(x), x \in \Omega, i=1,2\right\},
$$

onde $\phi$ é a solução do seguinte problema elíptico:

$$
\begin{aligned}
& -\Delta \phi_{1}+C_{0} \phi_{1}=C_{1} \quad \text { em } \Omega, \\
& -\Delta \phi_{2}+C_{0} \phi_{2}=C_{1} \quad \text { em } \Omega, \\
& \frac{\partial \phi_{1}}{\partial \vec{n}}+B_{0} \phi_{1}+k\left(\phi_{1}-\phi_{2}\right)=B_{1} \quad \text { em } \Gamma, \\
& \frac{\partial \phi_{2}}{\partial \vec{n}}+B_{0} \phi_{2}-k\left(\phi_{1}-\phi_{2}\right)=B_{1} \text { em } \Gamma .
\end{aligned}
$$

Obtemos também no Captítulo 2, os mesmos resultados obtidos acima para o caso em que $k=0$ e $n \geq 1$ no problema (1), e um resultado de semicontinuidade superior desses atratores em relação ao parâmetro $\lambda$.

Considerando $n=2$ e $k>0$ em (1), suponhamos que as condições do Teorema 2 estejam satisfeitas e que existam constantes positivas $C_{f}$ e $C_{g}$ tais que

$$
\sup _{s \in \mathbb{R}}\left|f_{1}(s)-f_{2}(s)\right| \leq C_{f} \text { e } \sup _{s \in \mathbb{R}}\left|g_{1}(s)-g_{2}(s)\right| \leq C_{g} .
$$

Veremos que com os resultados obtidos no Capítulo 2, essa condição não é restritiva. O objetivo do Capítulo 3 é provarmos o seguinte teorema:

Teorema 3 Suponhamos que as funções $f_{i}, g_{i}: \mathbb{R} \rightarrow \mathbb{R}, i=1,2$ satisfaçam as condições acima. Então, existe uma constante $k_{0}>0$ tal que para todo $k>k_{0}$,

$$
\lim _{t \rightarrow \infty}\|u(t, .)-v(t, .)\|_{L^{\infty}(\Omega)} \leq C\left(\left|C_{f}\right|+\left|C_{g}\right|\right)
$$

onde $C_{f}$ e $C_{g}$ são dadas em (8). Ainda mais, se $f_{1}=f_{2}$ e $g_{1}=g_{2}$, então

$$
\lim _{t \rightarrow \infty}\|u(t, .)-v(t, .)\|_{L^{\infty}(\Omega)}=0
$$

para todo $k>k_{0}$. 
A propriedade (9) é chamada de "sincronização". Sincronização em problemas parabólicos foi considerado em [9] para problemas parabólicos com condição de fronteira do tipo Neumann, e com o acoplamento ocorrendo dentro da região $\Omega$. Neste trabalho obtemos sincronização em problemas parabólicos com condição de fronteira não linear, e o acoplamento ocorrendo na fronteira da região.

Tomando $k=0, n \geq 1$ e $g_{i} \equiv 0, i=1,2, \ldots, n$, em [14] foi mostrado que existe uma vizinhança, $\mathcal{N}\left(\mathcal{A}_{0}\right)$, de $\mathcal{A}_{0}$ em $\mathbb{R}^{n}$ tal que

$$
\mathcal{A}_{D} \subset \mathcal{N}\left(\mathcal{A}_{0}\right)
$$

para $d:=\min \left\{d_{1}, \cdots, d_{n}\right\}$ suficientemente grande, onde $\mathcal{A}_{D}$ é o atrator global compacto para o problema (1) com as restrições acima e $\mathcal{A}_{0}$ é o atrator global da equação diferencial ordinária limite:

$$
\dot{v}(t)=F(v(t))
$$

Tal propriedade é chamada de homogeneização. O mesmo resultado também foi obtido em [15], mas no caso em que as não linearidades $g_{i}, i=1,2, \cdots, n$, são lineares. No Capítulo 4 obtemos os mesmos resultados no caso em que $g_{i}, i=1,2, \cdots, n$, são funções não lineares não nulas, satisfazendo as condições dos Capítulos 1 e 2 , e a equação diferencial ordinária limite é substituida por

$$
\dot{v}(t)=F(v(t))+|\Gamma| G(v(t)) .
$$

Observemos também que no problema tratado em [14], o espaço das funções constantes já é uma variedade invariante para (1) com $k=0$ e $g_{i} \equiv 0, i=1,2, \cdots, n$, fato que não ocorre no caso em que $g_{i} \not \equiv 0, i=1,2, \cdots, n$. Isso mostra que o problema em questāo é relevante. 


\section{Capítulo 1}

\section{Unicidade e Existência Local de Soluções}

Seja $\Omega \subset \mathbb{R}^{N}$, um domínio aberto, limitado com fronteira $\Gamma:=\partial \Omega$ suave. Consideremos o problema

$$
\begin{cases}u_{t}(t, x)=D \Delta u(t, x)+F(u(t, x)) \quad & t>0, \quad x \in \Omega \\ D \frac{\partial u}{\partial \vec{n}}(t, x)=\lambda G(u(t, x)) \quad t>0, & x \in \Gamma\end{cases}
$$

onde

- $u=\left(u_{1}, u_{2}, \cdots, u_{n}\right)^{\top}, n \geq 1$

- $\frac{\partial u}{\partial \vec{n}}=\langle\nabla u, \vec{n}\rangle, \vec{n}$ é a normal à $\Gamma$, apontando para fora de $\Omega$;

- $D$ é a seguinte matriz $n \times n$ :

$$
D:=\left[\begin{array}{lllll}
d_{1} & 0 & 0 & \cdots & 0 \\
0 & d_{2} & 0 & \cdots & 0 \\
0 & 0 & d_{3} & \cdots & 0 \\
\vdots & \vdots & \vdots & \ddots & \vdots \\
0 & 0 & 0 & \cdots & d_{n}
\end{array}\right]
$$

com $d_{i}>0$, para todo $i=1,2, \cdots, n$; 
- $F=\left(F_{1}, F_{2}, \cdots, F_{n}\right): \mathbb{R}^{n} \rightarrow \mathbb{R}^{n}$ e $G=\left(G_{1}, G_{2}, \cdots, G_{n}\right): \mathbb{R}^{n} \rightarrow \mathbb{R}^{n}$ são duas funções tais que se $u=\left(u_{1}, u_{2}, \cdots, u_{n}\right)$, então

$$
\begin{aligned}
& F_{i}(u)=f_{i}(u), \quad i=1,2, \cdots, n, \\
& G_{1}(u)=g_{1}(u)-k\left(u_{1}-u_{2}\right), \\
& G_{2}(u)=g_{2}(u)+k\left(u_{1}-u_{2}\right), \\
& G_{i}(u)=g_{i}(u), \quad i=3,4, \cdots, n, \text { se } n>2,
\end{aligned}
$$

onde $f_{i}, g_{i}: \mathbb{R}^{n} \rightarrow \mathbb{R}, i=1,2, \cdots, n$, são funções localmente lipschitzianas;

- $k$ e $\lambda$ são constantes positivas.

O objetivo deste capítulo é mostrar que o problema (1.1) possui uma única solução, $u\left(t ; u_{0}\right)$, para todo dado inicial $u_{0} \in L^{q}\left(\Omega, \mathbb{R}^{n}\right)$, ou $u_{0} \in W^{1, q}\left(\Omega, \mathbb{R}^{n}\right)$ e que esta soluçāo depende continuamente do dado inicial $u_{0}$. Para fazermos isto, transformaremos o problema (1.1) em um problema abstrato da forma

$$
\begin{aligned}
& \dot{u}(t)=-A u(t)+\sum_{i=1}^{p} H_{i} u(t), t>0, \\
& u(0)=u_{0},
\end{aligned}
$$

onde $A$ é um operador setorial definido em um espaço de Banach $X$ e $H_{i}, i=1,2, \cdots, p$ são funções adequadas, as quais serão definidas posteriormente. Mostramos que o problema (1.3) é localmente bem posto em X, e finalmente aplicaremos estes resultados para mostrar que o problema (1.1) é localmente bem posto em $L^{q}\left(\Omega, \mathbb{R}^{n}\right)$ e em $W^{1, q}\left(\Omega, \mathbb{R}^{n}\right)$. Os resultados deste capítulo, podem ser encontradas, para $n=1$, com detalhes em [5]. Introduziremos aqui algumas notaçōes, as quais serão úteis no desenvolver deste trabalho. Consideremos $\left\{X^{\alpha}, \alpha \geq 0\right\}$, a escala de espaços de potências fracionárias associadas ao operador $A$. Estaremos sempre procurando soluções de (1.1) definidas como segue:

Definição 1.1 Diremos que uma função $x:[0, \tau] \rightarrow X^{1}$ é uma solução $\varepsilon$-regular do problema (1.1) se $x \in C\left([0, \tau], X^{1}\right) \cap C\left((0, \tau], X^{1+\varepsilon}\right)$, e satisfaz

$$
x(t)=e^{-A t} x_{0}+\int_{0}^{t} e^{-A(t-s)} \sum_{i=1}^{p} H_{i}(x(s)) d s .
$$

Com relação às não linearidades em (1.3), elas deverão satisfazer a seguinte condição de $\varepsilon$-regularidade: 
Definição 1.2 Para todo $\varepsilon \geq 0$, diremos que uma aplicação $f$ é $\varepsilon$-regular relativamente ao $\operatorname{par}\left(X^{1}, X^{0}\right)$, se existe $\rho>1$ e $\gamma(\varepsilon)$ satisfazendo $\rho \varepsilon \leq \gamma(\varepsilon)<1$ e uma constante $C$ tal que $f: X^{1+\varepsilon} \rightarrow X^{\gamma(\varepsilon)}$ e

$$
\|f(x)-f(y)\|_{X^{\gamma(\varepsilon)}} \leq C\|x-y\|_{X^{1+\varepsilon}}\left(\|x\|_{X^{1+\varepsilon}}^{\rho-1}+\|y\|_{X^{1+\varepsilon}}^{\rho-1}+1\right),
$$

para todo $x, y \in X^{1+\varepsilon}$.

Notemos que se $f: X^{1+\varepsilon} \rightarrow X^{\gamma(\varepsilon)}$ é uma aplicação satisfazendo (1.5), então

$$
\|f(x)\|_{X^{\gamma(\varepsilon)}} \leq C\left(\|x\|_{X^{1+\varepsilon}}^{\rho}+1\right)
$$

Este capítulo está organizado da seguinte forma: na Seção 1 construíremos uma escala de espaços de Banach, a qual será utilizada, na Seção 2, para mostrar que o problema (1.3) é localmente bem posto em $X$. Finalmente, na Seção 3 aplicamos os resultados obtidos nas seções anteriores para mostrar que o problema (1.1) é localmente bem posto $L^{q}\left(\Omega, \mathbb{R}^{n}\right)$ e em $W^{1, q}\left(\Omega, \mathbb{R}^{n}\right)$.

\subsection{Escalas de Espaços de Banach}

Nesta seção contruíremos uma escala de espaços de Banach associada a um operador sectorial $A: D(A) \subset E \rightarrow E$, densamente definido em um espaço de Banach $E$. Os resultados desta seção podem ser encontrados com mais detalhes em [3].

\subsubsection{Conceitos Gerais}

Começaremos primeiro com alguns conceitos gerais de interpolação, os quais passaremos a descrever agora.

Definição 1.3 Sejam $E_{0}, E_{1}$ espaços de Banach, o par $\left(E_{0}, E_{1}\right)$ será chamado um par de interpolação, se existe um espaço localmente convexo, $X$, tal que para $j=1,2$,

$$
E_{j} \hookrightarrow X
$$

isto é, $E_{j} \subset X, j=1,2$, com inclusão contínua. 
Observemos se $E_{1} \hookrightarrow E_{0}$, então podemos tomar $X=E_{0}$. Ao longo dessa subseção e das posteriores, para simplificar a notação e as contas, vamos assumir que os pares de interpolaçāo $\left(E_{0}, E_{1}\right)$ sejam tais que

$$
E_{1} \stackrel{d}{\hookrightarrow} E_{0},
$$

isto é, $E_{1} \subset E_{0}$, com inclusão contínua e $E_{1}$ é denso em $E_{0}$.

Definição $1.4 S e\left(E_{0}, E_{1}\right)$ é um par de interpolação satisfazendo (1.7) $e$

$$
E_{1} \stackrel{d}{\hookrightarrow} E \stackrel{d}{\hookrightarrow} E_{0},
$$

então E é chamado de espaço intermediário com relação à $\left(E_{0}, E_{1}\right)$.

Consideremos

$$
\mathcal{B}=\{E: \quad E \text { é um espaço de Banach }\}
$$

e

$$
\mathcal{B}_{1}=\left\{\left(E_{0}, E_{1}\right):\left(E_{0}, E_{1}\right) \text { é um par de interpolaçao satisfazendo (1.7) }\right\} .
$$

Os morfismos em $\mathcal{B}$ são os operadores lineares limitados, e a composição é a composição usual de aplicaçōes. Os morfismos em $\mathcal{B}_{1}$ são elementos, $A$, tais que para: $\left(E_{0}, E_{1}\right),\left(F_{0}, F_{1}\right) \in \mathcal{B}_{1}$ temos

$$
A \in \mathcal{L}\left(E_{0}, F_{0}\right) \cap \mathcal{L}\left(E_{1}, F_{1}\right),
$$

onde a composição é a composição usual de aplicações. Denotaremos tal morfismo por $A:\left(E_{0}, E_{1}\right) \rightarrow\left(F_{0}, F_{1}\right)$.

Definição 1.5 Consideremos $\left(E_{0}, E_{1}\right) e\left(F_{0}, F_{1}\right)$ pares de interpolação satisfazendo (1.7). Chamaremos $E$ e $F$ de espaços de interpolação, se $E$ e $F$ são espaços intermediários com respeito a $\left(E_{0}, E_{1}\right)$ e $\left(F_{0}, F_{1}\right)$, respectivamente, e se $A:\left(E_{0}, E_{1}\right) \rightarrow\left(F_{0}, F_{1}\right)$, então $A \in \mathcal{L}(E, F)$.

Ainda mais, $E$ e $F$ são chamados de espaços de interpolação com expoente $\theta \in$ $(0,1)$, com respeito a $\left(E_{0}, E_{1}\right)$ e $\left(F_{0}, F_{1}\right)$, se existir $c(\theta) \in \mathbb{R}^{+}$tal que

$$
\|A\|_{\mathcal{L}(E, F)} \leq c(\theta)\|A\|_{\mathcal{L}\left(E_{0}, F_{0}\right)}^{1-\theta}\|A\|_{\mathcal{L}\left(E_{1}, F_{1}\right)}^{\theta},
$$

onde $A:\left(E_{0}, E_{1}\right) \rightarrow\left(F_{0}, F_{1}\right)$. Se além disso, $c(\theta)=1$, então $E$ e $F$ são chamados de espaços exatos de interpolação com expoente $\theta \in(0,1)$, com respeito a $\left(E_{0}, E_{1}\right) e$ $\left(F_{0}, F_{1}\right)$. 
Definiçāo 1.6 Um functor (exato) de interpolação de expoente $\theta \in(0,1)$, denotado por $(\cdot, \cdot)_{\theta}$, é uma aplição de $\mathcal{B}_{1}$ em $\mathcal{B}$ tal que para $\left(E_{0}, E_{1}\right),\left(F_{0}, F_{1}\right) \in \mathcal{B}_{1}, E_{\theta}:=$ $\left(E_{0}, E_{1}\right)_{\theta}$ e $F_{\theta}:=\left(F_{0}, F_{1}\right)_{\theta}$ são espaços (exatos) de interpolação com expoente $\theta \in(0,1)$. Ainda mais, se $A:\left(E_{0}, E_{1}\right) \rightarrow\left(F_{0}, F_{1}\right)$, então $A \in \mathcal{L}\left(\left(E_{0}, E_{1}\right)_{\theta},\left(F_{0}, F_{1}\right)_{\theta}\right)$ e

$$
\|A\|_{\mathcal{L} E_{\theta}, F_{\theta}} \leq c(\theta)\|A\|_{\mathcal{L}\left(E_{0}, F_{0}\right)}^{1-\theta}\|A\|_{\mathcal{L}\left(E_{1}, F_{1}\right)}^{\theta} \quad(c(\theta)=1) .
$$

Daremos agora um exemplo de functor de interpolação de expoente $\theta \in(0,1)$, que será fundamental para o desenvolvimento deste trabalho, para maiores detalhes $\mathrm{e}$ propriedades sobre esse functor veja [7].

Exemplo 1.1 Seja $\left(E_{0}, E_{1}\right)$ um par de interpolação tal que $E_{1} \stackrel{d}{\rightarrow} E_{0}$. Definamos o espaço $\mathcal{F}\left(\left(E_{0}, E_{1}\right)\right)$, como sendo o conjunto de todas as funções $f: \mathbb{C} \rightarrow E_{0}$, que são limitadas e contínuas na faixa

$$
S:=\{z \in \mathbb{C}: 0 \leq \operatorname{Re} z \leq 1\},
$$

são analíticas na faixa aberta

$$
S_{0}:=\{z \in \mathbb{C}: 0<\operatorname{Re} z<1\}
$$

$e$, além disso, as funções $t \longmapsto f(j+i t), j=0,1$, são contínuas da reta real em $E_{j}$, $j=0,1$ e satisfazem

$$
\lim _{|t| \rightarrow \infty} f(j+i t)=0, \quad j=0,1 .
$$

$O$ espaço $\mathcal{F}\left(\left(E_{0}, E_{1}\right)\right)$, munido da norma

$$
\|f\|_{\mathcal{F}}:=\max \left\{\sup _{t \in \mathbb{R}}\|f(i t)\|_{E_{0}}, \sup _{t \in \mathbb{R}}\|f(1+i t)\|_{E_{1}}\right\}
$$

é um espaço de Banach.

Definamos, para todo $\theta \in(0,1)$, o espaço

$$
E_{[\theta]}:=\left[E_{0}, E_{1}\right]_{\theta}:=\left\{a \in E_{0}: a=f(\theta), \text { para alguma } f \in \mathcal{F}\left(\left(E_{0}, E_{1}\right)\right)\right\} .
$$

Consideremos uma norma em $E_{[\theta]}$ dada por

$$
\|a\|_{[\theta]}:=\inf \left\{\|f\|_{\mathcal{F}}: f(\theta)=a, f \in \mathcal{F}\right\} .
$$

Então $E_{[\theta]}$ é um espaço intermediário exato de interpolação com expoente $\theta \in(0,1)$ $e[\cdot, \cdot]_{\theta}$ é um functor exato de interpolação com expoente $\theta$, o qual é denominado functor de interpolação complexo de expoente $\theta \in(0,1)$. 
Vamos agora introduzir o conceito de escalas de espaços de Banach. Sejam $B$ : $D(B) \subset G \rightarrow G$ um operador linear, $F$ e $G$ espaços de Banach tais que $F \hookrightarrow G$. Denotaremos por $B_{F}$, a $F$-realização de $B$ em $F$, isto é

$$
\begin{aligned}
& D\left(B_{F}\right)=\{x \in D(B) \cap F: B x \in F\} \\
& B_{F} x=B x, \forall x \in D\left(B_{F}\right) .
\end{aligned}
$$

Para facilitar a notação, daqui para frente, consideremos

$$
\operatorname{Lis}(X, Y):=\left\{T \in \mathcal{L}(X, Y) ; T \text { é bijetor e } T^{-1} \in \mathcal{L}(Y, X)\right\}
$$

onde $X, Y$ são espaços de Banach. Temos assim a:

Definição 1.7 Denotemos por $\Lambda$ o conjunto dos números naturais, ou o conjunto dos números inteiros, ou o conjunto do números reais maiores ou iguais a zero, ou o conjunto dos números reais. Para cada $\alpha \in \Lambda$, sejam $E_{\alpha}:=\left(E_{\alpha},\|\cdot\|_{\alpha}\right)$ um espaço de Banach e $A_{\alpha} \in \operatorname{Lis}\left(E_{\alpha+1}, E_{\alpha}\right.$.) Se para $\alpha, \beta \in \Lambda, \alpha>\beta$, tivermos

$$
E_{\alpha} \hookrightarrow E_{\beta}
$$

e o diagrama

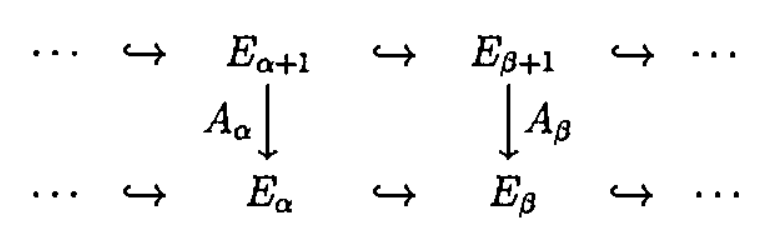

for comutativo, então

$$
\left\{\left(E_{\alpha}, A_{\alpha}\right): \alpha \in \Lambda\right\}
$$

será chamada uma escala de espaços de Banach sobre o conjunto de índices $\Lambda$. A escala será dita unilateral, se $\Lambda=\mathbb{N}$, ou $\Lambda=\mathbb{R}^{+}$, caso contrário ela será chamada bilateral. A escala será chamada discreta se $\Lambda=\mathbb{N}$, ou $\Lambda=\mathbb{Z}$, caso contrário ela será chamada contínua. A escala de espaços de Banach $\left[\left(E_{\alpha}, A_{\alpha}\right): \alpha \in \Lambda\right]$ é densamente imersa se $E_{\alpha} \stackrel{d}{\rightarrow} E_{\beta}$, para quaisquer $\alpha, \beta \in \Lambda$, tais que $\alpha>\beta$.

Definiçāo 1.8 Diremos que duas escalas de Banach $\left\{\left(E_{\alpha}, A_{\alpha}\right) ; \alpha \in \Lambda\right\}$ e $\left\{\left(F_{\alpha}, B_{\alpha}\right)\right.$; $\alpha \in \Lambda\}$ sobre o mesmo conjunto de índices $\Lambda$ são isomorfas se existir $I_{\alpha} \in \operatorname{Lis}\left(E_{\alpha}, F_{\alpha}\right)$, $\alpha \in \Lambda$, tal que dados $\alpha, \beta \in \Lambda, \alpha>\beta$, então $E_{\alpha} \hookrightarrow E_{\beta}, F_{\alpha} \hookrightarrow F_{\beta}$ e o diagrama 


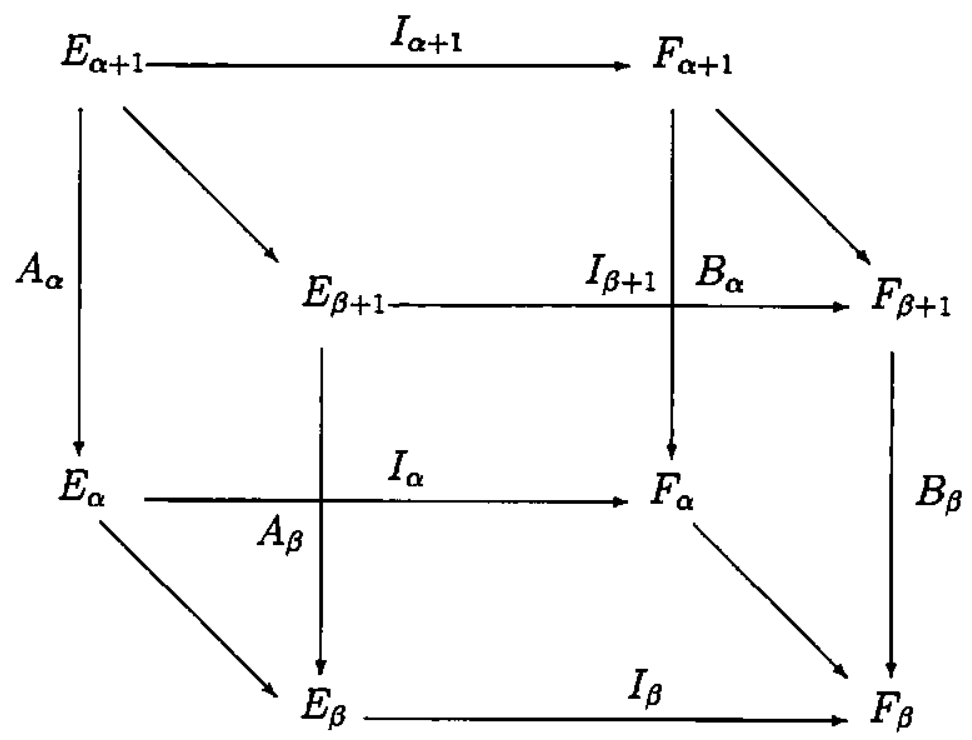

for comutativo. Se para cada $\alpha \in \Lambda, I_{\alpha}$ for uma isometria, diremos que as duas escalas são isométricamente isomorfas. As duas escalas de Banach são equivalentes se elas são isomorfas e se $E_{\alpha}=F_{\alpha}$ para $\alpha \in \Lambda$, ou seja, se os espaços são os mesmos com normas equivalentes.

Finalmente temos a:

Definição 1.9 Uma escala de Banach $\left\{\left(E_{\alpha}, A_{\alpha}\right) ; \alpha \in \Lambda\right\}$ sobre um conjunto de índices $\Lambda$ é dita ser reflexiva, se cada $E_{\alpha}$ é um espaço de Banach reflexivo. A escala é dita ser uma escala de Hilbert, se cada $E_{\alpha}$ é um espaço de Hilbert. Diremos que a escala de Banach satisfaz as desigualdades de interpolação se

$$
\|e\|_{\beta} \leq c(\alpha, \beta, \gamma)\|e\|_{\gamma}^{(\alpha-\beta) /(\alpha-\gamma)}\|e\|_{\alpha}^{(\beta-\gamma) /(\alpha-\gamma)}, \quad e \in E_{\alpha},
$$

para $\alpha, \beta, \gamma \in \Lambda$ com $\gamma<\beta<\alpha$.

\subsubsection{Escalas de Potência}

Nesta subseção exibiremos uma técnica simples para construir uma escala unilateral discreta de espaços de Banach. Sejam $E:=(E,\|\cdot\|)$ um espaço de Banach e $A: D(A) \subset$ $E \rightarrow E$ um operador linear fechado, densamente definido tal que $0 \in \rho(-A)$. Neste caso, sabemos que para todo $j=0,1, \cdots, A^{j}: D\left(A^{j}\right) \subset E \rightarrow E$ é um operador linear fechado, onde $A^{0}=I_{E}$, e $0 \in \rho\left(A^{j}\right)$. 
Definamos

$$
E_{j}:=\left(E_{j},\|\cdot\|_{j}\right):=E_{j}(A):=\left(D\left(A^{j}\right),\left\|A^{j} \cdot\right\|_{E}\right), \quad j=0,1,2, \cdots,
$$

onde $E_{0}:=E:=(E,\|\cdot\|)$. Como $0 \in \rho\left(A^{j}\right)$, então $\|\cdot\|_{j}$ é equivalente a norma do gráfico de $A^{j}$. Como $A^{j}$ é um operador fechado, $E_{j}$ é um espaço de Banach. Ainda, temos que

$$
\|e\|_{k} \leq\left\|A^{k-j}\right\|_{L(E)}\|e\|_{j}, \quad e \in E_{j}, \quad j>k,
$$

e isto implica que $E_{j} \hookrightarrow E_{k}$ se $j>k$. Portanto, $A_{j}:=E_{j}$-realização de $A$ está bem definida. Temos assim o

Teorema 1.1 Sejam $E$ um espaço de Banach e $A: D(A) \subset E \rightarrow E$ um operador linear fechado tal que $0 \in \rho(A)$. Definamos $E_{j}$ e $A_{j}$ para $j=0,1,2, \cdots$ como acima. Então

$$
\left\{\left(E_{j}, A_{j}\right) ; j=0,1,2, \cdots\right\}
$$

é uma escala de Banach unilateral discreta densamente imersa, a qual chamaremos de escala de potências gerada por $(E, A)$.

Demonstração: A primeira parte segue da construção de $E_{j}, A_{j}, j=0,1, \cdots$. Agora, como $A$ é densamente definido, então $E_{1} \stackrel{d}{\hookrightarrow} E$. Logo, para todo $e_{0} \in E_{j}, j=1,2, \cdots$, como

$$
A^{-j} \in \operatorname{Lis}\left(E, E_{j}\right) \cap \operatorname{Lis}\left(E_{1}, E_{j+1}\right)
$$

para todo $j=0,1, \cdots$, então $A^{j} e_{0} \in E_{0}$ e, portanto, existe uma sequência $e_{n} \in E_{1}$, $n \geq 1$, tal que

$$
\left\|e_{n}-A^{j} e_{0}\right\|_{E_{0}} \rightarrow 0, \text { quando } n \rightarrow \infty
$$

Assim,

$$
\left\|A^{-j} e_{n}-e_{0}\right\|_{E_{j}}=\left\|A^{-j} e_{n}-A^{-j} A^{j} e_{0}\right\|_{E_{j}} \leq c\left\|e_{n}-A^{j} e_{0}\right\|_{E_{0}}
$$

$\operatorname{com} A^{-j} e_{n} \in E_{j+1}$, o que implica que

$$
E_{j+1} \stackrel{d}{\hookrightarrow} E_{j}
$$


para $j \geq 1$. Assim, para todo $x \in E_{k}$, e todo $\varepsilon>0$, existe $x_{k+1} \in E_{k+1}$ tal que

$$
\left\|x_{k+1}-x\right\|_{E_{k}} \leq \frac{\varepsilon}{2}
$$

Da mesma forma, existe $x_{k+2} \in E_{k+2}$ tal que

$$
\left\|x_{k+2}-x_{k+1}\right\|_{E_{k+1}} \leq \frac{\varepsilon}{\left\|A^{-1}\right\|_{L(E)}} .
$$

Juntado esses dois fatos, segue que

$$
\left\|x_{k+2}-x\right\|_{E_{k}} \leq \varepsilon
$$

o que mostra que $E_{k+2} \stackrel{d}{\rightarrow} E_{k}$, para todo $k \geq 1$, procedendo um número finito de vezes prova-se que

$$
E_{j} \stackrel{d}{\rightarrow} E_{k}
$$

para todo $j \geq k$, o que concluí a prova do teorema.

O próximo resultado será útil para as próximas subseções.

Proposição 1.1 Seja $m$ um inteiro positivo e definamos $\left(F_{j}, B_{j}\right):=\left(E_{j+m}, A_{j+m}\right)$ para $j=0,1,2, \cdots$. Então $\left\{\left(F_{j}, B_{j}\right) ; j=0,1,2, \cdots\right\}$ é a escala de potência gerada por $\left(E_{m}, A_{m}\right)$. Ela é isometricamente isomorfa a escala de potências gerada por $(E, A)$.

Demonstração: Notemos inicialmente que $A_{j+m}$ é a $E_{m}$-realização de $A_{m}$ para todo $j=0,1, \cdots$. Também, $A_{m}: D\left(A_{m}\right) \subset E_{m} \rightarrow E_{m}$ é um operador linear fechado e $0 \in \rho\left(A_{m}\right)$, para todo inteiro $m$, o que implica a primeira parte da afirmação.

Agora, para cada $j=0,1, \cdots$, definamos

$$
I_{j}:=\left(A_{j}\right)^{-m} \text {. }
$$

Como

$$
\left(A_{j}\right)^{-m} \in L i s\left(E_{j}, E_{m+j}\right), j=0,1,2, \cdots,
$$

temos para todo $e \in E_{j}$, que

$$
\left\|\left(A_{j}\right)^{-m} e\right\|_{E_{m+j}}=\left\|A^{m}\left(A_{j}\right)^{-m} e\right\|_{E_{j}}=\|e\|_{E_{j}},
$$

da mesma forma obtemos, para $e \in E_{j+m}$, que

$$
\left\|\left(A_{j}\right)^{-m} e\right\|_{E_{j}}=\|e\|_{E_{j+m}},
$$

o que mostra que $I_{j}$ é um isomorfismo isométrico, o resultado segue agora da Definição 1.8 . 


\subsubsection{Espaços de Extrapolação}

Seja $E$ um espaço vetorial normado. Um completamento $(\tilde{E}, \tilde{j})$ de $E$, consiste de um espaço de Banach, $\tilde{E}$, e de um isomorfismo isométrico, $\tilde{j}$, de $E$ sobre um subespaço denso de $\tilde{E}$.

Um completamento particular pode ser obtido tomando como sendo $\tilde{E}$, o espaço de Banach cujos elementos as são classes de equivalência, $\widetilde{\left(e_{j}\right)}$, das sequências de Cauchy em $E$, onde duas sequências de Cauchy, $\widetilde{\left(e_{j}\right)}$ e $\widetilde{\left(f_{j}\right)}$, são equivalentes se

$$
\lim _{j \rightarrow \infty}\left\|e_{j}-f_{j}\right\|_{E}=0
$$

Este espaço é dotado de uma estrutura linear natural, e sua norma é definida por

$$
\left\|\widetilde{\left(e_{j}\right)}\right\|_{\bar{E}}:=\lim _{j \rightarrow \infty}\left\|e_{j}\right\|_{E}
$$

Então $\tilde{j}(e):=\widetilde{(e)}$, onde $(e)$ denota a sequência $(e, e, e, \cdots)$ para cada $e \in E$, é um isomorfismo isométrico sobre a imagem de $E$ em $\tilde{E}$, a qual é densa em $\tilde{E}$.

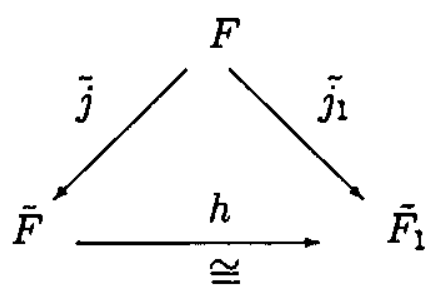

Se $\left(\tilde{E}_{1}, \tilde{j}_{1}\right)$ é um outro completamento de $E$, então existe uma isometria $h \in$ $\operatorname{Lis}\left(\tilde{E}, \tilde{E}_{1}\right)$ tal que o diagrama acima é comutativo. Neste sentido completamentos são únicos, exceto por uma isometria. Logo, fixaremos um completamento particular $(\tilde{E}, \tilde{j})$ de $E$, e identificaremos $E$ com o subespaço $\tilde{j}(E)$ de $\tilde{E}$.

Ao longo desta subseção, $E=\left(E,\|\cdot\|_{E}\right)$ é um espaço de Banach e $A: D(A) \subset E \rightarrow E$ é um operador linear fechado e densamente definido tal que $0 \in \rho(-A)$. Consideremos $\left\{\left(E_{j}, A_{j}\right) ; j=0,1,2, \cdots\right\}$ a escala de potências unilateral discreta gerada por $(E, A)$.

Observemos que $\left\|A^{-1} \cdot\right\|_{E}$ é uma norma em $E$, mais fraca que $\|\cdot\|_{E}$. Portanto, o completamento

$$
\left(E_{-1},\|\cdot\|_{-1}\right):=E_{-1}(A):=\left(E,\left\|A^{-1} \cdot\right\|_{E}\right)^{\sim}
$$


do espaço vetorial normado $\left(E,\left\|A^{-1} \cdot\right\|_{E}\right)$ é um espaço de Banach bem definido e

$$
E \hookrightarrow E_{-1}
$$

Este espaço é chamado, espaço de extrapolação de $E$ gerado por $A$. Observemos que

$$
\|A e\|_{-1}=\|e\|_{E}, \quad e \in E_{1}
$$

e que

$$
\left\|A^{-1} f\right\|_{E}=\|f\|_{-1}, \quad f \in E .
$$

Então segue de (1.11) e (1.12) que existe uma única extensāo contínua $A_{-1}$ de $A$ tal que $A_{-1}$ é um isomorfismo isométrico de $E_{0}:=E$ sobre $E_{-1}$ e $\left(A_{-1}\right)^{-1} \subset A^{-1}$. Diremos que $A_{-1}$ é a $E_{-1}$-realização de $A$. Para provar o próximo resultado, precisamos do seguinte lema, cuja prova pode ser encontrada em [3], página 250.

Lema 1.1 Sejam $X$ e $Y$ espaços de Banach com $Y \hookrightarrow X$ e seja $B: D(B) \subset X \rightarrow X$, um operador linear fechado. Suponhamos que $(\mu+B)^{-1}(Y) \subset Y$ para algum $\mu \in$ $\rho(-B)$. Então $\mu \in \rho\left(-B_{Y}\right) e$

$$
\left(\mu+B_{Y}\right)^{-1}=\left.(\mu+B)^{-1}\right|_{Y}
$$

Se $\operatorname{dom}(B) \subset Y$ então $\rho(B)=\rho\left(B_{Y}\right)$ e (1.13) vale para cada $\mu \in \rho(-B)$.

É fácil ver que $D(B) \subset Y$ se, e somente se, $(\mu+B)^{-1}(X) \subset Y$ para algum $\mu \epsilon$ $\rho(-B)$. Iterando a equação resolvente, podemos generalizar o Lema 1.1, para o caso em que

$$
(\mu+B)^{-k}(X) \subset Y
$$

para algum inteiro positivo $k$.

Proposição 1.2 Com as notações acima temos

(i) $A_{-1} \in \operatorname{Lis}\left(E_{0}, E_{-1}\right) \cap \mathcal{C}\left(E_{-1}\right)$ e é um isomorfismo isométrico;

(ii) A é fechável em $E_{-1}$ e seu fecho é igual a $A_{-1}$;

(iii) $\rho\left(A_{-1}\right)=\rho(A)$. 
Demonstração: Como $A_{-1} \in \operatorname{Lis}\left(E_{0}, E_{-1}\right)$, a norma do gráfico de $A_{-1}$ é uma norma equivalente para $E_{0}$. Portanto, $A_{-1}: E_{0} \subset E_{-1} \rightarrow E_{-1}$ é um operador linear fechado, o que mostra (i). A prova de (ii) agora é imediata. Os itens (i) e (ii), e o Lema 1.1, provam o item (iii).

Como $A_{-1}: D\left(A_{-1}\right) \subset E_{-1} \rightarrow E_{-1}$ é um operador linear fechado tal que $0 \in \rho\left(A_{-1}\right)$ podemos definir, como acima, $E_{-2}:=E_{-1}\left(A_{-1}\right)$, o espaço de extrapolação de $E_{-1}$ gerado por $A_{-1}$, e tal que $A_{-2}$, a $E_{-2}$-realização de $A_{-1}$, esteja bem definida. Assim, obtemos que

$$
E \stackrel{d}{\rightarrow} E_{-1} \stackrel{d}{\rightarrow} E_{-2}
$$

que $A_{-2}: E_{-2} \subset E_{-1} \rightarrow E_{-1}$ é um operador fechado tal que

$$
A_{-2} \in \operatorname{Lis}\left(E_{-1}, E_{-2}\right) \cap \mathcal{C}\left(E_{-2}\right),
$$

e é um isomorfismo isométrico e que $A_{-1}$ é fechável em $E_{-2}$ com fecho $A_{-2}$.

Observemos que estas relações devem ser interpretadas com cuidado. Mais precisamente, sejam respectivamente $\left(E_{-1}, j_{-1}\right)$ e $\left(E_{-2}, j_{-2}\right)$ completamentos de $\left(E,\left\|A^{-1}\right\|_{E}\right)$ e $\left(E_{-1},\left\|\left(A_{-1}\right)^{-1}\right\|_{E_{-1}}\right)$. Entāo

$$
E \stackrel{j_{-1}}{\longrightarrow} E_{-1} \stackrel{j_{-2}}{\longrightarrow} E_{-2}
$$

e $j_{-1}$ e $j_{-2}$ são isometrias lineares sobre subespaços de $E_{-1}$ e $E_{-2}$, respectivamente. Portanto, se identificamos como acima $E_{-1}$ com sua imagem $j_{-2}\left(E_{-1}\right)$ em $E_{-2}$ também identificamos $j_{-1}(E)$ com $j_{-2}\left(j_{-1}(E)\right.$ ). Portanto na construção de $E_{-2}$ temos que substituir o espaço de extrapolação já construído $E_{-1}$ por uma imagem isomorfa, de tal forma que (1.14) seja válida.

Teorema 1.2 Suponhamos que $A: D(A) \subset E \rightarrow E$ seja um operador fechado, densamente definido tal que $0 \in \rho(A)$. Fixemos $m=0,1,2, \cdots$ e consideremos $E_{-k}$ e $A_{-k}$ para $1 \leq k \leq m$, definidos indutivamente por

$$
E_{-k}:=\left(E_{-k},\|\cdot\|_{-k}\right):=\left(E_{-k-1},\left\|\left(A_{-k+1}\right)^{-1} \cdot\right\|_{E}\right)^{\sim}
$$

$e$

$$
A_{-k}:=\text { fecho de } A_{-k+1} \text { em } E_{-k} .
$$


Definamos

$$
\left(F_{j}, B_{j}\right):=\left(E_{j-m}, A_{j-m}\right), \quad j=0,1,2, \cdots .
$$

Então $\left\{\left(F_{j}, B_{j}\right) ; j=0,1,2, \cdots\right\}$ é a escala de potência gerada por $\left(E_{-m}, A_{-m}\right)$. Ela é isometricamente isomorfa a escala de potências gerada por $(E, A)$.

Demonstração: Pelo visto acima, $\left(E_{-k}, A_{-k}\right)$ estão bem definidos para $1 \leq k \leq m$. Logo, (1.11)-(1.12) implicam que $\left\{\left(F_{j}, B_{j}\right) ; j=0,1,2, \cdots\right\}$ é a escala de potências gerada por $\left(E_{-m}, A_{-m}\right)$. A última parte do teorema segue agora da Proposição 1.1 .

Definição 1.10 Com as notações acima, sejam $A$ um operador densamente definido e $m$ um inteiro positivo. Então $\left\{\left(E_{j-m}, A_{j-m}\right) ; j=0,1,2, \cdots\right\}$ é chamada escala extrapolada de potências de ordem $m$, gerada por $(E, A)$ e denotaremos por

$$
\left\{\left(E_{j}, A_{j}\right) ; j=-m,-m+1,-m+2, \cdots\right\}
$$

Observemos que pelo Teorema 1.1, a escala acima é uma escala de Banach densamente imersa, unilateral e discreta.

\subsubsection{Escalas Duais}

Ao longo desta seção assumiremos que $E$ é um espaço de Banach reflexivo e que $A: D(A) \subset E \rightarrow E$ é um operador linear fechado, densamente definido tal que $0 \in$ $\rho(-A)$, a menos que se afirme o contrário. Recordemos que $A^{\prime}: D\left(A^{\prime}\right) \subset E^{\prime} \rightarrow E^{\prime}$ é um operador linear fechado, densamente definido e $\rho\left(A^{\prime}\right)=\rho(A)$. Para evitar confusāo consideremos

$$
E^{\#}:=E^{\prime}, \quad A^{\#}:=A^{\prime}
$$

O objetivo principal desta subseção é mostrar o seguinte teorema de dualidade:

Teorema 1.3 Sejam $E$ um espaço de Banach reflexivo e $A: D(A) \subset E \rightarrow E$ um operador linear fechado, densamente definido tal que $0 \in \rho(A)$. Então as escalas de potências bilaterais densamente imersas

$$
\left\{\left(E_{k}, A_{k}\right) ; k=0, \pm 1, \pm 2, \cdots\right\}
$$


$e$

$$
\left\{\left(E_{k}^{\#}, A_{k}^{\#}\right) ; k=0, \pm 1, \pm 2, \cdots\right\}
$$

geradas por $(E, A)$ e $\left(E^{\#}, A^{\#}\right)$, onde $E^{\#}:=E^{\prime}$ e $A^{\#}:=A^{\prime}$ respectivamente, são escalas de Banach reflexivas, bem definidas e densamente imersas. Ainda mais,

$$
\left(E_{k}\right)^{\prime}=E_{-k}^{\#}, \quad\left(A_{k}\right)^{\prime}=A_{-k}^{\#}, \quad k=0, \pm 1, \pm 2, \cdots,
$$

relativamente ao par de dualidade induzido naturalmente por $E, E^{\prime}$.

A escala de potências (1.16) é chamada escala dual da escala de potências (1.15). Inicialmente vamos construir as escalas de potências enunciadas no Teorema 1.3. Para isso, precisamos considerar as escalas de potências extrapoladas densamente imersas de ordem $m, m=1,2,3, \cdots$,

$$
\left\{\left(E_{j}, A_{j}\right) ; \quad j=-m,-m+1,-m+2, \cdots\right\}
$$

e

$$
\left\{\left(E_{j}^{\#}, A_{j}^{\#}\right) ; \quad j=-m,-m+1,-m+2, \cdots\right\}
$$

geradas por $(E, A)$ e $\left(E^{\#}, A^{\#}\right)$ respectivamente, estão bem definidas. Vamos utilizar essas escalas para construirmos as escalas no Teorema 1.3, e mostrarmos as propriedades afirmadas no referido teorema. Denotemos por $(\cdot, \cdot\rangle:=\langle\cdot, \cdot\rangle_{E^{\prime}, E}$ o par de dualidade entre $E^{\prime}$ e $E$. Temos o:

Lema 1.2 Seja $m$ um inteiro positivo, para todo inteiro $k$ tal que $1 \leq k \leq m$, consideremos

$$
\left\langle\mathrm{e}^{\#}, e\right\rangle_{k}=\left\langle\left(A_{-k}^{\#}\right)^{-k} \mathrm{e}^{\#}, A^{k} e\right\rangle, \quad e \in E_{k}, \quad \mathrm{e}^{\#} \in E_{-k}^{\#} .
$$

Então

$$
\langle\cdot, \cdot\rangle_{k}: E_{-k}^{\#} \times E_{k} \rightarrow \mathbb{K}
$$

é uma forma bilinear contínua de norma 1.

Lema 1.3 Sejam $E$ e $F$ espaços vetoriais e $B$ e $C$ operadores lineares com dominios em $E$ e imagem em $F$ tal que $B \supset C$. Se $B$ é injetor e $C$ é sobrejetor, então $B=C$. 
Denotemos por $\left(A_{k}\right)^{\prime}$ o dual do operador linear fechado densamente definido, $A_{k}$, como um operador linear em $E_{k}$ e não como um operador linear contínuo de $E_{k+1} \mathrm{em}$ $E_{k}$. Temos assim o seguinte resultado de dualidade:

Proposição 1.3 Sejam $m$ um inteiro positivo e $k$ um inteiro tal que $1 \leq k \leq m$. Então com as notações acima temos

$$
\left(E_{k}\right)^{\prime}=E_{-k}^{\#}
$$

$e$

$$
\left(A_{k}\right)^{\prime}=A_{-k}^{\#}
$$

relativamente ao par de dualidade $\langle\cdot, \cdot\rangle_{k}$.

Demonstração: O Lema 1.2 implica que

$$
\mathrm{e}^{\#} \rightarrow\left\langle\mathrm{e}^{\#}, \cdot\right\rangle_{k}
$$

define uma isometria linear entre $E_{-k}^{\#}$ e $\left(E_{k}\right)^{\prime}$.

Para todo $f \in\left(E_{k}\right)^{\prime}$, como $A^{k} \in \operatorname{Lis}\left(E_{k}, E\right)$, existe $f^{\#} \in E^{\#}$ tal que o diagrama

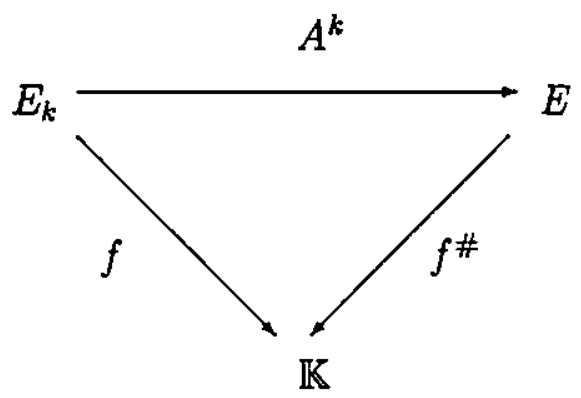

é comutativo, isto é,

$$
f(e)=\left\langle f^{\#}, A^{k} e\right\rangle, \quad e \in E_{k} .
$$

Também, como $\left(A_{-k}^{\#}\right)^{k} \in \operatorname{Lis}\left(E^{\#}, E_{-k}^{\#},\right)$ então o elemento

$$
\mathrm{e}^{\#}:=\left(A_{-k}^{\#}\right)^{k} f^{\#} \in E_{-k}^{\#}
$$


está bem definido. Logo, (1.21) juntamente com o Lema 1.2 implicam que

$$
f(e)=\left\langle\mathrm{e}^{\#}, e\right\rangle_{k}, \text { para } e \in E_{k} .
$$

Portanto $\left(E_{k}\right)^{\prime}=E_{-k}^{\#}$.

Agora, como $D\left(A_{k}\right)=E_{k+1}$ é denso em $E_{k}$, o dual $\left(A_{k}\right)^{\prime}$ de $A_{k}$ relativamente ao par de dualidade $\langle\cdot, \cdot\rangle_{k}$ está bem definido e (1.19) implica que $\left(A_{k}\right)^{\prime}: D\left(\left(A_{k}\right)^{\prime}\right) \subset E_{k}^{\#} \rightarrow E_{k}^{\#}$ é um operador linear fechado. Também, $A_{k}$ é a $E_{k}$-realização de $A$ e $A_{-k}^{\#} \supset A^{\#}$, logo obtemos que

$$
\begin{aligned}
\left\langle\mathrm{e}^{\#}, A_{k} e\right\rangle_{k} & =\left\langle\left(A_{-k}^{\#}\right)^{-k} \mathrm{e}^{\#}, A A^{k} e\right\rangle=\left\langle A^{\#}\left(A_{-k}^{\#}\right)^{-k} \mathrm{e}^{\#}, A^{k} e\right\rangle \\
& =\left\langle\left(A_{-k}^{\#}\right)^{-k} A_{-k}^{\#} \mathrm{e}^{\#}, A^{k} e\right\rangle=\left\langle A_{-k}^{\#} \mathrm{e}^{\#}, e\right\rangle_{k}
\end{aligned}
$$

para $\mathrm{e}^{\#} \in E_{-k+1}^{\#}$ e para todo $e \in E_{k+1}$. Portanto,

$$
\left(A_{k}\right)^{\prime} \supset A_{-k}^{\#} \text {. }
$$

Mas $\rho\left(A_{k}\right)=\rho\left(\left(A_{k}\right)^{\prime}\right)$, e o Teorema 1.2 implica que

$$
0 \in \rho\left(\left(A_{k}\right)^{\prime}\right) \cap \rho\left(A_{-k}^{\#}\right) \text {. }
$$

Daí, o Lema 1.3 mostra que

$$
\left(A_{k}\right)^{\prime}=A_{-k}^{\#}
$$

concluíndo a prova da proposição.

Corolário 1.1 Se $0 \leq \ell<k \leq m$,

$$
\left\langle\mathrm{e}^{\#}, e\right\rangle_{k}=\left\langle\mathrm{e}^{\#}, e\right\rangle_{\ell}, \quad e \in E_{k}, \quad \mathrm{e}^{\#} \in E_{-\ell}^{\#} .
$$

Uma consequência do corolário acima é que

$$
\left\langle\mathrm{e}^{\#}, e\right\rangle_{k}=\left\langle\mathrm{e}^{\#}, e\right\rangle, \quad e \in E_{k}, \quad \mathrm{e}^{\#} \in E^{\#}, \quad 0<k \leq m .
$$

Desde que $E^{\#}$ é denso em $E_{-k}^{\#}$ vemos que $\langle\cdot, \cdot\rangle_{k}$ é unicamente determinado por $\langle\cdot, \cdot\rangle \mathrm{e}$ que $\langle\cdot, \cdot\rangle_{k}$ é naturalmente induzida por $\langle\cdot, \cdot\rangle$. Daqui por diante, abusando da notação, para todo inteiro positivo $m$ e $k, 0 \leq k \leq m$ escreveremos $\langle\cdot, \cdot\rangle$ para denotar $\langle\cdot, \cdot\rangle_{k}$, se não houver possibilidade de confusão.

Como consequência imediata da Proposição 1.3 podemos provar o próximo teorema, e a principal caracterização dos espaços de extrapolação $E_{-k}$ para $1 \leq k \leq m$. 
Teorema 1.4 Para todo inteiro positivo $m$ e $k$ tal que $k \geq-m, E_{k}$ e $E_{k}^{\#}$ são espaços de Banach reflexivos. Ainda mais, relativamente ao par de dualidade $\langle\cdot, \cdot\rangle$, temos que

$$
\left(E_{k}\right)^{\prime}=E_{-k}^{\#}, \quad\left(A_{k}\right)^{\prime}=A_{-k}^{\#}, \quad-m \leq k \leq m .
$$

Demonstração: Como $E_{k}$ [respectivamente $E_{k}^{\#}$ ] é isomorfo a $E$ [respectivamente $E^{\#}$ ] e como $E$, portanto $E^{\#}$, é reflexivo, a primeira afirmativa segue. Da Proposição 1.3 sabemos que (1.22) é válida para $0 \leq k \leq m$. Portanto

$$
E_{k}=\left(E_{-k}^{\#}\right)^{\prime}, \quad A_{k}=\left(A_{-k}^{\#}\right)^{\prime}, \quad 0 \leq k \leq m
$$

relativamente ao par de dualidade $\langle\cdot, \cdot\rangle$.

Para todo $k \in[-m, \infty)$, consideremos

$$
\left(F_{k}, B_{k}\right):=\left(E_{k}^{\#}, A_{k}^{\#}\right)
$$

Então (1.22) com $k=0$ implica que a escala dual $\left\{\left(F_{k}, B_{k}\right) ; k \geq-m\right\}$ é igual a escala (1.17). Portanto, aplicando novamente (1.22) para $F_{k}$ e $B_{k}$, obtemos que

$$
E_{k}^{\#}=\left(E_{-k}\right)^{\prime} \quad \text { e } \quad A_{k}^{\#}=\left(A_{-k}\right)^{\prime}
$$

relativamente ao par de dualidade $\langle$,$\rangle , provando o teorema.$

Corolário 1.2 Se $1 \leq k \leq m$ então $E_{-k}=\left(E_{k}^{\#}\right)^{\prime}$ e $A_{-k}=\left(A_{k}^{\#}\right)^{\prime}$ relativamente ao par de dualidade $\langle\cdot, \cdot\rangle$.

Consideremos

$$
\left\{\left(E_{j}, A_{j}\right) ; j=0,1,2, \cdots\right\}
$$

e

$$
\left\{\left(E_{j}^{\#}, A_{j}^{\#}\right) ; j=0,1,2, \cdots\right\}
$$

as escalas de potências unilaterais associadas a $(E, A)$ e $\left(E^{\#}, A^{\#}\right)$, respectivamente. Temos

$$
E_{j} \stackrel{d}{\rightarrow} E_{k} \stackrel{d}{\rightarrow} E
$$


para $0<k<j$, então

$$
\begin{aligned}
& E^{\prime} \stackrel{d}{\hookrightarrow} E_{k}^{\prime} \stackrel{d}{\hookrightarrow} E_{j}^{\prime}, \\
& \left\langle x^{\prime}, x\right\rangle_{E_{j}, E_{j}^{\prime}}=\left\langle x^{\prime}, x\right\rangle_{E_{k}, E_{k}^{\prime}}=\left\langle x, x^{\prime}\right\rangle_{E, E^{\prime}},
\end{aligned}
$$

para todo $x^{\prime} \in E^{\prime}, x \in E$, e $0<k<j$. Logo o Teorema 1.4 implica que

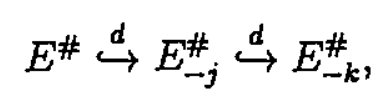

para $0 \leq k \leq j \leq m$, para todo inteiro positivo $m$. Trocando $m$ por $\tilde{m}$, a cadeia de inclusões acima ainda continua válida, sem alterar a cadeia obtida para $m$. Obtemos assim, a seguinte escala de potências bilaterais densamente imersa

$$
\left\{\left(E_{j}^{\#}, A_{j}^{\#}\right) ; j=0, \pm 1, \pm 2, \cdots\right\}
$$

gerada por $\left(E^{\#}, A^{\#}\right)$.

Da mesma forma, começando co a escala (1.24) e usando o Corolário 1.1, obtemos a seguinte escala de potências bilaterais densamente imersa

$$
\left\{\left(E_{j}, A_{j}\right) ; j=0, \pm 1, \pm 2, \cdots\right\}
$$

gerada por $(E, A)$.

As propriedades afirmadas no Teorema 1.3 seguem agora do Teorema 1.4.

\subsubsection{Escalas de Interpolação-Extrapolação}

Sejam $E$ um espaço de Banach e $A: D(A) \subset E \rightarrow E$ um operador linear fechado, densamente definido tal que $0 \in \rho(A)$. Então, dado $m=0,1,2, \cdots$, a escala de potências extrapolada discreta

$$
\left\{\left(E_{j}, A_{j}\right) ; j=-m,-m+1,-m+2, \cdots\right\}
$$

de ordem $m$ gerada por $(E, A)$, é uma escala de Banach densamente imersa bem definida. Escolhamos para cada $\theta \in(0,1)$ o funtor de interpolação complexo de expoente $\theta \in(0,1),[\because \cdot]_{\theta}$, como definido no Exemplo 1.1, e consideremos

$$
E_{\alpha}:=\left[E_{j}, E_{j+1}\right]_{\alpha-j}, \quad A_{\alpha}:=E_{\alpha} \text {-realização de } A_{j}
$$

para $\alpha \in(j . j+1)$ e $j=-m,-m+1,-m+2, \cdots$. Temos então o seguinte teorema: 
Teorema 1.5 Sobre as condições acima, $\left\{\left(E_{\alpha}, A_{\alpha}\right) ; \alpha \geq-m\right\}$ é uma escala de Banach densamente imersa, denominada escala de interpolação-extrapolação de ordem $m$ gerada por $(E, A)$ e $[\cdot, \cdot]_{\theta}, 0<\theta<1$.

Suponhamos agora que $E$ é um espaço de Banach reflexivo e consideremos o functor de interpolação dual, $(\cdot, \cdot)_{\theta}^{\#}$, como sendo

$$
(\cdot, \cdot)_{\theta}^{\#}:=[\cdot, \cdot]_{\theta} .
$$

Portanto, a escala dual de interpolaçāo-extrapolação

$$
\left\{\left(E_{\alpha}^{\#}, A_{\alpha}^{\#}\right) ; \alpha \in \mathbb{R}\right\},
$$

isto é, a escala de intepolação-extrapolação gerada por $\left(E^{\#}, A^{\#}\right)$ e $(\cdot, \cdot)_{\theta}^{\#}, 0<\theta<1$, é uma escala de Banach densamente imersa bem definida.

Teorema 1.6 Suponhamos que as condições acima estjem satisfeitas. Então para todo $\alpha \in \mathbb{R},\left(E_{\alpha}\right)^{\prime}=E_{-\alpha}^{\#}$ e $\left(A_{\alpha}\right)^{\prime}=A_{-\alpha}^{\#}$, relativamente ao par de dualidade induzido naturalmente pelo par de dualidade, $\langle\cdot, \cdot\rangle$, entre $E$ e $E^{\prime}$. Ainda, $E_{\alpha}$ é reflexivo para todo $\alpha \in \mathbb{R}$.

Demonstraçāo: A prova deste teorema segue de propriedades do functor de interpolação complexo, e dos resultados da Subseção 1.1.4.

\subsubsection{Semigrupos em Escalas de Interpolação-Extrapolação}

Nesta subseção estudaremos o comportamento de semigrupos em escalas interpolação-extrapolação. Os resultados desta subseção serão extremamente úteis na prova de existência e unicidade de soluções do problema (1.1), bem como propriedades de regularização de tais soluções.

Antes de prosseguirmos, precisamos introduzir algumas notações. Dados $M \geq 1 \mathrm{e}$ $\sigma \in \mathbb{R}$, denotaremos por

$$
\mathcal{G}(E, M, \sigma)
$$

o conjunto de todos os operadores lineares fechados $A: D(A) \subset E \rightarrow E$ tais que $-A$ são geradores infinitesimais de semigrupos fortemente contínuos, $\left\{e^{-t A} ; t \geq 0\right\}$, em $E$ e satisfazem

$$
\left\|e^{-t A}\right\|_{\mathcal{L}(E)} \leq M e^{\sigma t}, \quad t \geq 0
$$


Definimos por $\mathcal{G}(E)$ o conjunto de todos os geradores de semigrupos fortemente contínuos em $E$. Ainda, se $A \in \mathcal{G}(E)$, definamos

$$
\operatorname{tipo}(-A):=\inf \{\sigma \in \mathbb{R}: \exists M \geq 1 \text { tal que } A \in \mathcal{G}(E, M, \sigma)\} .
$$

Podemos agora enunciar o principal resultado deste capítulo. Sejam $E$ um espaço de Banach, $B: D(B) \subset E \rightarrow E$ um operador linear fechado, densamente definido tal que $0 \in \rho(-B), m=0,1,2, \cdots$ e $(\cdot, \cdot)_{\theta}, 0<\theta<1$, functores exatos de interpolação de expoente $\theta$.

Denotemos por

$$
\left\{\left(E_{\alpha}, B_{\alpha}\right) ; \alpha \geq-m\right\}
$$

a escala de interpolação-extrapolação de ordem $m$ gerada por $(E, B)$ e $(\cdot, \cdot)_{\theta}, 0<\theta<1$ e por

$$
\left\{\left(E_{j}^{\#}, B_{j}^{\#}\right) ; j=0,1,2, \cdots\right\}
$$

a escala de potências dual gerada por $\left(E^{\#}, B^{\#}\right):=\left(E^{\prime}, B^{\prime}\right)$.

Dado $A: D(A) \subset E \rightarrow E$ um outro operador linear fechado, densamente definido definamos $A^{\#}:=A^{\prime}$ e denotemos por $A_{\alpha}$ a $E_{\alpha}$-realização de $A$ para $\alpha \in \mathbb{R}$ que é bem definido. Ainda, se $A$ é fechável em $E_{-\alpha}$ para algum $\alpha \in(0, m]$, então podemos considerar

$$
A_{-\alpha}:=\text { fecho de } A \text { em } E_{-\alpha}
$$

e denotemos por $A_{-\alpha}$ a $E_{-\alpha}-$ realização de $A$. Notemos que $A_{\alpha}: D\left(A_{\alpha}\right) \subset E_{\alpha} \rightarrow E_{\alpha}$ é um operador fechado sempre que ele estiver bem definido.

Seja $n=0,1,2, \cdots$ e suponhamos que $\rho(A) \neq \emptyset \mathrm{e}$

$$
D\left(A^{k}\right)=E_{k}, \quad 0 \leq k \leq n, \quad k=0,1,2, \cdots .
$$

Então, dado $\mu \in \rho(-A)$ como $D\left((\mu+A)^{k}\right)=D\left(A^{k}\right)$ para $k=0,1,2, \cdots$, segue do Teorema do Gráfico Fechado que $(\mu+A)^{k} B^{-k}$ e $B^{k}(\mu+A)^{-k}$ pertencem à $\mathcal{L}(E)$ para $0 \leq k \leq n$, ou equivalentemente, que

$$
(\mu+A)^{k} \in \mathcal{L}\left(E_{k}, E\right), \quad(\mu+A)^{-k} \in \mathcal{L}\left(E, E_{k}\right)
$$


para $0 \leq k \leq n$. Semelhantemente, se

$$
D\left(\left(A^{\#}\right)^{k}\right)=E_{k}^{\#}, \quad 0 \leq k \leq m,
$$

como $\rho(A)=\rho\left(A^{\#},\right)$ obtemos que

$$
\left(\mu+A^{\#}\right) \in \mathcal{L}\left(E_{k}^{\#}, E^{\#}\right), \quad\left(\mu+A^{\#}\right)^{-k} \in \mathcal{L}\left(E^{\#}, E_{k}^{\#}\right)
$$

para $0 \leq k \leq m$. Assim,

$$
\begin{aligned}
N(A, \mu, m, n):= & \text { máximo das normas de todos operadores } \\
& \text { ocorrendo em (1.26) e (1.28) }
\end{aligned}
$$

é bem definido se (1.25) e (1.27) estão satisfeitas.

Sejam $E_{0} \stackrel{d}{\leftrightarrow} E_{1}$ espaços de Banach, denotaremos por $H\left(E_{0}, E_{1}\right)$, o conjunto de todos os operadores $A \in \mathcal{L}\left(E_{0}, E_{1}\right)$ tais que $-A$, considerado como um operador linear em $E_{1}$ com domínio $E_{0}$, é gerador infinitesimal de um semigrupo fortemente contínuo analítico em $\mathcal{L}\left(E_{1}\right)$. Dados $k \geq 1$ e $\omega>0$, escrevemos $A \in H\left(E_{0}, E_{1}, k, \omega\right)$ se $\omega+A \epsilon$ $\operatorname{Lis}\left(E_{0}, E_{1}\right)$ e

$$
k^{-1} \leq \frac{\|(\lambda+A) x\|_{E_{1}}}{|\lambda|\|x\|_{E_{1}}+\|x\|_{E_{1}}} \leq k
$$

para todo $x \in E_{0}$, e todo $\operatorname{Re} \lambda \geq \omega$.

Finalmente temos o

Teorema 1.7 Sejam $E$ um espaço de Banach, $B: D(B) \subset E \rightarrow E$ um operador linear fechado, densamente definido tal que $0 \in \rho(B), m=0,1,2, \cdots e(\cdot, \cdot)_{\theta}, 0<\theta<1$, functores exatos de interpolação de expoente $\theta$. Consideremos $\left\{\left(E_{\alpha}, B_{\alpha}\right) ; \alpha \geq-m\right\}$ a escala de interpolação de ordem $m$ gerada por $(E, B)$ e $(\cdot, \cdot)_{\theta}, 0<\theta<1$, e seja $\left\{\left(E_{j}^{\#}, B_{j}^{\#}\right) ; j=0,1,2, \cdots\right\}$ a escala de potências dual.

Suponhamos que $A \in \mathcal{G}(E)$, e que exista $n=0,1,2, \cdots$ tais que

$$
D\left(A^{k}\right)=E_{k}, \quad 0 \leq k \leq n
$$

$e$

$$
D\left(\left(A^{\#}\right)^{k}\right)=E_{k}^{\#}, \quad 0 \leq k \leq m
$$


Então $A_{\alpha} \in \mathcal{G}\left(E_{\alpha}\right)$ e $\rho\left(A_{\alpha}\right)=\rho(A)$ para $-m \leq \alpha \leq n$. Ainda mais, os diagramas

$$
\begin{array}{ccc}
E_{\alpha} & \stackrel{e^{-t A_{a}}}{\longrightarrow} & E_{\alpha} \\
\downarrow & & \downarrow \\
E_{\beta} & \stackrel{\mathrm{e}^{-t A_{\beta}}}{\longrightarrow} & E_{\beta}
\end{array}
$$

são comutativos para $-m \leq \beta \leq \alpha \leq n$ e para todo $t \geq 0$.

Mais precisamente, suponhamos que $A \in \mathcal{G}(E, M, \omega)$ para algum $M \geq 1$ e $\omega \in \mathbb{R}$. Fixemos $\mu \in \rho(-A)$ e definamos $N:=N(A, \mu, m, n)$. Então

$$
A_{\alpha} \in \mathcal{G}\left(E_{\alpha}, N^{2} M, \omega\right), \quad-m \leq \alpha \leq n
$$

e se $n \geq 1$,

$$
\lambda+A_{\alpha} \in \operatorname{Lis}\left(E_{\alpha+1}, E_{\alpha}\right), \quad \lambda \in \rho(-A), \quad-m \leq \alpha \leq n-1 .
$$

Ainda mais,

$$
\left\|A_{\alpha}\right\|_{\mathcal{L}\left(E_{\alpha+1}, E_{\alpha}\right)} \leq N^{2}\|A\|_{\mathcal{L}\left(E_{1}, E\right)}
$$

$e$

$$
\left\|\left(\lambda+A_{\alpha}\right)^{-1}\right\|_{\mathcal{L}\left(E_{\alpha}, E_{\alpha+j}\right)} \leq N^{2}\left\|(\lambda+A)^{-1}\right\|_{\mathcal{L}\left(E, E_{j}\right)}
$$

para $\lambda \in \rho(-A), j=0,1, e-m \leq \alpha \leq n-1$.

Adicionalmente, se $A \in H\left(E_{1}, E_{0}\right), n \geq 1$ então $A_{\alpha} \in H\left(E_{\alpha+1}, E_{\alpha}\right)$ para $-m \leq$ $\alpha \leq n-1$. Na realidade temos que, se $A \in H\left(E_{1}, E_{0}, k, \omega_{0}\right)$ para algum $k \geq 1$ e $\omega_{0}>0$, então

$$
A_{\alpha} \in H\left(E_{\alpha+1}, E_{\alpha}, k_{0}, \omega_{0}\right), \quad-m \leq \alpha \leq n-1,
$$

onde $k_{0}:=N^{2}\left(1+k+k N^{2}\|A\|_{\mathcal{L}\left(E_{1}, E_{0}\right)}\right)$. Dado $\sigma>\operatorname{tipo}(-A)$,

$$
\left\|\mathrm{e}^{-t A_{\beta}}\right\|_{\mathcal{L}\left(E_{\beta}, E_{\alpha}\right)} \leq c t^{\beta-\alpha} \mathrm{e}^{\sigma t}, \cdot t>0,
$$

para $-m \leq \beta \leq \alpha \leq n$, onde $c$ depende somente de $\alpha-\beta$. 


\subsubsection{Um Exemplo}

Nesta subseção construíremos uma escala de Banach para um operador específico, a qual será utilizada ao longo deste trabalho.

Consideremos o operador $A=\operatorname{diag}\left(A_{1}, A_{2}, \cdots, A_{n}\right)$, onde para cada $i=1,2, \cdots, n$, $A_{i}$ é definido da seguinte maneira: sejam $A_{i}: D\left(A_{i}\right) \subset L^{q}(\Omega) \rightarrow L^{q}(\Omega)$, com

$$
\begin{aligned}
& D\left(A_{i}\right)=\left\{\phi \in W^{2, q}(\Omega) ; \frac{\partial \phi}{\partial \vec{n}}=0, \text { em } \Gamma\right\}:=W_{\mathcal{N}}^{2, q}(\Omega) ; \\
& A_{i} \phi=-d_{i} \Delta \phi .
\end{aligned}
$$

Sejam $\left(E_{i}\right)_{q}^{1}:=D\left(A_{i}\right), i=1,2, \cdots, n$ e $E:=(E)_{q}^{0}:=L^{q}(\Omega), 1<q<\infty$. Tomando $[\cdot, \cdot]_{\theta}$ como sendo o functor de interpolação complexo de expoente $\theta, A_{i}, i=1,2, \cdots, n$ possui uma escala de espaços de Banach, que para $0 \leq \alpha \leq 1$ satisfazem

$$
\left(E_{i}\right)_{q}^{\alpha}=\left[\left(E_{i}\right)_{q}^{1},\left(E_{i}\right)_{q}^{0}\right]_{\alpha}, \quad 1<q<\infty
$$

Definamos, para $\alpha \in[0,1], 1<q<\infty$,

$$
E_{q}^{\alpha}:=\left(E_{1}\right)_{q}^{\alpha} \times\left(E_{2}\right)_{q}^{\alpha} \times \cdots \times\left(E_{n}\right)_{q}^{\alpha} .
$$

Temos que, para $\alpha>\beta$ e $1<q<\infty$ a inclusão $E_{q}^{\alpha} \subset E_{q}^{\beta}$ é compacta, $E_{q}^{\frac{1}{2}}=$ $W^{1, q}\left(\Omega, \mathbb{R}^{n}\right)$ e obtemos de [2] que

$$
E_{q}^{\alpha}:=\left[W_{\mathcal{N}}^{2, q}\left(\Omega, \mathbb{R}^{n}\right), L^{q}\left(\Omega, \mathbb{R}^{n}\right)\right]_{\alpha} \hookrightarrow H_{q}^{2 \alpha}\left(\Omega, \mathbb{R}^{n}\right)
$$

onde $\alpha \in(0,1), W_{\mathcal{N}}^{2, q}\left(\Omega, \mathbb{R}^{n}\right)=\prod_{i=1}^{n} W_{\mathcal{N}}^{2, q}(\Omega)$ e $H_{q}^{2 \alpha}\left(\Omega, \mathbb{R}^{n}\right)$ são os espaços de Bessel, para detalhes veja $[2,7]$.

Para a escala com expoentes negativos, consideremos para $\theta \in(0,1)$, o functor de interpolação complexo de expoente $\theta$,

$$
(\cdot, \cdot)_{\theta}^{\#}:=[\cdot, \cdot]_{\theta}
$$

Portanto, obtemos a escala

$$
\left\{\left(E_{\alpha}^{\#}, A_{\alpha}^{\#}\right) ; \alpha \in \mathbb{R}\right\}
$$

e para todo $\alpha>0$ teremos

$$
E_{q}^{-\alpha}=\left(E_{\#}^{\alpha}\right)^{\prime} \text { e } A_{-\alpha}=\left(A_{\alpha}^{\#}\right)
$$


Com as notações acima, resumiremos agora os resultados das subseções anteriores, os quais nos serão mais úteis para o desenvolvimento deste trabalho.

Teorema 1.8 Com as notações acima, se para todo $\alpha \in \mathbb{R} e 1<q<\infty$, $A_{\alpha}$ denotam a $E_{q}^{\alpha}$-realização de $A$ em $E$. Então $A_{\alpha} \in \mathcal{L}\left(E_{q}^{\alpha}, E_{q}^{\alpha}\right), 1<q<\infty e-A_{\alpha}: E_{q}^{\alpha+1} \subset$ $E_{q}^{\alpha} \rightarrow E_{q}^{\alpha}$ gera um semigrupo analítico $\left\{e^{-A_{\alpha} t} ; t \geq 0\right\}$ em $E_{q}^{\alpha}$. Existe uma constante $M \geq 1$ tal que

$$
t^{\beta-\alpha}\left\|e^{-A_{\alpha} t} e\right\|_{E_{q}^{\beta}} \leq M\|e\|_{E_{q}^{\alpha}}, \quad \alpha \leq \beta,
$$

a constante $M$ depende apenas de $\alpha$ e $\beta$.

\subsection{Existência Local de Soluções - Resultados Abs- tratos}

Nessa subseção mostraremos existência e unicidade de soluçōes para problemas da forma:

$$
\begin{aligned}
& \dot{x}(t)=-A x(t)+\sum_{i=1}^{p} H_{i} x(t), t>0, \\
& x(0)=x_{0} \in X,
\end{aligned}
$$

onde $A: D(A) \subset X \rightarrow X$ é um operador setorial definido em no espaço de Banach $X$, tal que $\operatorname{Re}(\sigma(A))>0$, onde $\sigma(A)$ denota o espectro do operador $A$. Os resultados desta seção estão todos contidos em [5], colocaremos esses resultados aqui para facilitar a leitura deste trabalho.

Consideremos $\left\{X^{\alpha}, \alpha \geq 0\right\}$, uma escala de espaços de Banach, como construída na Seção 1.1. Assumiremos que as não linearidades em (1.37) sejam elementos do conjunto $\mathcal{F}:=\mathcal{F}(\varepsilon, \rho, \gamma(\varepsilon), C), i=1,2, \cdots p$, onde $\mathcal{F}$ é definido como segue. Dado $0 \leq \varepsilon<1$, existem $\rho>1, \gamma(\varepsilon)>0$ com $\rho \varepsilon \leq \gamma(\varepsilon)<1$, e $C$ uma constante positiva, definimos $\mathcal{F}:=\mathcal{F}(\varepsilon, \rho, \gamma(\varepsilon), C)$ como sendo a família de funções $f$ tal que $f(\cdot)$ é uma aplicação $\varepsilon$-regular em relação ao par $\left(X^{1}, X^{0}\right)$, isto é, $f: X^{1+\varepsilon} \rightarrow X^{\gamma(\varepsilon)}$ satisfazendo

$$
\|f(x)-f(y)\|_{X^{\gamma(\varepsilon)}} \leq C\|x-y\|_{X^{1+\varepsilon}}\left(\|x\|_{X^{1+\varepsilon}}^{\rho-1}+\|y\|_{X^{1+\varepsilon}}^{\rho-1}+1\right)
$$

e

$$
\|f(x)\|_{X^{\gamma(c)}} \leq C\left(\|x\|_{X^{1+\varepsilon}}^{\rho}+1\right)
$$


para todo $x, y \in X^{1+\varepsilon}$. Conforme a Definição 1.1, estaremos procurando por soluções que satisfazem

$$
x(t)=e^{-A t} x_{0}+\int_{0}^{t} e^{-A(t-s)} \sum_{i=1}^{p} H_{i}(x(s)) d s .
$$

O objetivo desta seção é mostrarmos o seguinte teorema:

Teorema 1.9 Suponhamos que para $1 \leq i \leq p, H_{i} \in \mathcal{F}\left(\varepsilon_{i}, \rho_{i}, \gamma_{i}\left(\varepsilon_{i}, c\right)\right.$, para algum $\varepsilon_{i}>0$. Suponhamos também que

$$
\min \left\{\gamma_{i}\left(\varepsilon_{i}\right) ; 1 \leq i \leq p\right\}=: \underline{\gamma}>\max \left\{\varepsilon_{i} ; 1 \leq i \leq p\right\}=: \bar{\varepsilon} .
$$

Então, se $y_{0} \in X^{1}$, existe $r=r\left(y_{0}\right)>0$ e $\tau_{0}=\tau_{0}\left(y_{0}\right)>0$ tal que para todo $x_{0} \in X^{1}$, com

$$
\left\|x_{0}-y_{0}\right\|_{X^{1}}<r
$$

existe uma função contínua $x:\left[0, \tau_{0}\right] \rightarrow X^{1}$, com $x(0)=x_{0}$, que é uma solução $\bar{\varepsilon}$-regular fraca para (1.37) começando em $x_{0}$. Adicionalmente, esta solução satisfaz

$$
x \in C\left(\left(0, \tau_{0}\right], X^{1+\theta}\right), t^{\theta}\left\|x\left(t ; x_{0}\right)\right\|_{X^{1+\theta} \rightarrow 0}
$$

quando $t \rightarrow 0^{+}$, para todo $0<\theta<\underline{\gamma}$. Ainda, se $x_{0}, z_{0} \in B_{X^{1}}\left(y_{0}, r\right)$ temos

$$
t^{\theta} \| x\left(t ; x_{0}-x\left(t ; z_{0}\right)\left\|_{X^{1+\theta}} \leq C\left(\theta_{0}, \tau_{0}\right)\right\| x_{0}-z_{0} \|_{X^{1}}, \forall t \in\left[0, \tau_{0}\right]\right.
$$

para todo $0 \leq \theta \leq \theta_{0}<\underline{\gamma}$.

Ainda mais,

$$
x \in C\left(\left(0, \tau_{0}\right], X^{1+\underline{\gamma}}\right) \quad e \quad x_{t} \in C\left(\left(0, \tau_{0}\right], X^{1+\theta}\right)
$$

para todo $\theta<\underline{\gamma}$.

Dividiremos esta seção em duas subseções, na primeira mostraremos a existência local de soluções e na segunda mostraremos a unicidade de tais soluçōes. 


\subsubsection{Existência de Soluções}

Introduziremos primeiro algumas definições e demonstraremos alguns lemas técnicos. Suponhamos que para $i=1,2, \cdots, p$ as não linearidades $H_{i} \in \mathcal{F}\left(\varepsilon_{i}, \rho_{i}, \gamma_{i}\left(\varepsilon_{i}\right), C\right)$, para algum $\varepsilon_{i} \in[0,1)$.

Consideremos a função beta $B(\cdot, \cdot):(0, \infty) \times(0, \infty) \rightarrow(0, \infty)$, definida por

$$
B(a, b)=\int_{0}^{1}(1-x)^{a-1} x^{b-1} d x .
$$

Para $i=1,2, \cdots, p$, definamos

$$
B_{\varepsilon_{i}}^{\theta}:=\max _{0 \leq \xi \leq \theta}\left\{B\left(\gamma_{i}\left(\varepsilon_{i}\right)-\xi, 1-\rho_{i} \varepsilon_{i}\right), B\left(\gamma_{i}\left(\varepsilon_{i}\right)-\xi, 1-\gamma_{i}\left(\varepsilon_{i}\right)\right)\right\} .
$$

Escolhamos agora $r=r(\mu, M)$ tal que

$$
r=\frac{\mu}{4 M}
$$

Ainda, para $y_{0} \in X^{1}$ fixado, escolhamos $\delta>0$ e $\tau_{0}=\tau_{0}\left(y_{0}, A, \mu, \varepsilon_{i}, \rho_{i}, \gamma_{i}\left(\varepsilon_{\mathfrak{i}}\right), c, M\right)$ $\tau_{0} \in(0,1]$ tal que

$$
\sup _{1 \leq i, j \leq p} C M \delta B_{\varepsilon_{i}}^{\varepsilon_{j}}=\frac{\mu}{4 p},\left\|t^{\varepsilon_{i}} e^{-A t} y_{0}\right\|_{X^{1+\epsilon_{i}}} \leq \frac{\mu}{4} \text { e } \sup _{1 \leq i, j \leq p} \sup _{0 \leq t \leq \tau_{0}} t^{\gamma_{i}\left(\varepsilon_{i}\right)-\varepsilon_{j}} \leq \delta,
$$

Notemos que a segunda desigualdade é garantida pelo próximo lema, cuja prova pode ser encontrada com detalhes em [4].

Lema 1.4 Os operadores $t^{\alpha} e^{-A t}: X^{1} \rightarrow X^{1+\alpha}, t>0$ são operadores lineares limitados, satisfazendo

$$
\left\|t^{\alpha} e^{-A t}\right\|_{\mathcal{L}\left(X^{1}, X^{1+\alpha}\right)} \leq M,
$$

com constante $M$ independente de t. Ainda, para todo subconjunto compacto $J \subset X^{1}$ temos

$$
\limsup _{t \rightarrow 0}\left\|t_{x \in J}^{\alpha} e^{-A t} x\right\|_{X^{1+\alpha}}=0 .
$$

Para todo $x_{0} \in X^{1}$ com $\left\|x_{0}, y_{0}\right\|_{X^{1}}<r$, estaremos procurando por pontos fixos da aplicação

$$
(T x)(t)=e^{-A t} x_{0}+\int_{0}^{t} e^{-A(t-s)} \sum_{i=1}^{p} H_{i}(x(s)) d s
$$


no espaço

$$
K\left(\tau_{0}\right):=\left\{x \in C\left(\left(0, \tau_{0}\right], X^{1+\bar{\varepsilon}}\right) \mid \sup _{1 \leq i \leq p} \sup _{t \in\left(0, \tau_{0}\right]} t^{\varepsilon_{i}}\|x(t)\|_{X^{1+\varepsilon_{i}}} \leq \mu\right\}
$$

munido com a norma

$$
\|x\|_{K\left(\tau_{0}\right)}:=\sup _{1 \leq i \leq p} \sup _{t \in\left(0, \tau_{0}\right]} t^{\varepsilon_{i}}\|x(t)\|_{X^{1+e_{i}}}
$$

A idéia é usar o Princípio da Contração de Banach. Mostremos inicialmente algumas propriedades da aplicação $T$ definida acima. Temos o

Lema 1.5 Sejam $T$ e $K\left(\tau_{0}\right)$ como definidos acima. Com as escolhas dos parâmetros acima, então

$$
T\left(K\left(\tau_{0}\right)\right) \subset K\left(\tau_{0}\right)
$$

Demonstração: Tomemos $x \in K\left(\tau_{0}\right)$. Para cada $i=1,2, \cdots, p$, temos

$$
\begin{aligned}
& t^{\varepsilon_{i}}\|T x(t)\|_{X^{1+\varepsilon_{i}}} \leq\left\|t^{\varepsilon_{i}} e^{-A t} x_{0}\right\|_{X^{1+\varepsilon_{i}}}+\left\|t^{\varepsilon_{i}} \int_{0}^{t} e^{-A(t-s)} \sum_{j=1}^{p} H_{j}(x(s)) d s\right\|_{X^{1+\epsilon_{i}}} \\
& \leq\left\|t^{\varepsilon_{i}} e^{-A t} x_{0}\right\|_{X^{1+\varepsilon_{i}}}+M C t^{\varepsilon_{i}} \int_{0}^{t} \sum_{j=1}^{p}\left[(t-s)^{\gamma_{j}\left(\varepsilon_{j}\right)-\left(1+\varepsilon_{i}\right)}\right] d s \\
& +M C t^{\varepsilon_{i}} \int_{0}^{t} \sum_{j=1}^{p}\left[(t-s)^{\gamma_{j}\left(\varepsilon_{j}\right)-\left(1+\varepsilon_{i}\right)}\|x(s)\|_{X^{1+\varepsilon_{j}}}^{\rho_{j}}\right] d s \\
& \leq\left\|t^{\varepsilon_{i}} e^{-A t} x_{0}\right\|_{X^{1+\varepsilon_{i}}}+M C t^{\varepsilon_{i}} \sum_{j=1}^{p} \int_{0}^{1} r^{\gamma_{j}\left(\varepsilon_{j}\right)-\left(1+\varepsilon_{i}\right)} t^{\gamma_{j}\left(\varepsilon_{j}\right)-\varepsilon_{i}} d r \\
& +M C t^{\varepsilon_{i}} \sum_{j=1}^{p} \int_{0}^{t}(t-s)^{\gamma_{j}\left(\varepsilon_{j}\right)-\left(1+\varepsilon_{i}\right)} s^{-\rho_{j} \varepsilon_{j}}\left\|s^{\varepsilon_{j}} x(s)\right\|_{X^{1+\varepsilon_{j}}}^{\rho_{j}} d s \\
& \leq\left\|t^{\varepsilon_{i}} e^{-A t} x_{0}\right\|_{X^{1+\varepsilon_{i}}}+C M \sum_{j=1}^{p} B\left(1-\gamma_{j}\left(\varepsilon_{j}\right), \gamma_{j}\left(\varepsilon_{j}\right)-\varepsilon_{i}\right) \delta \\
& +t^{\varepsilon_{i}} C M \sum_{j=1}^{p} \int_{0}^{1}(t-s)^{\left(\gamma_{j}\left(\varepsilon_{j}\right)-\varepsilon_{i}\right)-1} s^{-\rho_{j} \varepsilon_{j}}\left\|s^{\varepsilon_{j}} x(s)\right\|_{X^{1+c_{j}}}^{\rho_{j}} d s \\
& +t^{\varepsilon_{i}} C M \sum_{j=1}^{p} \int_{0}^{1}(t-s)^{\left(\gamma_{j}\left(\varepsilon_{j}\right)-\varepsilon_{i}\right)-1} s^{-\rho_{j} \varepsilon_{j}}\left\|s^{\varepsilon_{j}} x(s)\right\|_{X^{1+\varepsilon_{j}}}^{\rho_{j}} d s \\
& \leq \frac{3 \mu}{4}+t^{\varepsilon_{i}} C M \sum_{j=1}^{p} \int_{0}^{1}(t-s)^{\left(\gamma_{j}\left(\varepsilon_{j}\right)-\varepsilon_{i}\right)-1} s^{-\rho_{j} \varepsilon_{j}} \mu^{\rho_{j}} d s \\
& \leq \frac{3 \mu}{4}+C M \sum_{j=1}^{p} \mu^{\rho_{j}} B_{\varepsilon_{j}}^{\varepsilon_{i}}<\mu,
\end{aligned}
$$


o que demonstra o lema.

Temos também o

Lema 1.6 Se $x \in K\left(\tau_{0}\right)$, então $T x \in C\left(\left(0, \tau_{0}\right], X^{1+\theta}\right)$, para todo $\theta \in[0, \underline{\gamma})$.

Demonstração: Fixado $t_{2} \in\left(0, \tau_{0}\right]$ seja $\tau_{0} \geq t_{1}>t_{2}$ então, para $0 \leq \theta<\underline{\gamma}$ temos

$$
\begin{aligned}
& \left\|(T x)\left(t_{1}\right)-(T x)\left(t_{2}\right)\right\|_{X^{1+\theta}} \leq\left\|\left(e^{-A t_{1}}-e^{-A t_{2}}\right) x_{0}\right\|_{X^{1+\theta}} \\
& \quad+\left\|\int_{t_{2}}^{t_{1}} e^{-A\left(t_{1}-s\right)} \sum_{i=1}^{p} H_{i}(x(s)) d s\right\|_{X^{1+\theta}} \\
& \quad+\left\|\int_{0}^{t_{2}}\left(e^{-A\left(t_{1}-s\right)}-e^{-A\left(t_{2}-s\right)}\right) \sum_{i=1}^{p} H_{i}(x(s)) d s\right\|_{X^{1+\theta}} \\
& =\left\|\left(e^{-A t_{1}}-e^{-A t_{2}}\right) x_{0}\right\|_{X^{1+\theta}}+\left\|\int_{t_{2}}^{t_{1}} e^{-A\left(t_{1}-s\right)} \sum_{i=1}^{p} H_{i}(x(s)) d s\right\|_{X^{1+\theta}} \\
& \quad+\left\|\left[-I+e^{-A\left(t_{1}-t_{2}\right)}\right] \int_{0}^{t_{2}} e^{-A\left(t_{2}-s\right)} \sum_{i=1}^{p} H_{i}(x(s)) d s\right\|_{X^{1+\theta} .}
\end{aligned}
$$

Como

$$
\left\|\left(e^{-A t_{1}}-e^{-A t_{2}}\right) x_{0}\right\|_{X^{1+\theta}} \rightarrow 0
$$

e

$$
\left\|\left[-I+e^{-A\left(t_{1}-t_{2}\right)}\right] \int_{0}^{t_{2}} e^{-A\left(t_{2}-s\right)} \sum_{i=1}^{p} H_{i}(x(s)) d s\right\|_{X^{1+\theta}} \rightarrow 0,
$$

quando $t \rightarrow 0^{+}$. Resta analisar a convergência do segundo termo. Temos

$$
\begin{aligned}
& \left\|\int_{t_{2}}^{t_{1}} e^{-A\left(t_{1}-s\right)} \sum_{i=1}^{p} H_{i}(x(s)) d s\right\|_{X^{1+\theta}} \leq M \int_{t_{2}}^{t_{1}} \sum_{i=1}^{p}\left(t_{1}-s\right)^{\gamma_{i}\left(\varepsilon_{i}\right)-(1+\theta)}\left\|H_{i}(x(s))\right\|_{X^{\gamma_{i}\left(\varepsilon_{i}\right)}} d s \\
& \leq M C \int_{t_{2}}^{t_{1}} \sum_{i=1}^{p}\left(t_{1}-s\right)^{\gamma_{i}\left(\varepsilon_{i}\right)-(1+\theta)} d s \\
& \quad+M C \int_{t_{2}}^{t_{1}} \sum_{i=1}^{p}\left(t_{1}-s\right)^{\gamma_{i}\left(\varepsilon_{i}\right)-(1+\theta)}\|x(s)\|_{X^{1+\varepsilon_{i}}}^{\rho_{i}} d s \\
& \leq M C \sum_{i=1}^{p} \int_{\frac{t_{2}}{t_{1}}}^{1}(1-r)^{\gamma_{i}\left(\varepsilon_{i}\right)-(1+\theta)} t_{1}^{\gamma_{i}\left(\varepsilon_{i}\right)-\theta} d r \\
& \quad+M C \sum_{i=1}^{p} \mu^{\rho_{i}} t_{1}^{\gamma_{i}\left(\varepsilon_{i}\right)-\left(\rho_{i}\left(\varepsilon_{i}\right)+\theta\right)} \int_{\frac{t_{2}}{t_{1}}}^{1}(1-r)^{\gamma_{i}\left(\varepsilon_{i}\right)-(1+\theta)} r^{-\rho_{i} \varepsilon_{i}} d r .
\end{aligned}
$$


Lembrando que $\theta<\underline{\gamma}<\gamma_{i}\left(\varepsilon_{i}\right), i=1,2, \cdots, p$, obtemos que

$$
\left\|\int_{t_{2}}^{t_{1}} e^{-A\left(t_{1}-s\right)} \sum_{i=1}^{p} H_{i}(x(s)) d s\right\|_{X^{1+\theta} \rightarrow 0}
$$

quando $t_{1} \rightarrow t_{2}^{+}$. O caso $t_{2}<t_{1}$ segue de forma análoga.

Lema 1.7 A aplicaçäo $T: K\left(\tau_{0}\right) \rightarrow K\left(\tau_{0}\right)$ é uma contração estrita.

Demonstração: Sejam $x, y \in C\left(\left(0, \tau_{0}\right], X^{1+\bar{\varepsilon}}\right)$ tais que

$$
\sup _{1 \leq i \leq p} \sup _{t \in\left(0, \tau_{0}\right]} t^{\varepsilon_{i}}\|x(t)\|_{X^{1+e_{i}}} \leq \mu
$$

e

$$
\sup _{1 \leq i \leq p} \sup _{t \in\left(0, \tau_{0}\right]} t^{\varepsilon_{i}}\|y(t)\|_{X^{1+e_{i}}} \leq \mu .
$$

Para cada $i=1,2, \cdots, p$ temos

$$
\begin{aligned}
& t^{\varepsilon_{i}}\left\|\int_{0}^{t} e^{-A(t-s)}\left[\sum_{j=1}^{p} H_{j}(x(s))-H_{j}(y(s))\right] d s\right\|_{X^{1+\varepsilon_{i}}} \\
& \leq t^{\varepsilon_{i}} M \int_{0}^{t} C \sum_{j=1}^{p}(t-s)^{\gamma_{j}\left(\varepsilon_{j}\right)-\left(1+\varepsilon_{i}\right)}\|x(s)-y(s)\|_{X^{1+\varepsilon_{j}}} \\
& \quad\left[\|x(s)\|_{X^{1+\varepsilon_{j}}}^{\rho_{j}-1}+\|y(s)\|_{X^{\rho_{j}-1} \varepsilon_{j}}^{\rho^{1+\varepsilon_{j}}}+1\right] d s \\
& \leq \sum_{j=1}^{p} C M\left(\int_{0}^{1} r^{\gamma_{j}\left(\varepsilon_{j}\right)-\left(1+\varepsilon_{i}\right)}(1-r)^{-\varepsilon_{j}} t^{\gamma_{j}\left(\varepsilon_{j}\right)-\varepsilon_{j}} d s\right. \\
& \left.+\int_{0}^{1} r^{\gamma_{j}\left(\varepsilon_{j}\right)-\left(1+\varepsilon_{i}\right)}(1-r)^{-\rho_{j} \varepsilon_{j}} t^{\gamma_{j}\left(\varepsilon_{j}\right)-\rho_{j} \varepsilon_{j}} 2 \mu^{\rho_{j}-1} d r\right)\|x-y\|_{K\left(\tau_{0}\right)} \\
& \leq \sum_{j=1}^{p} C M\left(\delta B_{\varepsilon_{i}}^{\varepsilon_{j}}+B_{\varepsilon ;}^{\varepsilon_{j}} 2 \mu^{\rho_{j}-1}\right)\|x-y\|_{K\left(\tau_{0}\right)} \\
& \leq \frac{3}{4}\|x-y\|_{K\left(\tau_{0}\right)},
\end{aligned}
$$

mostrando que $T$ é uma contração estrita em $K\left(\tau_{0}\right)$.

Portanto, os Lemas 1.5 e 1.7 implicam, pelo Princípio da Contração de Banach, que $T$ tem um único ponto fixo em $K\left(\tau_{0}\right)$, o qual denotaremos por $x\left(\cdot ; x_{0}\right)$, definido para todo $x_{0} \in X^{1}$ com $\left\|x_{0}-y_{0}\right\|_{X^{1}}<r$ e para todo $0 \leq t \leq \tau_{0}$. 
Observemos que o Lema 1.6 implica que

$$
x\left(\cdot ; x_{0}\right) \in C\left(\left(0, \tau_{0}\right], X^{1+\theta}\right), \quad \text { para todo } 0 \leq \theta<\underline{\gamma} .
$$

Finalmente temos o

Lema 1.8 Se $x\left(t ; x_{0}\right)$ é a solução em $K\left(\tau_{0}\right)$, então

$$
t^{\theta}\left\|x\left(t ; x_{0}\right)\right\|_{X^{1+\theta} \rightarrow 0,} \text { quando } t \rightarrow 0^{+},
$$

para todo $0 \leq \theta<\underline{\gamma}$.

Demonstração: Notemos primeiro, que com os mesmos argumentos utilizados acima, temos para todo $0<t<\tau_{0}$ e todo $0 \leq \theta<\underline{\gamma}$ que

$$
\begin{array}{r}
t^{\theta}\left\|x\left(t ; x_{0}\right)\right\|_{X^{1+\theta}} \leq t^{\theta}\left\|e^{-A t}\right\|_{X^{1+\theta}}+M C \sum_{i=1}^{p} B_{\varepsilon_{i}}^{\theta}\left(t^{\gamma_{i}\left(\varepsilon_{i}\right)}+t^{\gamma_{i}\left(\varepsilon_{i}\right)-\rho_{i} \varepsilon_{i}}\right. \\
\left.\mu^{\rho_{i}-1} \sup _{0 \leq s \leq t}\left\{s^{\varepsilon_{j}}\left\|x\left(s ; x_{0}\right)\right\|_{X^{1+\varepsilon_{j}}}\right\}\right)
\end{array}
$$

Tomando $\theta=\varepsilon_{i}$, obtemos que

$$
\begin{aligned}
t^{\varepsilon_{i}}\left\|x\left(t ; x_{0}\right)\right\|_{X^{1+\varepsilon_{i}}} \leq & t^{\varepsilon_{i}}\left\|e^{-A t} x_{0}\right\|_{X^{1+\varepsilon_{i}}}+M C \sum_{j=1}^{p} B_{\varepsilon_{j}}^{\varepsilon_{i}} \gamma^{\gamma_{j}\left(\varepsilon_{j}\right)} \\
& +M C \sum_{j=1}^{p} B_{\xi_{j}}^{\varepsilon_{i}} \mu^{\rho_{j}-1} \sup _{0 \leq s \leq t}\left\{s^{\varepsilon_{j}}\left\|x\left(s ; x_{0}\right)\right\|_{X^{1+\varepsilon_{j}}}\right\} \\
\leq & t^{\varepsilon_{i}}\left\|e^{-A t} x_{0}\right\|_{X^{1+\varepsilon_{i}}}+\frac{\mu}{4}+\frac{1}{4} \sup _{1 \leq i \leq p} \sup _{0 \leq s \leq t}\left\{s^{\varepsilon_{i}}\left\|x\left(s ; x_{0}\right)\right\|_{X^{1+\varepsilon_{i}}}\right\} .
\end{aligned}
$$

Mas, o Lema 1.4 implica que

$$
\begin{aligned}
\sup _{1 \leq i \leq p}\left\{t^{\varepsilon_{i}}\left\|x\left(t ; x_{0}\right)\right\|_{X^{1+\varepsilon_{i}}}\right\} & \leq \frac{4}{3} \sup _{1 \leq i \leq p}\left\{t^{\varepsilon_{i}}\left\|e^{-A t} x_{0}\right\|_{X^{1+\varepsilon_{i}}}\right\}+\frac{\mu}{3} \sup _{1 \leq i \leq p}\left\{t^{\varepsilon_{i}}\right\} \\
& \rightarrow 0, \text { quando } t \rightarrow 0^{+} .
\end{aligned}
$$

Portanto,

$$
\begin{aligned}
t^{\theta}\left\|x\left(t ; x_{0}\right)\right\|_{X^{1+\theta}} \leq & t^{\theta}\left\|e^{-A t} x_{0}\right\|_{X^{1+\theta}}+M C \sum_{i=1}^{p} B_{\varepsilon_{i}}^{\theta} t^{\gamma_{i}\left(\varepsilon_{i}\right)} \\
& +\left(M C \sum_{i=1}^{p} B_{\varepsilon_{i}}^{\theta} \mu^{\rho_{i}}\right) \sup _{1 \leq i \leq p 0 \leq s \leq t} \sup _{1=0}\left\{s^{\varepsilon_{j}}\left\|x\left(s ; x_{0}\right)\right\|_{X^{1+\varepsilon_{j}}}\right\} \\
\rightarrow & 0
\end{aligned}
$$


quando $t \rightarrow 0^{+}$.

Uma consequência imediata da prova do Lema 1.8 é:

$$
\begin{aligned}
\left\|x\left(t ; x_{0}\right)-x_{0}\right\|_{X^{1}} \leq & \left\|e^{-A t} x_{0}-x_{0}\right\|_{X^{1}}+M C \sum_{i=1}^{p} B_{\varepsilon_{i}}^{0} t^{\gamma_{i}\left(\varepsilon_{i}\right)} \\
& +\left(M C \sum_{i=1}^{p} B_{\varepsilon_{i}}^{0} \mu^{\rho_{i}}\right) \sup _{1 \leq i \leq p} \sup _{0 \leq s \leq t}\left\{s^{\varepsilon_{j}}\left\|x\left(s ; x_{0}\right)\right\|_{X^{1+\varepsilon_{j}}}\right\} \\
\rightarrow & 0, \text { quando } t \rightarrow 0^{+} .
\end{aligned}
$$

Com isto, temos que $x\left(t ; x_{0}\right)$ é uma solução $\bar{\varepsilon}$-regular começando em $x_{0}$, e é única no conjunto $K\left(\tau_{0}\right)$. O próximo lema será útil na prova da continuidade de soluções com relação aos dados iniciais.

Lema 1.9 Sejam $x_{0}, z_{0} \in B_{X^{1}}\left(y_{0}, r\right)$ e para $0 \leq \theta \leq \theta_{0}<\underline{\gamma}$ então,

$$
t^{\theta}\left\|x\left(t ; x_{0}\right)-x\left(t ; z_{0}\right)\right\|_{X^{1+\theta}} \leq C\left(\theta_{0}\right)\left\|x_{0}-z_{0}\right\|_{X^{1}}
$$

para alguma constante $C\left(\theta_{0}\right)>0$.

Demonstração: A prova deste lema segue de maneira análoga à prova do Lema 1.8, para uma prova detalhada veja [5] e [4].

\subsubsection{Unicidade de Soluções}

Para destacarmos a dependência das não linearidades, denotaremos uma solução do problema (1.37) por $x_{H}\left(t ; x_{0}\right)$, onde $H=\sum_{i=1}^{p} H_{i}$. Temos o

Lema 1.10 Seja $\phi(t)$ uma solução $\vec{\varepsilon}$-regular do problema (1.37) no intervalo [0, $\left.t_{0}\right]$, com dado inicial $x_{0} \in B_{X^{1}}\left(y_{0}, r\right)$ tal que para $i=1,2, \cdots, p$,

$$
t^{\varepsilon_{i}}\|\phi(t)\|_{X^{1+\epsilon_{i}} \rightarrow 0,} \text { quando } t \rightarrow 0^{+} .
$$

Então, $\phi(t)=x_{H}\left(t ; x_{0}\right)$ para $0 \leq t \leq \min \left\{\tau_{0}, t_{0}\right\}$. Ainda mais, existe $a \in\left(0, t_{0}\right]$ tal que

$$
\phi(a) \in B_{X^{1}}\left(y_{0}, r\right)
$$


e se definirmos $\phi_{a}(t):=\phi(a+t)$, para todo $t \in\left[0, t_{0}-a\right]$, então $\phi_{a} \in C\left(\left[0, t_{0}-a\right), X^{1+\bar{\varepsilon}}\right)$, $e \phi_{a}$ é uma solução $\bar{\varepsilon}$-regular do problema

$$
\begin{aligned}
& \dot{x}(t)=-A x(t)+\sum_{i=1}^{p} H_{i}(x(t)), t>a \\
& x(0)=\phi(a) .
\end{aligned}
$$

Demonstração: Como $\phi(t)$ é uma solução $\bar{\varepsilon}$-regular então,

$$
\phi \in C\left(\left[0, t_{0}\right], X^{1}\right) \cap C\left(\left(0, t_{0}\right], X^{1+\bar{\varepsilon}}\right) .
$$

Ainda, tomando $\bar{\tau} \leq \tau_{0}$ e $\bar{\tau} \leq t_{0}$ suficientemente pequeno tal

$$
t^{\varepsilon_{i}}\|\phi(t)\|_{X^{1+\varepsilon_{i}}} \leq \mu,
$$

para $0<t \leq \bar{\tau}$ e para $i=1,2, \cdots, p$, obtemos que $\phi \in K(\bar{\tau})$. Mas $x_{H}\left(\cdot ; x_{0}\right) \in K(\bar{\tau}) \mathrm{e}$ ambas satisfazem a equação integral (1.40), logo

$$
x_{H}\left(t ; x_{0}\right)=\phi(t) \text { para } t \in[0, \bar{\tau}] .
$$

Um argumento padrão de continuação implica que

$$
x_{H}\left(t ; x_{0}\right)=\phi(t) \text { para } t \in\left[0, \min \left\{\tau_{0}, t_{0}\right\}\right] .
$$

A continuidade de $\phi$ em $X^{1}$ implica que existe $a \in\left(0, t_{0}\right]$ tal que $\phi(a) \in B_{X^{1}}\left(y_{0}, r\right)$.

Para mostrarmos a outra parte, notemos primeiro que

$$
\phi_{a} \in C\left(\left[0, t_{0}-a\right], X^{1+\bar{\varepsilon}}\right) .
$$

Logo, $\phi_{a}(0)=\phi(a) \in B_{X^{1}}\left(y_{0}, r\right)$, e existem constantes positivas $\bar{\delta}$ e $K$ tais que

$$
\left\|\phi_{a}(t)\right\|_{X^{1+\varepsilon_{i}}} \leq K
$$

para todo $t \in[0, \bar{\delta}]$, e $i=1,2, \cdots, p$. Portanto

$$
t^{\varepsilon_{i}}\left\|\phi_{a}(t)\right\|_{X^{1+\epsilon_{i}}} \rightarrow 0, \text { quando } t \rightarrow 0^{+} .
$$

Com um raciocínio simples mostramos que

$$
\phi(t+a)=e^{-A t} \phi(a)+\int_{0}^{t} e^{-A(t-s)} \sum_{i=1}^{p} H_{i}\left(\phi_{a}(s)\right) d s .
$$


Assim, $\phi_{a}$ é uma solução $\bar{\varepsilon}$-regular do problema (1.46).

Para mostrarmos a unicidade de soluções, seja $\phi(t)$ uma solução $\bar{\varepsilon}$-regular do problema (1.37) no intervalo $\left[0, t_{0}\right]$, começando em $x_{0} \in B_{X^{1}}\left(y_{0}, r\right)$, a prova do Lema 1.10 e a parte de existência de soluções implicam que existe uma única solução do problema (1.46) no conjunto $K\left(\tau_{0}\right)$, a qual denotaremos por $x(t ; \phi(a))$, definida em $[0, \tau]$. O Lema 1.10 implica que

$$
x(t ; \phi(a))=\phi_{a}(t), 0 \leq t \leq \min \left\{\tau_{0}, t_{0}-a\right\}
$$

para todo $a \in\left(0, a_{0}\right]$.

Em particular, podemos assumir, sem perda da generalidade, que $t_{0} \geq \tau_{0}$, pois caso contrário, definindo

$$
\begin{array}{ll}
\tilde{\phi}(t)=\phi(t), & 0 \leq t \leq t_{0} \\
\tilde{\phi}(t)=x\left(t-a_{0}, \phi\left(a_{0}\right)\right), & t_{0} \leq t \leq \tau_{0}
\end{array}
$$

os resultados acima mostram que $\tilde{\phi}$ é também uma solução $\bar{\varepsilon}$-regular de (1.37), começando em $x_{0}$.

Mas, para todo $0<a<a_{0}$ e para $0<t<t_{0}$ fixado, temos

$$
\begin{aligned}
t^{\varepsilon_{i}}\|\phi(t)\|_{X^{1+\varepsilon_{i}}} & \leq t^{\varepsilon_{i}}\|\phi(t)-\phi(t+a)\|_{X^{1+\varepsilon_{i}}}+t^{\varepsilon_{i}}\left\|\phi_{a}(t)\right\|_{X^{1+\varepsilon_{i}}} \\
& \leq t^{\varepsilon_{i}}\|\phi(t)-\phi(t+a)\|_{X^{1+\varepsilon_{i}}}+t^{\varepsilon_{i}}\left\|x\left(t ; \phi_{a}\right)\right\|_{X^{1+\varepsilon_{i}}} \\
& \leq t^{\varepsilon_{i}}\|\phi(t)-\phi(t+a)\|_{X^{1+\varepsilon_{i}}}+\mu .
\end{aligned}
$$

Fazendo $a \rightarrow 0^{+}$, obtemos que

$$
t^{\epsilon_{i}}\|\phi(t)\|_{X^{1+\varepsilon_{i}}} \leq \mu
$$

para $i=1,2, \cdots, p$ e para todo $t \in\left[0, \tau_{0}\right]$, provando a unicidade de soluções .

\subsubsection{Prova do Teorema $\mathbf{1 . 9}$}

As duas Subseções anteriores, mostram a existência e unicidade de soluções, bem como (1.42). 
Para mostrarmos a regularidade extra afirmada no teorema, observemos primeiro que,

$$
H:=\sum_{i=1}^{p} H_{i}: X^{1+\vec{\varepsilon}} \rightarrow X^{\underline{\gamma}},
$$

é localmente Lipschitziana em subconjuntos limitados. Logo o Lema 3.3.2 de [17] implica que

$$
x \in C\left(\left(0, \tau_{0}\right], X^{1+\underline{\gamma}}\right) .
$$

Também, o Teorema 3.5.2 de [17] implica que

$$
x_{t} \in C\left(\left(0, \tau_{0}\right], X^{1+\theta}\right),
$$

para todo $\theta \in[0, \underline{\gamma})$.

Concluímos assim a prova do Teorema 1.9.

O próximo resultado será útil para mostramos a existência global de soluções do problema (1.1), e sua prova pode ser encontrada com detalhes em [5].

Proposição 1.4 Sejam $F_{i}, i=1,2, \cdots, p$ como no Teorema 1.9 ex $x\left(t ; x_{0}\right)$ uma solução $\vec{\varepsilon}$-regular começando em $x_{0}$, com tempo maximal de existência $\tau_{m}<\infty$, então

$$
\lim _{t \rightarrow \tau_{m}^{-}}\left\|x\left(t ; x_{0}\right)\right\|_{X^{1+\delta}}=\infty, 0<\delta<\bar{\varepsilon},
$$

onde $\bar{\varepsilon}:=\min \left\{\varepsilon_{i}, i=1,2, \cdots, p\right\}$. Se além disso, $\gamma_{i}\left(\varepsilon_{i}\right)>\rho_{i} \varepsilon_{i}$, para todo $i=1,2, \cdots, p$, então

$$
\lim _{t \rightarrow \tau_{m}^{-}}\left\|x\left(t ; x_{0}\right)\right\|_{X^{1}}=\infty
$$

Como em [5], classificamos uma função $\varepsilon$-regular relativamente ao par $\left(X^{1}, X^{0}\right)$, para $\varepsilon$ em um intervalo $I$, da seguinte maneira:

- Se $I=\left[0, \varepsilon_{1}\right]$ para $\varepsilon_{1}>0$ e $\gamma(0)>0$. Diremos que $f$ é uma aplicação subcrítica em relaçāo ao par $\left(X^{1}, X^{0}\right)$.

- Se $I=\left[0, \varepsilon_{1}\right]$ para $\varepsilon_{1}>0 \operatorname{com} \gamma(\varepsilon)=\rho \varepsilon, \varepsilon \in I$ e se $f$ não é subcrítica, então diremos que $f$ é uma aplicação crítica em relação ao par $\left(X^{1}, X^{0}\right)$. 
- Se $I=\left(0, \varepsilon_{1}\right]$ para $\varepsilon_{1}>0$ com $\gamma(\varepsilon)=\rho \varepsilon, \varepsilon \in I$ e se $f$ não é subcrítica e nem crítica, então diremos que $f$ é uma aplicação duplamente-crítica em relação ao $\operatorname{par}\left(X^{1}, X^{0}\right)$.

- Se $I=\left[\varepsilon_{0}, \varepsilon_{1}\right]$ para $\varepsilon_{1}>\varepsilon_{0}>0$ com $\gamma\left(\varepsilon_{0}\right)>\rho \varepsilon_{0}$ e se $f$ não é subcrítica, nem crítica e nem duplamente-crítica, então diremos que $f$ é uma aplicação ultrasubcrítica em relação ao par $\left(X^{1}, X^{0}\right)$.

- Se $I=\left[\varepsilon_{0}, \varepsilon_{1}\right]$ para $\varepsilon_{1}>\varepsilon_{0}>0$ com $\gamma(\varepsilon)=\rho \varepsilon$ e se $f$ não é subcrítica, nem crítica, nem duplamente-crítica e nem ultra-crítica, então diremos que $f$ é uma aplicação ultra-crítica em relação ao par $\left(X^{1}, X^{0}\right)$.

\subsection{Existência e Unicidade de Soluções para o Pro- blema (1.1)}

Nesta seção usaremos os resultados das seções anteriores para mostrarmos que o problema (1.1) possui uma única solução com dado inicial em $L^{q}\left(\Omega, \mathbb{R}^{n}\right)$, ou em $W^{1, q}\left(\Omega, \mathbb{R}^{n}\right)$. Seguiremos nesta seção as idéias contidas em [5].

A principal razão para trabalharmos com a escala de espaços de Banach contruída na Seção 1.1, é que podemos resolver o problema (1.1) nos dois sentidos abaixo:

Definição 1.11 Para $u_{0} \in W^{1, q}\left(\Omega, \mathbb{R}^{n}\right)$, uma solução variacional fraca do problema (1.1), é uma função $u \in C\left([0, \tau), W^{1, q}\left(\Omega, \mathbb{R}^{n}\right)\right)$ tal que

$$
\frac{d}{d t} \int_{\Omega} u \phi+\int_{\Omega} \nabla u \nabla \phi=\int_{\Omega} F(u(x)) \phi+\int_{\Gamma} G(u(x)) \phi,
$$

para toda $\phi \in W^{1, q^{\prime}}\left(\Omega, \mathbb{R}^{n}\right)$, onde $F=\left(F_{1}, \cdots, F_{n}\right)$ e $G=\left(G_{1}, \cdots, G_{n}\right)$ são definidas, para $v=\left(v_{1}, v_{2}, \cdots, v_{n}\right) \in \mathbb{R}^{n}$, por

$$
\begin{aligned}
& F_{i}(v)=f_{i}(v), \quad i=1,2, \cdots, n, \\
& G_{1}(v)=g_{1}(v)-k\left(v_{1}-v_{2}\right), \\
& G_{2}(v)=g_{2}(v)+k\left(v_{1}-v_{2}\right), \\
& G_{i}(v)=g_{i}(v), \quad i=3,4, \cdots, n, \text { se } n>2,
\end{aligned}
$$

onde $f_{i}, g_{i}: \mathbb{R}^{n} \rightarrow \mathbb{R}$ são funções localmente lipschitzianas. 
Definição 1.12 Para $u_{0} \in L^{q}\left(\Omega, \mathbb{R}^{n}\right)$, uma solução variacional muito fraca do problema (1.1), é uma função $u \in C\left([0, \tau), L^{q}\left(\Omega, \mathbb{R}^{n}\right)\right)$ tal que

$$
\frac{d}{d t} \int_{\Omega} u \phi-\int_{\Omega} u \triangle \phi=\int_{\Omega} F(u(x)) \phi+\int_{\Gamma} G(u(x)) \phi,
$$

para $\phi \in W_{\mathcal{N}}^{2, q^{\prime}}\left(\Omega, \mathbb{R}^{n}\right)$, onde $W_{\mathcal{N}}^{2, q^{\prime}}\left(\Omega, \mathbb{R}^{n}\right)$ é o conjunto de funções em $W^{2, q^{\prime}}\left(\Omega, \mathbb{R}^{n}\right)$, com derivada normal igual a zero em $\Gamma, F$ e $G$ são como definidas em (1.48).

Para aplicarmos os resultados da Seção 1.2, precisamos escrever o problema (1.1) em uma formulação abstrata, como em (1.37). Consideremos o operador $A$ como definido na Subseção 1.1.7, e sejam

$$
\left\{\left(E_{q}^{\alpha}, A_{\alpha}\right) ; \alpha \in \mathbb{R}\right\},
$$

para $1<q<\infty$, a escala de espaços de Banach, como contruída na Subseção 1.1.7. Temos, pelo Teorema 1.6, que

$$
E_{q}^{-\alpha}=\left(E_{q^{\prime}}^{\alpha}\right)^{\prime}
$$

para todo $\alpha>0$. Ainda,

$$
E_{q}^{-\frac{1}{2}}=\left(W^{1, q^{\prime}}\left(\Omega, \mathbb{R}^{n}\right)\right)^{\prime}=: W^{-1, q}\left(\Omega, \mathbb{R}^{n}\right) .
$$

Se considerarmos as realizações de $A$ sobre essa escala, temos que

$$
A_{-\frac{1}{2}} \in \mathcal{L}\left(E_{q}^{\frac{1}{2}}, E_{q}^{-\frac{1}{2}}\right)
$$

e é dado, para todo $u \in E_{q}^{1 / 2}$, por

$$
\left\langle A_{-\frac{1}{2}} u, \phi\right\rangle=-\int_{\Omega} D \nabla u . \nabla \phi,
$$

para toda $\phi \in W^{1, q^{\prime}}\left(\Omega, \mathbb{R}^{n}\right)$, onde $D$ é a matriz dada em (1.2).

Por outro lado, o operador

$$
A_{-1} \in \mathcal{L}\left(E_{q}^{0}, E_{q}^{-1}\right)
$$

e é dado, para todo $u \in E_{q}^{0}$, por

$$
\left\langle A_{-1} u, \phi\right\rangle=-\int_{\Omega} D u . \nabla \phi,
$$


para toda $\phi \in W_{\mathcal{N}}^{2, q^{\prime}}\left(\Omega, \mathbb{R}^{n}\right)$, onde $W_{\mathcal{N}}^{2, q^{\prime}}\left(\Omega, \mathbb{R}^{n}\right)$ é definido como em (1.34) e $D$ é a matriz definida em (1.2).

Com isso em mente, podemos reescrever o problema (1.1) da seguinte forma:

$$
\begin{aligned}
& \dot{u}(t)=-A_{-1 / 2} u(t)+H u(t), t>0 \\
& u(0)=u_{0} \in W^{1, q}\left(\Omega, \mathbb{R}^{n}\right),
\end{aligned}
$$

o qual corresponde à formulação fraca (1.47). Ou, podemos reescrever o problema (1.1) como

$$
\begin{aligned}
& \dot{u}(t)=-A_{-1} u(t)+H u(t), \quad t>0 \\
& u(0)=u_{0} \in L^{q}\left(\Omega, \mathbb{R}^{n}\right),
\end{aligned}
$$

que corresponde à formulação muito fraca (1.49).

Em ambos os casos, $H$ é definida por $H(u):=F_{\Omega}(u)+G_{\Gamma}(u)$, atuando em adequadas funções testes $\phi$ da seguinte maneira:

$$
\left\langle F_{\Omega}(u)+G_{\Gamma}(u), \phi\right\rangle:=\int_{\Omega} F(u(x)) \cdot \phi(x)+\int_{\Gamma} G(u(x)) \cdot \phi(x),
$$

onde $F, G$ são como definidas em (1.48).

Para aplicarmos os resultados da Seção 1.2, precisamos usar algumas imersões já conhecidas. Sejam $\Omega \subset \mathbb{R}^{N}$ um domínio com fronteira suave, $1<q<\infty$ e $l \geq 0$. Consideremos $H_{q}^{l}\left(\Omega, \mathbb{R}^{n}\right)$ os espaços de potências de Bessel, para maiores detalhes, veja [7]. Ainda,

$$
H_{q}^{-l}\left(\Omega, \mathbb{R}^{n}\right):=\left(H_{q^{\prime}}^{l}\left(\Omega, \mathbb{R}^{n}\right)\right)^{\prime}, 1<q<\infty \quad l \geq 0 .
$$

Para $1<q<\infty$, temos as seguintes imersōes:

$$
\begin{array}{ll}
H_{q}^{l}\left(\Omega, \mathbb{R}^{n}\right) \subset L^{r}\left(\Omega, \mathbb{R}^{n}\right) & \text { se } \frac{l}{N}-\frac{1}{q} \geq-\frac{1}{r} \\
H_{q}^{l}\left(\Omega, \mathbb{R}^{n}\right) \subset \mathcal{C}^{\eta}\left(\bar{\Omega}, \mathbb{R}^{n}\right) & \text { se } l-\frac{N}{q}>\eta>0,
\end{array}
$$

com imersões contínuas. A prova dessas imersões podem ser encontradas em $[1,7]$.

Ainda mais, se $T$ denota o operador traço, então para $l>\frac{1}{q}, 1<q<\infty, T$ é bem definido em $H_{q}^{l}\left(\Omega, \mathbb{R}^{n}\right)$ e

$$
H_{q}^{l}\left(\Omega, \mathbb{R}^{n}\right) \stackrel{T}{\longrightarrow} L^{r}\left(\Gamma, \mathbb{R}^{n}\right) \quad \text { se } q\left\{\begin{array}{lll}
\leq \infty & \text { se } & l q>N \\
<\infty & \text { se } & l q=N \\
\leq \frac{(N-1) q}{N l-q} & \text { se } & l q<N
\end{array}\right.
$$


Portanto, usando (1.35) e (1.54), concluímos que

$$
\begin{array}{ll}
E_{q}^{\alpha} \hookrightarrow L^{r}\left(\Omega, \mathbb{R}^{n}\right), \quad r \leq \frac{N q}{N-2 \alpha q}, & 0 \leq \alpha<\frac{N}{2 q} \\
E_{q}^{0}=L^{q}\left(\Omega, \mathbb{R}^{n}\right) & \\
E_{q}^{\alpha} \hookleftarrow L^{s}\left(\Omega, \mathbb{R}^{n}\right), \quad s \geq \frac{N q}{N-2 \alpha q}, & -\frac{N}{2 q^{\prime}}<\alpha \leq 0,
\end{array}
$$

para $1<q<\infty$, com imersões contínuas.

\subsubsection{Existência e Unicidade de Soluções em $L^{q}\left(\Omega, \mathrm{IR}^{n}\right)$}

Nesta subseção mostraremos que o problema (1.1) possui uma única solução em $L^{q}\left(\Omega, \mathbb{R}^{n}\right)$. Para isso, precisamos assumir que as não linearidades em (1.1) satisfaçam algumas condiçoes de crescimentos, as quais passamos a enunciar agora. Para todo $i=1,2, \cdots, n$ suponhamos que $f_{i}$ satisfaça

$$
\left|f_{i}(u)-f_{i}(v)\right| \leq c|u-v|\left(|u|^{\rho_{i}-1}+|v|^{\rho_{i}-1}+1\right), \quad u, v \in \mathbb{R}^{n} .
$$

$\mathrm{E} g_{i}, i=1,2, \cdots, n$ satisfaça

$$
\left|g_{i}(u)-g_{i}(v)\right| \leq c|u-v|\left(|u|^{\bar{\rho}_{i}-1}+|v|^{\bar{\rho}_{i}-1}+1\right), \quad u, v \in \mathbb{R}^{n} .
$$

Como as notaçoes do começo desta seção, definamos para $1<q<\infty$,

$$
X_{q}^{\alpha}:=E_{q}^{\alpha-1}, \quad \alpha \in \mathbb{R}
$$

e

$$
A_{q}: X_{q}^{1} \subset X_{q}^{0} \rightarrow X_{q}^{0}
$$

a $E_{q}^{-1}$-realização de $A$ definida em (1.52). Com isso, (1.56) implica que

$$
\begin{aligned}
& X_{q}^{\alpha} \hookrightarrow L^{r}\left(\Omega, \mathbb{R}^{n}\right), \quad r \leq \frac{N q}{N+2 q-2 \alpha q}, \quad 1 \leq \alpha<\frac{N}{2 q}+1 \\
& X_{q}^{1}=L^{q}\left(\Omega, \mathbb{R}^{n}\right) \\
& X_{q}^{\alpha} \hookleftarrow L^{s}\left(\Omega, \mathbb{R}^{n}\right), \quad s \geq \frac{N q}{N+2 q-2 \alpha q}, \quad 1-\frac{N}{2 q^{\prime}}<\alpha \leq 1,
\end{aligned}
$$

onde $1<q<\infty$, com imersões contínuas. Neste contexto, obteremos uma solução do problema (1.1) no sentido de (1.49). Na realidade, essa solução é clássica.

Lema 1.11 Suponhamos que $f_{i}, i=1,2, \cdots, n$ satisfaçam as condições de crescimento afirmadas acima. Então para $\varepsilon>0$ e $1<\rho_{i} \leq \rho_{1}, i=1,2, \cdots, n$ como acima temos

$$
\left\|F_{\Omega}(u)-F_{\Omega}(v)\right\|_{X^{\gamma(\varepsilon)}} \leq C\|u-v\|_{X^{1+\varepsilon}}\left(\|u\|_{X^{1+\varepsilon}}^{\rho_{1}-1}+\|v\|_{X^{1+\varepsilon}}^{\rho_{1}-1}+1\right),
$$

onde $\gamma(\varepsilon):=1+\frac{N}{2 q}\left(1-\rho_{1}\right)+\varepsilon \rho_{1}$, e $F_{\Omega}$ é como definida em (1.53). 
Demonstração: Seja $u \in L^{\rho_{1} r}\left(\Omega, \mathbb{R}^{n}\right)$. Para $i=1,2, \cdots, n$, usando a a condição de crescimento afirmada acima, temos

$$
\begin{aligned}
\left(\int_{\Omega}\left|f_{i}(u(x))\right|^{r} d x\right)^{1 / r} & \leq\left(\int_{\Omega}\left(c\left(|u(x)|^{\rho_{1}}+1\right)\right)^{r} d x\right)^{1 / r} \\
& \leq c\left(\left(\int_{\Omega}|u(x)|^{\rho_{1} r}\right)^{1 / r}+|\Omega|^{1 / r}\right) \\
& =c\left(\|u\|_{L^{\rho_{1} r}(\Omega)}+1\right),
\end{aligned}
$$

lembrando que $F(v)=\left(f_{1}(v), f_{2}(v), \cdots, f_{n}(v)\right)$, para todo $v \in \mathbb{R}^{n}$, obtemos que $F_{\Omega}$ : $L^{\rho_{1} r}\left(\Omega, \mathbb{R}^{n}\right) \rightarrow L^{r}\left(\Omega, \mathbb{R}^{n}\right)$ é bem definida para todo $r \geq 1$.

Da mesma forma, mostramos que para todos $u, v \in L^{\rho_{1 r}}\left(\Omega, \mathbb{R}^{n}\right)$ satisfaz

$$
\left\|F_{\Omega}(u)-F_{\Omega}(v)\right\|_{L^{r}\left(\Omega, \mathbb{R}^{n}\right)} \leq c\|u-v\|_{L^{\rho_{1} r}\left(\Omega, \mathbb{R}^{n}\right)}\left(\|u\|_{L^{\rho_{1} r}\left(\Omega, \mathbb{R}^{n}\right)}^{\rho_{1}-1}+\|v\|_{L^{\rho_{1} r}\left(\Omega, \mathbb{R}^{n}\right)}^{\rho_{1}-1}+1\right),
$$

de onde obtemos, em particular, que $F: L^{\rho_{1} r}\left(\Omega, \mathbb{R}^{n}\right) \rightarrow L^{r}\left(\Omega, \mathbb{R}^{n}\right)$ é uma aplicação lipschitziana em subconjuntos limitados de $L^{\rho_{1} r}\left(\Omega, \mathbb{R}^{n}\right)$, para todo $r \geq 1$.

Agora, (1.57) implica que

$$
X_{q}^{1+\varepsilon} \hookrightarrow L^{\rho_{1} r(\varepsilon)}\left(\Omega, \mathbb{R}^{n}\right)
$$

se

$$
r(\varepsilon)=\frac{N q}{(N-2 q \varepsilon) \rho_{1}} \quad \text { e } \quad 0 \leq \varepsilon<\frac{N}{2 q} .
$$

Novamente, (1.57) implica que

$$
X_{q}^{\gamma} \hookleftarrow L^{r(\varepsilon)}\left(\Omega, \mathbb{R}^{n}\right),
$$

se

$$
r(\varepsilon)=\frac{N q}{N+2 q-2 \gamma q} \quad \text { e } \quad 1-\frac{N}{2 q^{\prime}}<\gamma \leq 1 .
$$

Substituindo o valor de $r(\varepsilon)$ encontrado acima, obtemos que

$$
X_{q}^{\gamma(\varepsilon)} \hookleftarrow L^{r(\varepsilon)}\left(\Omega, \mathbb{R}^{n}\right),
$$

para $\gamma(\varepsilon)=1+\frac{\gamma}{2 q}\left(1-\rho_{1}\right)+\rho_{1} \varepsilon$ e $\varepsilon \in\left(0, \frac{N}{2 q}\right)$. 
Temos a seguinte cadeia

$$
X_{q}^{1+\varepsilon} \hookrightarrow\left(\Omega, \mathbb{R}^{n}\right) \stackrel{F_{n}}{\longrightarrow} L^{r(\varepsilon)}\left(\Omega, \mathbb{R}^{n}\right) \hookrightarrow X^{\gamma(\varepsilon)},
$$

para $\varepsilon, r(\varepsilon)$ e $\gamma(\varepsilon)$ satisfazendo as condições acima, o que prova o lema.

Observemos que da prova do Lema 1.11, se $q>\rho_{1}$, então $\varepsilon$ pode ser tomando igual a zero, e se $q=\rho_{1}$, então $\varepsilon$ deve ser tomado estritamente positivo. Também, se $N=1,2$ ou $N \geq 3$ e $1<q<\rho_{1}$, devemos tomar $\varepsilon>\varepsilon_{0}:=\frac{1}{\rho_{1}}\left(1-\frac{N}{2 q^{\prime}}\right)$. Ainda, como pela Definição 1.2 devemos ter $\rho_{1} \varepsilon \leq \gamma(\varepsilon)<1$, encontramos as seguintes estimativas para $\varepsilon$ e $\rho_{1}$ :

$$
\varepsilon \in\left(0, \frac{\rho_{1}-1}{\rho_{1}} \frac{N}{2 q}\right) \quad \text { e } \quad \rho_{1} \leq \frac{N+2 q}{N} .
$$

Com essas observações e o Lema 1.11, podemos provar as duas próximas proposições.

Proposição 1.5 (Caso Crítico) Sejam $1<q<\infty e \rho_{1}=\rho_{f}=\frac{N+2 q}{N}$. Então

- Se $N \geq 3, q>\frac{N}{N-2}$, então $F_{\Omega}$ é uma aplicação $\varepsilon$-regular relativamente ao par $\left(X_{q}^{1}, X_{q}^{0}\right)$ para $0 \leq \varepsilon<\frac{1}{\rho_{1}}$ e $\gamma(\varepsilon)=\rho_{1} \varepsilon$.

- Se $N \geq 3, q=\frac{N}{N-2}$, então $F_{\Omega}$ é uma aplicação $\varepsilon$-regular relativamente ao par $\left(X_{q}^{1}, X_{q}^{0}\right)$ para $0<\varepsilon<\frac{1}{\rho_{1}}$ e $\gamma(\varepsilon)=\rho_{1} \varepsilon$.

- Se $N=1,2$ e $1<q<\infty$ ou $N \geq 3$, e $1<q<\frac{N}{N-2}$, então $F_{\Omega}$ é uma aplicação $\varepsilon-$ regular relativamente ao par $\left(X_{q}^{1}, X_{q}^{0}\right)$ para $0<\frac{1}{\rho_{1}}\left(1-\frac{N}{2 q^{\prime}}\right)<\varepsilon<\frac{1}{\rho_{1}}$ e $\gamma(\varepsilon)=\rho_{1} \varepsilon$.

Proposição 1.6 (Caso Subcrítico) Sejam $1<q<\infty e \rho_{1}<\rho_{f}=\frac{N+2 q}{N}$. Então

- Se $q \geq \frac{N}{N-2}$, então $F_{\Omega}$ é uma aplicação subcrítica relativamente ao par $\left(X_{q}^{1}, X_{q}^{0}\right)$.

- Se $1<q<\frac{N}{N-2}$ e $\rho_{1} \leq q$, então $F_{\Omega}$ é uma aplicação subcrítica relativamente ao $\operatorname{par}\left(X_{q}^{1}, X_{q}^{0}\right)$.

- Se $1<q<\frac{N}{N-2}$ e $N / 2\left(\rho_{1}-1\right)<q<\rho_{1}$, então $F_{\Omega}$ é uma aplicação ultra-subcrítica relativamente ao par $\left(X_{q}^{1}, X_{q}^{0}\right)$.

Com relaçāo à não linearidade $G_{\Gamma}$, temos o seguinte lema: 
Lema 1.12 Suponhamos que $g_{i}, i=1,2, \cdots, n$ satisfaçam as condições de crescimento afirmadas acima. Então para $\varepsilon>0$ e $1<\bar{\rho}_{i}<\rho_{2}, i=1,2, \cdots, n$, como acima temos

$$
\left\|G_{\Gamma}(u)-G_{\Gamma}(v)\right\|_{X^{\gamma(\varepsilon)}} \leq C\|u-v\|_{X^{1+e}}\left(\|u\|_{X^{1+\varepsilon}}^{\rho_{2}-1}+\|v\|_{X^{1+\varepsilon}}^{\rho_{2}-1}+1\right),
$$

onde $\gamma(\varepsilon):=\frac{1}{2}+\frac{N}{2 q}\left(1-\rho_{2}\right)+\varepsilon \rho_{2}$, e $G_{\Gamma}$ é como definida em (1.53).

Demonstração: Da mesma forma que na prova do Lema 1.11, podemos mostrar que

$$
G_{\Gamma}: L^{\rho_{2} r}\left(\Gamma, \mathbb{R}^{n}\right) \rightarrow L^{r}\left(\Gamma, \mathbb{R}^{n}\right),
$$

é uma aplicação bem definida tal que, para todos $u, v \in L^{\rho_{2} r}\left(\Gamma, \mathbb{R}^{n}\right)$,

$$
\left\|G_{\Gamma}(u)-G_{\Gamma}(v)\right\|_{L^{r}\left(\Gamma, \mathbb{R}^{n}\right)} \leq c\|u-v\|_{L^{\rho_{2} r}\left(\Gamma, \mathbb{R}^{n}\right)}\left(\|u\|_{L^{\rho_{2} r}\left(\Gamma, \mathbb{R}^{n}\right)}^{\rho^{\rho^{2}-1}}+\|v\|_{L^{\rho^{r}\left(\Gamma, \mathbb{R}^{n}\right)}}^{\rho_{2}-1}+1\right) .
$$

Portanto, $G$ é uma aplicação lipichitziana em subconjunto limitados de $L^{\rho_{2} r}\left(\Gamma, \mathbb{R}^{n}\right)$, para todo $r \geq 1$.

Recordemos que para todo $0 \leq \varepsilon \leq 1$, temos

$$
X_{q}^{1+\varepsilon} \hookrightarrow H_{q}^{2 \varepsilon}\left(\Omega, \mathbb{R}^{n}\right)
$$

Por outro lado, (1.55) implica que

$$
H_{q}^{2 \varepsilon}\left(\Omega, \mathbb{R}^{n}\right) \stackrel{T}{\rightarrow} L^{\rho_{2} r(\varepsilon)}\left(\Gamma, \mathbb{R}^{n}\right)
$$

se

$$
\rho_{2} r(\varepsilon) \leq \frac{(N-1) q}{N-2 q \varepsilon} \quad \text { e } \quad \varepsilon<N / 2 q .
$$

Mas uma condição para o operador traço, $T$, estar bem definida é dada por $\rho_{2} r(\varepsilon)>q$.

Lembrando sempre que devemos ter $\gamma(\varepsilon) \geq \rho_{2} \varepsilon,(1.35)$ e (1.55) implicam que

$$
\left(X_{q}^{\gamma(\varepsilon)}\right)^{\prime} \hookrightarrow H_{q^{\prime}}^{2-\rho_{2} 2 \varepsilon}\left(\Omega, \mathbb{R}^{n}\right) \stackrel{T}{\rightarrow} L^{r(\varepsilon)^{\prime}}\left(\Gamma, \mathbb{R}^{n}\right)
$$

se

$$
q>\frac{(N-1) q}{(N-2 q \varepsilon) \rho_{2}}>1
$$

onde as desigualdades acima são obtidas através das restrições para o operador traço, $T$, estar bem definido. Substituindo as restrições de $\varepsilon, r(\varepsilon)$ obtemos que

$$
\gamma(\varepsilon):=\frac{1}{2}+\frac{N}{2 q}\left(1-\rho_{2}\right)+\varepsilon \rho_{2} .
$$


Agora, para toda $\phi \in\left(X_{q}^{\gamma(\varepsilon)}\right)^{\prime}$ e todo $u, v \in X_{q}^{1+\varepsilon}$, temos

$$
\begin{aligned}
& \left|\left(G_{\Gamma}(u)-G_{\Gamma}(v), \phi\right\rangle\right| \leq\left\|G_{\Gamma}(u)-G_{\Gamma}(v)\right\|_{L^{r(\varepsilon)}\left(\Gamma, \mathbb{R}^{n}\right)}\|\phi\|_{L^{r(\varepsilon)^{\prime}}\left(\Gamma, \mathbb{R}^{n}\right)} \\
& \leq c\|u-v\|_{L^{\rho_{2} r}\left(\Gamma, \mathbb{R}^{n}\right)}\left(\|u\|_{L^{\rho_{2} r}\left(\Gamma, \mathbb{R}^{n}\right)}^{\rho_{2}-1}+\|v\|_{L^{\rho_{2} r}\left(\Gamma, \mathbb{R}^{n}\right)}^{\rho_{2}-1}+1\right)\|\phi\|_{L^{r(\varepsilon)^{\prime}}\left(\Gamma, \mathbb{R}^{n}\right)} \\
& \leq c\|u-v\|_{X_{q}^{1+\varepsilon}}\left(\|u\|_{X_{q}^{1+\varepsilon}}^{\rho_{2}-1}+\|v\|_{X_{q}^{1+\varepsilon}}^{\rho_{2}-1}+1\right)\|\phi\|_{\left(X_{q}^{\gamma(\varepsilon)}\right)^{\prime}\left(\Gamma, \mathbb{R}^{n}\right)}
\end{aligned}
$$

para $\varepsilon, r(\varepsilon)$ e $\gamma(\varepsilon)$ satisfazendo as restrições acima, demonstrando assim o lema.

O Lema 1.12, juntamente com as restrições sobre $\varepsilon, \gamma(\varepsilon)$ e $r(\varepsilon)$ encontradas em sua prova mostram as duas próximas proposições.

Proposição 1.7 (Caso Crítico) Sejam $N>1,1<q<\infty e \rho_{2}=\rho_{g}=\frac{N+q}{N}$. Então

- Se $1<q<\frac{N}{N-1}$, então $G_{\Gamma}$ é uma aplicação $\varepsilon$-regular relativamente ao par $\left(X_{q}^{1}, X_{q}^{0}\right)$ para $\frac{1}{\rho_{2}}\left(1-\frac{N}{2 q^{\prime}}\right)<\varepsilon<\frac{1}{2 \rho_{2}}\left(\frac{q+1}{q}\right)$ e $\gamma(\varepsilon)=\rho_{2} \varepsilon$.

- Se $q \geq \frac{N}{N-1}$, então $G_{\Gamma}$ é uma aplicação E-regular relativamente ao par $\left(X_{q}^{1}, X_{q}^{0}\right)$ para $\frac{1}{2 q}<\varepsilon<\frac{1}{2 \rho_{2}}\left(\frac{q+1}{q}\right)$ e $\gamma(\varepsilon)=\rho_{2} \varepsilon$.

Proposição 1.8 (Caso Subcrítico) Sejam $N \geq 1,1<q<\infty e \rho_{2}<\rho_{g}=\frac{N+q}{N}$. Então $G_{\Gamma}$ é uma aplicação ultra subcrítica.

Como sempre estaremos nos referindo às condiçōes de crescimento das funçōes $f_{i}, g_{i}$, $i=1,2, \cdots, n$ resumimos os resultados das Proposições $1.5-1.8$ da seguinte maneira:

(C1) Suponhamos que $f_{i}, g_{i}, i=1,2, \cdots, n$ satisfaçam uma relação da forma

$$
|h(u)-h(v)| \leq c|u-v|\left(|u|^{\rho-1}+|v|^{\rho-1}+1\right), \quad u, v \in \mathbb{R}^{n}
$$

com expoentes $\rho_{i}$ e $\overline{\rho_{i}}, i=1,2, \cdots, n$ respectivamente, tais que com $N \geq 2$,

$$
\rho_{i} \leq \rho_{f}:=1+\frac{2 q}{N} \text { e } \overline{\rho_{i}} \leq \rho_{g}:=1+\frac{q}{N}, i=1,2, \cdots n,
$$

sendo estrita a segunda desigualdade para o caso $N=1$.

Temos assim o 
Teorema 1.10 Suponhamos que $f_{i}, g_{i}, i=1,2, \cdots, n$ satisfaçam (C1). Então para todo $u_{0} \in L^{q}\left(\Omega, \mathbb{R}^{n}\right)$ o problema (1.1) possui uma única soluçāo $\varepsilon$-regular, $u\left(\cdot ; u_{0}\right)$, no sentido da Definição 1.12 , tal que $u\left(0 ; u_{0}\right)=u_{0}$, e dependendo continuamente de $u_{0} \in L^{q}\left(\Omega, \mathbb{R}^{n}\right)$. Além disso, esta solução é clássica.

Demonstração: Para uma prova detalhada deste teorema veja [5].

\subsubsection{Existência e Unicidade de Soluções em $W^{1, q}\left(\Omega, \operatorname{IR}^{n}\right)$}

Nesta subseção mostraremos que o problema (1.1) possui uma única solução em $W^{1, q}\left(\Omega, \mathbb{R}^{n}\right)$. Para isso, precisamos assumir que as não linearidades em (1.1) satisfaçam as seguintes condiçoes de crescimentos:

(C2) Suponhamos que para $i=1,2, \cdots, n f_{i}, g_{i}$ sejam funções localmente lipschitzianas tais que

1. $q>N$,

2. $q=N$ e para todo $\eta>0$, existam constantes $c_{\eta}>0$ tais que $f_{i}$ e $g_{i}$, $i=1,2, \cdots n$ satisfaçam uma relação da forma

$$
|h(u)-h(v)| \leq c_{\eta}|u-v|\left(e^{\eta|u|^{\frac{N}{N-1}}}+e^{\eta|v| \frac{N}{N-1}}\right), u, v \in \mathbb{R}^{n},
$$

3. $1<q<N$ e $f_{i}$ e $g_{i}, i=1,2, \cdots n$ satisfaçam a relação acima com expoentes $\rho_{i}$ e $\overline{\rho_{i}}, i=1,2, \cdots n$ respectivamente, tais que,

$$
\rho_{i} \leq \rho_{f}:=1+\frac{2 q}{N-q} \text { e } \overline{\rho_{i}} \leq \rho_{g}:=1+\frac{q}{N-q}, i=1,2, \cdots n .
$$

Com as notaçoes da Subseção 1.1.7, definamos

$$
X_{q}^{\alpha}:=E_{q}^{\alpha-\frac{1}{2}}, \quad \alpha \in \mathbb{R}
$$

e como em (1.51),

$$
A_{\alpha}: X_{q}^{1} \subset X_{q}^{0} \rightarrow X_{q}^{0},
$$

a $E_{q}^{-\frac{1}{2}}$-realizaçāo do operador $A$, como definido na Subseção 1.1.7. Com essas notações, (1.56) implica que 


$$
\begin{array}{lll}
X_{q}^{\alpha} \hookrightarrow L^{r}\left(\Omega, \mathbb{R}^{n}\right), & r \leq \frac{N q}{N+q-2 \alpha q}, & \frac{1}{2} \leq \alpha<\frac{N}{2 q}+\frac{1}{2} \\
X_{q}^{0}=W^{-1, q}\left(\Omega, \mathbb{R}^{n}\right) & \\
X_{q}^{\alpha} \hookleftarrow L^{s}\left(\Omega, \mathbb{R}^{n}\right), & s \geq \frac{N q}{N+q-2 \alpha q}, \quad \frac{1}{2}-\frac{N}{2 q^{\prime}}<\alpha \leq \frac{1}{2},
\end{array}
$$

onde $1<q<\infty$, com imersões contínuas.

Assim, seguindo os passos da Subseção anterior, provamos o próximo teorema, cuja prova pode ser encontrada com detalhes em [5].

Teorema 1.11 Suponhamos que $f_{i}, g_{i}, i=1,2, \cdots, n$ satisfaçam (C2). Então para todo $u_{0} \in W^{1, q}\left(\Omega, \mathbb{R}^{n}\right)$ o problema $(1.1)$ possui uma única solução $\varepsilon$-regular, $u\left(\cdot ; u_{0}\right)$, no sentido da Definição 1.11 , tal que $u\left(0 ; u_{0}\right)=u_{0}$, e dependendo continuamente de $u_{0} \in W^{1, q}\left(\Omega, \mathbb{R}^{n}\right)$. Além disso, esta solução é clássica.

Demonstraçāo: A prova deste teorema pode ser encontrada em [5]. 


\section{Capítulo 2}

\section{Existência Global de Soluções e Existência de Atratores Globais}

Neste capítulo, vamos mostrar que a solução encontrada no Capítulo 1, para o problema (1.1) é globalmente definida. Dividiremos esse trabalho em duas partes, primeiro consideraremos o caso da dimensão do sistema, $n=2$, e $d_{i}=1, i=1,2 \mathrm{em}$ (1.2). Depois consideraremos o caso $n \in \mathbb{N}$, com $k=0$ em (1.1) e $d_{i}>0, i=1,2, \cdots, n$ em (1.2). Ainda mais, em cada caso, mostraremos a existência de atratores globais para o referido problema, bem como algumas propriedades sobre o comportamento assintótico das soluções. Para fazermos isso, apresentaremos alguns resultados abstratos sobre comparação e positividade de soluções de equaçōes parabólicas semilineares, contidos em [6], depois adaptaremos alguns resultados contidos em [12] para obtermos comparação e positividade de soluções de problemas mais gerais que o problema (1.1) e que os problemas considerados em [6], o que nos permitirá mostrarmos a existência global de soluções bem como a existência de atratores globais desejadas.

\subsection{Resultados de Comparação e Positividade}

Nesta seção, mostraremos alguns resultados abstratos sobre comparação e positividade, depois aplicaremos tais resultados para operadores específicos, os quais nos serão úteis para mostrarmos a existência global, propriedades assintóticas e sincronização de soluções do problema (1.1). Os resultados abstratos de comparação e positividade de soluçōes desta seção estão contidos em [6]. 
Definição 2.1 Um espaço de Banach ordenado é um par $(X, \leq)$, onde $X$ é um espaço de Banach e $\leq$ é uma relação de ordem em $X$ tal que

- $x \leq y$ implica que $x+z \leq y+z$, para todo $x, y, z \in X$;

- $x \leq y$ implica que $\lambda x \leq \lambda y$ para $\lambda \in \mathbb{R}^{+}$e $x, y \in X$;

- $O$ "cone positivo" $C=\{x \in X ; 0 \leq x\}$ é fechado em $X$.

Notemos que $x \leq y$ é equivalente a $0 \leq y-x$. Também observemos que $x \leq 0 \mathrm{se}, \mathrm{e}$ somente se, $0 \leq-x$, e que o cone fechado $C$ é convexo.

Exemplo 2.1 Sejam $X=L^{q}(\Omega)$ ou $X=W^{1, q}(\Omega)$, munidos da ordem usual, isto é, se $f_{1}, f_{2} \in X$, entäo $f_{1} \leq f_{2}$ se, e somente se, $f_{1}(x) \leq f_{2}(x)$, para quase todo $x \in \Omega$. Assim, $(X, \leq)$ é um espaço de Banach ordenado.

Definição 2.2 Seja $(X, \leq)$ um espaço de Banach ordenado. Diremos que a aplicação $T: X \rightarrow X$ é crescente se

$$
x \leq y \Rightarrow T(x) \leq T(y), \quad x, y \in X,
$$

diremos que ela é positiva se

$$
0 \leq x \Rightarrow 0 \leq T(x), x \in X .
$$

Observemos que se $T$ na definição acima for linear, ambos os conceitos coincidem.

Lema 2.1 Sejam $(X, \leq)$ um espaço de Banach ordenado e $f \in L^{1}\left(\left(t_{0}, t_{1}\right), X\right)$, tal que $0 \leq f(t)$, para quase todo $t \in\left(t_{0}, t_{1}\right)$. Então

$$
0 \leq \int_{t_{0}}^{t_{1}} f(s) d s
$$

ou seja,

$$
\int_{t_{0}}^{t_{1}}: L^{1}\left(\left(t_{0}, t_{1}\right), X\right) \rightarrow X,
$$

é uma aplicação linear crescente. 
Demonstração: Como a integral é um operador linear e limitado entre $L^{1}\left(\left(t_{0}, t_{1}\right), X\right)$ e $X$ e, o cone positivo $C$ na Definição 2.1 é fechado, basta mostrarmos o resultado para $f$ em um subconjunto denso de $L^{1}\left(\left(t_{0}, t_{1}\right), X\right)$. Suponhamos então que $f \in C\left(\left[t_{0}, t_{1}\right], X\right)$. $\mathrm{O}$ Teorema do Valor Médio para integrais implica que

$$
\int_{t_{0}}^{t_{1}} f(s) d s \in\left(t_{1}-t_{0}\right) \overline{c o}\left(f\left(\left[t_{0}, t_{1}\right]\right)\right)
$$

onde $\overline{c o}\left(f\left(\left[t_{0}, t_{1}\right]\right)\right)$ é o fecho da envoltória convexa da imagem de $\left[t_{0}, t_{1}\right]$ por $f$. Se $0 \leq f(t)$, para todo $t \in\left(t_{0}, t_{1}\right)$, então esta imagem está em $C$, o cone da Definição 2.1. Assim,

$$
\overline{c o}\left(f\left(\left[t_{0}, t_{1}\right]\right)\right) \subset C,
$$

já que $C$ é convexo, o que mostra o resultado.

Sejam $(X, \leq)$ um espaço de Banach ordenado e $A: D(A) \subset X \rightarrow X$ um operador setorial. Consideremos $\lambda_{0} \in \mathbb{R}$ tal que

$$
\operatorname{Re}\left(\sigma\left(A+\lambda_{0}\right)\right)>0 .
$$

Suponhamos ainda que $A$ satisfaça a seguinte definição:

Definição 2.3 Um operador setorial $A$ em um espaço ordenado de Banach $(X, \leq)$ é dito ser resolvente positivo em $X$, se para todo $\lambda>\lambda_{0},(A+\lambda)^{-1}$ é um operador crescente em $X$.

O próximo resultado estabelece uma relação entre a positividade de um operador setorial e de seu semigrupo associado.

Proposição 2.1 Sejam A um operador setorial satisfazendo as condições acima $e$ $\left\{e^{-A t}, t \geq 0\right\}$ o semigrupo analítido gerado por $-A$. Então, $A$ é resolvente positivo se, e somente se, $e^{-. A t}$ é positivo, para todo $t \geq 0$.

Demonstração: Suponhamos inicialmente que $\lambda_{0}=0$, isto é $\operatorname{Re}(\sigma(A))>0$, e que para todo $\lambda>0$. $(A+\lambda)^{-1}$ seja um operador crescente. Para todos $t>0$ e $u_{0} \in X$, temos

$$
\left(I+\frac{t}{n} A\right)^{-n} u_{0} \rightarrow e^{-A t} u_{0}, \text { quando } n \rightarrow \infty
$$


veja Seção 1.8 de [21]. Em particular, se $0 \leq u_{0}$, então

$$
\left(I+\frac{t}{n} A\right)^{-n} u_{0}=\frac{t^{n}}{n^{n}}\left(\frac{n}{t}+A\right)^{-n} u_{0} \geq 0
$$

para todo $n \in \mathbb{N}$ e como o cone positivo $C$ é fechado, obtemos que $e^{-A t} u_{0} \geq 0$.

Para $\lambda \in \mathbb{R}$ arbitrário, como

$$
(A+\lambda)^{-1}=\left(A+\lambda_{0}+\left(\lambda-\lambda_{0}\right)\right)^{-1},
$$

tomando $A_{0}=A+\lambda_{0}$, temos $\left(A_{0}+s I\right)^{-1} \geq 0$, para todo $s>0$ e, entāo

$$
0 \leq e^{-A_{0} t}=e^{-\left(\lambda-\lambda_{0}\right) t} e^{-A t},
$$

o que mostra a primeira parte da proposição .

A recíproca segue do fato que

$$
(A+\lambda)^{-1}=\int_{0}^{\infty} e^{-A t} e^{-\lambda t} d t
$$

e do Lema 2.1.

Observação 2.1 Notemos que dizer que $A$ é resolvente positivo, é equivalente ao seguinte critério de comparação: para todo $\lambda>\lambda_{0}$ ef $\leq g$ em $X$, se

$$
A u+\lambda u=f \quad \text { e } \quad A v+\lambda v=g,
$$

então, $u \leq v$ em $X$.

Consideremos o problema

$$
\left\{\begin{array}{l}
u_{t}+A u=f(t), t \in\left(t_{0}, t_{1}\right) \\
u\left(t_{0}\right)=u_{0} \in X
\end{array}\right.
$$

em $(X, \leq)$ um espaço de Banach ordenado, onde $A$ é um operador setorial de resolvente positivo, satisfazendo as condições acima. Denotaremos por $u\left(t ; u_{0}, f\right)$ uma solução de (2.1), com dado inicial $u_{0}$, no intervalo $\left(t_{0}, t_{1}\right)$, Temos o

Corolário 2.1 Sejam $(X, \leq)$ um espaço de Banach ordenado e A um operador setorial de resolvente positivo, satisfazendo as condições acima. Suponhamos que $u_{1}, u_{2} \in X$ são tais que $u_{1} \leq x_{2}$ e $f_{1}(t) \leq f_{2}(t)$ para quase todo $t \in\left(t_{0}, t_{1}\right)$. Então

$$
u\left(t ; u_{1}, f_{1}\right) \leq u\left(t ; u_{2}, f_{2}\right),
$$

para quase todo $t \in\left(t_{0}, t_{1}\right)$. 
Demonstração: Lembrando que para $i=1,2$ a solução, $u\left(t ; u_{i}, f_{i}\right)$, de $(2.1)$ é dada pela fórmula da variação das constantes

$$
u\left(t ; u_{i}, f\right)=e^{-A\left(t-t_{0}\right)} u_{i}+\int_{t_{0}}^{t} e^{-A(t-s)} f_{i}(s) d s,
$$

$i=0,1$. O Lema 2.1 e a Proposição 2.1 demonstram o corolário.

Finalmente, consideremos problemas da forma

$$
\left\{\begin{array}{l}
u_{t}+A u=f(u), t>t_{0}, \\
u\left(t_{0}\right)=u_{0} \in X
\end{array}\right.
$$

onde $A$ e $X$ satisfazem as condições desta seção. Suponhamos que tenhamos construída, como na Seção 1.1, uma escala de espaços de interpolação, a qual denotaremos nesta seção por $X^{\alpha}, \alpha \geq 0, \operatorname{com} X^{0}=X$ e $X^{1}=D(A)$. Em cada $X^{\alpha}$, consideremos a ordem induzida por $X$. Durante esta seção, a escala $X^{\alpha}, \alpha \geq 0$ satisfará a seguinte definição :

Definição 2.4 A escala de espaços de Banach $X^{\alpha}, \alpha \geq 0$ é uma Escala de Espaços Ordenada se as inclusões $X^{\alpha} \hookrightarrow X^{\beta}, \alpha \geq \beta$, são positivas, $e$ as $\alpha$-realizações de $A$ em $X^{\alpha}, A_{\alpha}: X^{\alpha+1} \rightarrow X^{\alpha}, \alpha \geq 0$, são resolventes positivos e o cone positivo de $X^{\alpha}$ é denso no cone positivo de $X^{\beta}$ para todo $\alpha \geq \beta \geq 0$.

Suponhamos que o problema (2.2) seja localmente bem posto em $X^{1}$, isto é, para cada $u_{0} \in X^{1}$, existe uma única solução de $(2.2)$, a qual denotaremos por $u\left(t ; u_{0}, f\right)$, tal que $u\left(t ; u_{0}, f\right) \in C\left(\left[t_{0}, \tau\right), X^{1}\right)$ para algum $\tau=\tau\left(u_{0}\right)$, e que $u\left(t ; u_{0}, f\right)$ depende continuamente do dado inical $u_{0} \in X^{1}$.

Precisamos assumir também que o termo não linear em (2.2), $f$, é subcrítico em um certo espaço $X^{1+\varepsilon}, \varepsilon \geq 0$, isto é, existe $\gamma>0$ com $0 \leq 1+\varepsilon-\gamma<1$ tal que $f: X^{1+\varepsilon} \rightarrow X^{\gamma}$ é localmente lipschitziana. Recordemos que neste caso, se $u_{0} \in X^{1+\varepsilon}$, $u\left(t ; u_{0}, f\right)$ é dada pelo ponto fixo da fórmula da variação das constantes

$$
\mathcal{F}(u)(t):=e^{-A\left(t-t_{0}\right)} u_{0}+\int_{t_{0}}^{t} e^{-A(t-s)} f(u(s)) d s,
$$

no espaço $V=\left\{u\left(\cdot ; u_{0}, f\right) \in C\left(\left[t_{0}, t_{0}+\tau\right], X^{1+\varepsilon}\right) ;\left\|u\left(t ; u_{0}, f\right)\right\|_{X^{1}} \leq\left\|u_{0}\right\|_{X^{1+\varepsilon}}+\delta\right\}$, para certos $\tau, \delta$.

Temos então o seguinte resultado de positividade. 
Teorema 2.1 Sobre as hipóteses acima, suponhamos que para todo $r>0$ existe uma constante $\beta=\beta(r)>0$ tal que $f(\cdot)+\beta I$ é positiva nos elementos da bola de raio $r$ em $X^{1+\varepsilon}$. Se $0 \leq u_{0} \in X^{1}$, então a solução, $u\left(\cdot ; u_{0}, f\right)$, de (2.2) é positiva enquanto ela existir.

Demonstração: Suponhamos primeiro que $u_{0} \in X^{1+\varepsilon}$. Para $\beta>0$, consideremos

$$
\mathcal{F}(u)(t):=e^{-(A+\beta I)\left(t-t_{0}\right)} u_{0}+\int_{t_{0}}^{t} e^{-(A+\beta I)(t-s)}(f(u(s))+\beta(u(s))) d s .
$$

Da mesma forma que na Seção 1.2, mostramos que $\mathcal{F}$ é uma contração em

$$
V:=\left\{u \in C\left(\left[t_{0}, t_{0}+h\right], X^{1+\varepsilon}\right) ;\left\|u(t)-u_{0}\right\|_{X^{1+\varepsilon}} \leq \delta, t \in\left[t_{0}, t_{0}+h\right]\right\},
$$

se $h, \delta>0$, são pequenos o suficiente.

Tomando $\beta=\beta\left(\left\|u_{0}\right\|_{X^{1+\varepsilon}}+\delta\right)$, como $A$ é resolvente positivo, então

$$
e^{-(A+\beta I)} u \geq 0 \text { e } f(u)+\beta u \geq 0,
$$

para todo $0 \leq u \in B_{X^{1+\varepsilon}}\left(0,\left\|u_{0}\right\|_{X^{1+\varepsilon}}+\delta\right)$. Se $u_{0} \geq 0$, a definição de $\mathcal{F}$ implica que

$$
\mathcal{F}(u) \geq 0,
$$

para todo $0 \leq u \in B_{X^{1+\varepsilon}}\left(0,\left\|u_{0}\right\|_{X^{1+\varepsilon}}+\delta\right)$. Portanto, se denotarmos por $V_{+}:=\{u \in$ $V \mid u \geq 0\}$, então

$$
\mathcal{F}\left(V_{+}\right) \subset V_{+}
$$

Assim, o único ponto fixo de $\mathcal{F}$ em $V$ pertence à $V_{+}$. Logo $u(t) \geq 0$, para todo $t \in$ $\left[t_{0}, t_{0}+h\right]$, e um argumento de continuação mostra que $u(t) \geq 0$, enquanto a solução estiver definida.

Suponhamos agora que $0 \leq u_{0} \in X^{1}$. Como o cone positivo de $X^{1+\varepsilon}$ é denso no cone positivo de $X^{1}$, existe uma sequência $0 \leq u_{0}^{n} \in X^{1+\epsilon}, n \geq 1$ tal que

$$
u_{0}^{n} \rightarrow u_{0}, \text { quando } n \rightarrow \infty .
$$

O caso anterior implica que $u^{n}\left(t ; u_{n}\right) \geq 0$, em $X^{1+\varepsilon}$, para cada $n \geq 1$. A dependência contínua em relação aos dados iniciais implica que

$$
u^{n}\left(t ; u_{0}^{n}\right) \rightarrow u\left(t ; u_{0}\right), \text { quando } n \rightarrow \infty,
$$

em $X^{1+\varepsilon}$. Como o cone em $X^{1+\varepsilon}$ é fechado, o resultado segue. 
Observação 2.2 Notemos que se $f$ é uma aplicação $\varepsilon$-regular em relação ao par $\left(X^{1}, X^{0}\right)$, isto é, $f: X^{1+\varepsilon} \rightarrow X^{\gamma(\varepsilon)}$, para algum $\rho>1$ tal que $\rho \varepsilon \leq \gamma(\varepsilon)$, e satisfaz

$$
\|f(x)\|_{X^{\gamma(\varepsilon)}} \leq C\left(\|x\|_{X^{1+\varepsilon}}^{\rho}+1\right) .
$$

Então $f$ é localmente Lipschitz em subconjuntos limitados de $X^{1+\varepsilon}$.

Com o mesmo raciocínio do Teorema 2.1 podemos mostrar o seguinte resultado sobre comparação de soluções:

Teorema 2.2 Sejam A e $X$ como acima, e suponhamos que as não linearidades $f, g$ e h satisfaçam as condições descritas acima para $f$. Então

(i) Suponhamos que para todo $r>0$ exista uma constante $\beta=\beta(r)>0$ tal que $f(\cdot)+\beta I$ seja crescente na bola de raio $r$ de $X^{1+\varepsilon}$. Se $u_{0}, u_{1} \in X^{1}$, com $u_{0} \leq u_{1}$ então, $u\left(\cdot ; u_{0}, f\right) \leq u\left(\cdot ; u_{1}, f\right)$ enquanto elas existirem.

(ii) Suponhamos que $f$ e $g$ satisfaçam $f(\cdot) \leq g(\cdot)$. Então, para todo $u_{0} \in X^{1}$, $u\left(\cdot ; u_{0}, f\right) \leq u\left(\cdot ; u_{0}, g\right)$ enquanto elas existirem.

(iii) Suponhamos que $f, g$ sejam tais que para todo $r>0$, exista uma constante $\beta=$ $\beta(r)>0$ e uma função crescente, $h$, tal que $f+\beta I \leq h \leq g+\beta I$ na bola de raio $r$ em $X^{1+\varepsilon}$. Se $u_{0}, u_{1} \in X^{1}$ com $u_{0} \leq u_{1}$ então, $u\left(\cdot ; u_{0}, f\right) \leq u\left(\cdot ; u_{0}, g\right)$ enquanto elas existirem.

Demonstração: Como no Teorema 2.1, basta mostrarmos os resultados para $u_{0} \in$ $X^{1+\varepsilon}$.

Para mostramos (i), sejam $u_{i}(t):=u\left(t ; u_{i}, f\right), i=0,1$. Sabemos que para $i=0,1$, $u_{i}$ são os únicos pontos fixos da aplicação

$$
\mathcal{F}(u)(t):=e^{-(A+\beta I)\left(t-t_{0}\right)} u_{i}+\int_{t_{0}}^{t} e^{-(A+\beta I)(t-s)}(f(u(s))+\beta(u(s))) d s,
$$

no espaço

$$
V_{i}:=\left\{u \in C\left(\left[t_{0}, t_{0}+h\right], X^{1+\varepsilon}\right) ;\left\|u(t)-u_{i}\right\|_{X^{1+\varepsilon}} \leq \delta, \quad t \in\left[t_{0}, t_{0}+h\right]\right\},
$$

para $\delta, h>0$ suficientemente pequenos.

Considerando

$$
\tilde{V}_{1}:=\left\{u \in V_{0} \mid u(t) \geq u_{0}(t), \quad t \in\left[t_{0}, t_{0}+h\right]\right\}
$$


que é não vazio pois $u(t)=u_{0}(t)+u_{1}-u_{0}$ é um elemento de $\tilde{V}_{1}$, temos que

$$
\mathcal{F}\left(\tilde{V}_{1}\right) \subset \tilde{V}_{1}
$$

Mas isto implica que o único ponto fixo de $\mathcal{F}$ em $V_{1}$ é tal que $u_{1}(t) \geq u_{0}(t)$, para $t \in\left[t_{0}, t_{0}+h\right]$. E um argumento padrão de continuação demonstra o item (i).

Com um raciocínio semelhante mostramos o item (ii). Para o item (iii), temos pelo item (ii) que $u\left(t ; u_{0}, f\right) \leq u\left(t ; u_{0}, h\right)$ e $u\left(t ; u_{1}, h\right) \leq u\left(t ; u_{1}, g\right)$ enquanto elas existirem. Finalmente $o$ item (i) demonstra o teorema.

A idéia agora é aplicarmos esses resultados para obtermos alguns resultados sobre positividade e comparação para soluções do problema (1.1). Na realidade, obteremos resultados de comparação e positividade para problemas um pouco mais gerais do que o problema (1.1). Como iremos trabalhar com sistemas, precisamos definir uma ordem em $X=L^{q}\left(\Omega, \mathbb{R}^{n}\right)$ ou $X=W^{1, q}\left(\Omega, \mathbb{R}^{n}\right)$, para cada $n \in \mathbb{N}$. Temos o

Exemplo 2.2 Definamos uma ordem em $X=L^{q}\left(\Omega, \mathbb{R}^{n}\right)$ ou $X=W^{1, q}\left(\Omega, \mathbb{R}^{n}\right)$, $n \in \mathbb{N}$ e $1<q<\infty$, a qual denotaremos por $\preceq$, da seguinte maneira: sejam $f=$ $\left(f_{1}, f_{2}, \cdots, f_{n}\right), g=\left(g_{1}, g_{2}, \cdots, g_{n}\right) \in X$, diremos que $f \preceq g$ se $f_{i} \leq g_{i}, i=1,2, \cdots, n$, onde $\leq$ é a ordem usual de $L^{q}(\Omega)$ ou $W^{1, q}(\Omega)$, respectivamente. Claramente $(X, \preceq)$ é um espaço de Banach ordenado.

Os dois próximos resultados são adaptações de resultados contidos em [12], e nos serão úteis para mostrarmos que os operadores associados à problemas do tipo (1.1) são resolventes positivos em $L^{q}\left(\Omega, \mathbb{R}^{n}\right)$.

Lema 2.2 Sejam $H$ um espaço de Hilbert ef $f \in H$. Se existir $\tilde{f} \in H$ tal que

$$
\|\tilde{f}\| \leq\|f\| e\langle\tilde{f}, f\rangle \geq|\langle f, f\rangle|,
$$

onde $\langle\cdot, \cdot\rangle$ denota o produto interno em $H$, então $\tilde{f}=f$.

Demonstração: Notemos primeiro que para toda $f \in H$, temos

$$
\|f\|^{2}=|\langle f, f\rangle| \leq\langle\bar{f}, f\rangle \leq\|\bar{f}\|\|f\| .
$$

Logo, $\|\tilde{f}\|=\|f\|$. Assim,

$$
0 \leq\langle f-\tilde{f}, f-\tilde{f}\rangle=2\|f\|^{2}-2\langle\tilde{f}, f\rangle \leq 0
$$


e, portanto, $f=\tilde{f}$.

Observemos que para $X=L^{2}\left(\Omega, \mathbb{R}^{n}\right)$, tomando $\tilde{f}=|f|$, onde $|f|=\left(\left|f_{1}\right|, \cdots,\left|f_{n}\right|\right)$, para toda $f \in L^{2}\left(\Omega, \mathbb{R}^{n}\right)$, as condições do Lema 2.2 estão sempre satisfeitas, O próximo resultado nos dá uma condição suficiente para um operador, $A$ definido em $H$, ser resolvente positivo em $H$, conforme a Definição 2.3.

Teorema 2.3 Sejam $(H, \leq)$ um espaço de Hilbert ordenado, $C$ o seu cone positivo $e$ $A: D(A) \subset H \rightarrow H$ um operador autoadjunto e positivo, isto é, $(A u, u\rangle \geq 0$, para todo $u \in D(A)$. Suponhamos que exista um subconjunto denso $\mathcal{D} \subset H$ tal que:

1. $(A+\alpha)^{-1} \mathcal{D} \subset \mathcal{D}$, para todo $\alpha \geq 0$;

2. para cada $d \in \mathcal{D}$, podemos definir $|d| \in \mathcal{D} \cap C$ tal que $\|d\|=\||d|\|$. Ainda, se $d \in \mathcal{D}$, então $d \in C$ se, e somente, se $d=|d|$;

3. para todo $d \in \mathcal{D}$ e $g \in C,\langle|d|, g\rangle \geq,|\langle d, g)|$;

4. se $u \in D\left(A^{1 / 2}\right)$, então $|u| \in D\left(A^{1 / 2}\right) e$

$$
\left\langle A^{1 / 2}|u|, A^{1 / 2}|u|\right\rangle \leq\left(A^{1 / 2} u, A^{1 / 2} u\right\rangle .
$$

Então, $A$ é resolvente positivo em $H$.

Demonstração: Consideremos em $D\left(A^{\frac{1}{2}}\right)$ o produto interno dado por

$$
\langle f, g\rangle_{1}=\left\langle A^{\frac{1}{2}} f, A^{\frac{1}{2}} g\right\rangle+\alpha\langle f, g\rangle,
$$

para $f, g \in D\left(A^{\frac{1}{2}}\right)$ e para todo $\alpha>0$. Seja $X^{\frac{1}{2}}$ o espaço de Hilbert definido por

$$
X^{\frac{1}{2}}:=\left(D\left(A^{\frac{1}{2}}\right),\langle\cdot, \cdot\rangle_{1}\right)
$$

Sejam $g \in \mathcal{D}$ tal que $g \in C$ e $c=(A+\alpha)^{-1} g$. Então, como $A^{\frac{1}{2}}$ é autoadjunto, temos que

$$
\begin{aligned}
\langle|c|, c\rangle_{1} & =\left\langle|c|,(A+\alpha)^{-1} g\right\rangle_{1}=\left\langle A^{\frac{1}{2}}|c|, A^{\frac{1}{2}}(A+\alpha)^{-1} g\right\rangle+\alpha\left\langle|c|,(A+\alpha)^{-1} g\right) \\
& =\left\langle(A+\alpha)|c|,(A+\alpha)^{-1} g\right\rangle=\langle|c|, g\rangle \geq|\langle c, g\rangle| .
\end{aligned}
$$


Ainda mais,

$$
\begin{aligned}
\||c|\|_{1}^{2} & =\left\langle A^{\frac{1}{2}}|c|, A^{\frac{1}{2}}|c|\right\rangle+\||c|\|^{2} \\
& \leq\left\langle A^{\frac{1}{2}} c, A^{\frac{1}{2}} c\right\rangle+\alpha\|c\|^{2} \\
& =\|c\|_{1}^{2} .
\end{aligned}
$$

Usando o Lema $2.2 \operatorname{com} f=c$ e $\tilde{f}=|c|$, concluímos que se $g \in \mathcal{D} \cap C$, então

$$
\left|(A+\alpha)^{-1} g\right|=|c|=c=(A+\alpha)^{-1} g
$$

e, portanto $(A+\alpha)^{-1} g \in C$.

Agora, a densidade de $\mathcal{D}$ em $H$ e a continuidade de $(A+\alpha)^{-1}$, implicam que

$$
(A+\alpha)^{-1} g \in C, \text { para todo } g \in C,
$$

mostrando que $A$ é resolvente positivo em $H$.

Consideremos, agora, para todo $n \geq 1$ e $1<q<\infty$, o operador $\tilde{B}$ definido por $\tilde{B}: D(\tilde{B}) \subset L^{q}\left(\Omega, \mathbb{R}^{n}\right) \rightarrow L^{q}\left(\Omega, \mathbb{R}^{n}\right)$ com

$$
\begin{aligned}
& D(\tilde{B})=\left\{\phi \in W^{2, q}\left(\Omega, \mathbb{R}^{n}\right) ; D \frac{\partial \phi}{\partial \tilde{n}}=-K_{n} \phi \text { em } \Gamma\right\} \\
& \tilde{B} \phi_{i}=-D \triangle \phi+c \phi
\end{aligned}
$$

para $\phi \in D(\tilde{B})$, onde $K_{n}:=\left\{k_{i j}\right\}_{n \times n}$, a qual será chamada de matriz de acoplamento, 'e uma matriz simétrica de ordem $n$, tal que para todo $u \in \mathbb{R}^{n}$,

$$
K_{n} u \cdot u \geq 0
$$

e

$$
K_{n}|u| \cdot|u| \leq K_{n} u \cdot u
$$

onde $\left|\left(u_{1}, \cdots, u_{n}\right)\right|=\left(\left|u_{1}\right|, \cdots,\left|u_{n}\right|\right)$, " " é o produto interno usual em $\mathbb{R}^{n}, D$ é a seguinte matriz $n \times n$ :

$$
D:=\left[\begin{array}{lllll}
d_{1} & 0 & 0 & \cdots & 0 \\
0 & d_{2} & 0 & \cdots & 0 \\
0 & 0 & d_{3} & \cdots & 0 \\
\vdots & \vdots & \vdots & \ddots & \vdots \\
0 & 0 & 0 & \cdots & d_{n}
\end{array}\right]
$$


com $d_{i} \geq 1$ para $i=1,2, \cdots, n$ e $c \geq 0$ é uma constante tomada de tal forma que o primeiro autovalor, $\mu_{1}(K)$, associado ao operador $\tilde{B}$ seja positivo. Temos o seguinte resultado:

Proposição 2.2 O operador $\tilde{B}$ definido em (2.3) é um operador setorial, e é resolvente positivo em $L^{q}\left(\Omega, \mathbb{R}^{n}\right)$, para todo $n \geq 1$.

Demonstração: Primeiramente, vamos usar o Teorema 2.3 para mostrarmos que $\tilde{B}$ é resolvente positivo em $L^{2}\left(\Omega, \mathbb{R}^{n}\right)$. Para isso, vamos mostrar que todas as condições do Teorema 2.3 estão satisfeitas.

Para todo $\phi \in D(\tilde{B})$, integrando por partes, obtemos que

$$
\begin{aligned}
\langle\tilde{B} \phi, \phi\rangle_{L^{2}\left(\Omega, \mathbf{R}^{n}\right)} & =\langle-D \Delta \phi, \phi\rangle_{L^{2}\left(\Omega, \mathbb{R}^{n}\right)}+c\langle\phi, \phi\rangle_{L^{2}\left(\Omega, \mathbb{R}^{n}\right)} \\
& =\left\langle\int_{\Omega}|\nabla \phi(x)|^{2}+\int_{\partial \Omega} K_{n} \phi \cdot \phi+c\|\phi\|_{L^{2}\left(\Omega, \mathbb{R}^{n}\right)}^{2},\right.
\end{aligned}
$$

logo (2.4) mostra que $\tilde{B}$ é um operador positivo. A simetria da matriz $K_{n}$ e um cálculo semelhante ao feito acima mostra que

$$
\langle\tilde{B} \phi, \psi\rangle_{L^{2}\left(\Omega, \mathbb{R}^{n}\right)}=\langle\phi, \tilde{B} \psi\rangle_{L^{2}\left(\Omega, \mathbb{R}^{n}\right)},
$$

para todo $\psi, \phi \in \Delta(\tilde{B})$. Mas 0 está no conjunto resolvente de $\tilde{B}$, então conclúrmos que $\tilde{B}$ é autoadjunto.

Agora, consideremos o conjunto $\mathcal{D}$ como sendo o próprio $L^{2}\left(\Omega, \mathbb{R}^{n}\right)$. Assim, a condição 1 do Teorema 2.3 está claramente satisfeita. Para toda $f=\left(f_{1}, \cdots, f_{n}\right) \in$ $L^{2}\left(\Omega, \mathbb{R}^{n}\right)$, consideremos $|f|=\left(\left|f_{1}\right|, \cdots,\left|f_{n}\right|\right)$, onde para cada $i=1,2, \cdots, n,\left|f_{i}\right|(x)=$ $\left|f_{i}(x)\right|$, para todo $x \in \Omega$. Com a definição de ordem em $L^{2}\left(\Omega, \mathbb{R}^{n}\right)$, dada no Exemplo 2.2 , condição 2 segue imediatamente. A condição 3 é fácilmente verificada. Resta verificarmos a condição 4. Mas, para todo $\phi \in D(\tilde{B})$, integrando por partes e usando (2.5), temos

$$
\langle\tilde{B}|\phi|,|\phi|\rangle_{L^{2}\left(\Omega, \mathbb{R}^{n}\right)} \leq\langle\tilde{B} \phi, \phi\rangle_{L^{2}\left(\Omega, \mathbb{R}^{n}\right)}
$$

como $D(\tilde{B})$ está contido densamente em $D\left(\tilde{B}^{1 / 2}\right)$ e usando o fato que $\tilde{B}$ é um operador fechado, um argumento de densidade mostra a condição 4 .

Portanto, o Teorema 2.3 implica que $(\tilde{B}+\alpha)^{-1}$ é crescente para todo $\alpha>0$. 
Agora, para todo $q \geq 2$, temos que $L^{q}\left(\Omega, \mathbb{R}^{n}\right) \stackrel{d}{\hookrightarrow} L^{2}\left(\Omega, \mathbb{R}^{n}\right), \tilde{B}$ é resolvente positivo em $L^{2}\left(\Omega, \mathbb{R}^{n}\right)$ e o cone positivo de $L^{q}\left(\Omega, \mathbb{R}^{n}\right)$ está contido no cone positivo de $L^{2}\left(\Omega, \mathbb{R}^{n}\right)$. Assim, com um cálculo simples, obtemos que $\tilde{B}$ é resolvente positivo em $L^{q}\left(\Omega, \mathbb{R}^{n}\right)$.

Para $1<q \leq 2$, como cone positivo de $L^{2}\left(\Omega, \mathbb{R}^{n}\right)$ está densamene contido no cone positivo de $L^{q}\left(\Omega, \mathbb{R}^{n}\right), \tilde{B}$ é resolvente positivo em $L^{2}\left(\Omega, \mathbb{R}^{n}\right)$ e $(\tilde{B}+\alpha)^{-1}$ é contínuo em $L^{q}\left(\Omega, \mathbb{R}^{n}\right)$, um argumento de densidade mostra que $\tilde{B}$ é resolvente positivo em $L^{q}\left(\Omega, \mathbb{R}^{n}\right)$.

Como na Seção 1.1, podemos construir uma escala de Espaços de Banach, $X_{q}^{\alpha}$, $\alpha \in[-1,1], 1<q<\infty$, para o operador $\tilde{B}$ definido em (2.3), a Proposição 2.2, o Teorema 2.7.2 de [3] e o fato de $\mu_{1}(k)$ ser positivo, mostram o seguinte resultado:

Teorema 2.4 Para todo $1<q<\infty e \alpha \in[0,1], X_{q}^{\alpha}$ é um espaço ordenado com a ordem induzida por $L^{q}\left(\Omega, \mathbb{R}^{n}\right)$. O espaço $X_{q}^{-\alpha}$ é um espaço ordenado com a ordem dual canônica, isto é, $0 \leq u$ em $X_{q}^{-\alpha}$ se, e somente, se $0 \leq\langle u, \phi\rangle$ para todo $\phi \in X_{q^{\alpha}}^{\alpha}$.

Para $\gamma \geq \beta$, a inclusão $X_{q}^{\gamma} \hookrightarrow X_{q}^{\beta}$ é positiva e o cone positivo de $X_{q}^{\gamma}$ é denso no cone positivo de $X_{q}^{\beta}$.

Ainda, $B_{\alpha-1}: X_{q}^{\alpha} \rightarrow X_{q}^{\alpha-1}$ é um operador setorial e é resolvente positivo.

Com tudo isso, estamos aptos para obtermos resultados de comparação e positividade para soluções do seguinte problema parabólico semilinear, com acoplamento na fronteira:

$$
\left\{\begin{array}{l}
u_{t}(t, x)=D \Delta u(t, x)+F(u(t, x)) \quad t>0, \quad x \in \Omega, \\
D \frac{\partial u}{\partial \vec{n}^{(}}(t, x)=-K_{n} u(t, x)+G(u(t, x)) \quad t>0, \quad x \in \Gamma,
\end{array}\right.
$$

onde $u, k, \Omega, \Gamma$ são como definidos para (1.1), $D$ é a matriz $n \times n$ definida em (2.6) e $F=\left(F_{1}, F_{2}, \cdots, F_{n}\right): \mathbb{R}^{n} \rightarrow \mathbb{R}^{n}$ e $G=\left(G_{1}, G_{2}, \cdots, G_{n}\right): \mathbb{R}^{n} \rightarrow \mathbb{R}^{n}$ são duas funções tais que se $u=\left(u_{1}, u_{2}, \cdots, u_{n}\right)$, então

$$
\begin{aligned}
& F_{i}(u)=c u_{i}+f_{i}(u), \quad i=1,2, \cdots, n, \\
& G_{i}(u)=g_{i}(u), \quad i=1,2, \cdots, n,
\end{aligned}
$$

onde $c>0$ é como na definição do operador $\tilde{B}$ em (2.3). Temos assim o seguinte resultado de comparação e positividade: 
Proposição 2.3 Seja $\tilde{B}$ o operador definido em (2.3). Suponhamos que para $i=$ $0,1,2, \cdots, n, f_{i}, g_{i}: \mathbb{R}^{n} \rightarrow \mathbb{R}$ sejam funções localmente lipschitzianas satisfazendo as condições $(C 1)$ e $(C 2)$ definidas na Seção 1.3.

(i) Suponhamos que $f_{i}(0, \cdots, 0), g_{i}(0, \cdots, 0) \geq 0, i=1,2, \cdots, n$. Se $0 \preceq u_{0}$ então, $0 \preceq u\left(t ; u_{0}\right)$, enquanto ela existir.

(ii) Se $u_{0} \preceq u_{1}$. Então, $u\left(t ; u_{0}\right) \preceq u\left(t ; u_{1}\right)$, enquanto elas existirem.

(iii) Suponhamos que para todo $u \in \mathbb{R}^{n}, f_{0}^{i}(u) \leq f_{i}(u)$ e $g_{0}^{i}(u) \leq g_{i}(u)$, para $i=$ $1,2, \cdots, n$. Se $u_{0} \preceq u_{1}$ então, $u\left(t ; u_{0}, F_{0}, G_{0}\right) \preceq u\left(t ; u_{1}, F, G\right)$, enquanto elas existirem, onde $F_{0}=\left(f_{0}^{1}, \cdots, f_{0}^{n}\right)$ e $G_{0}=\left(g_{0}^{1}, \cdots, g_{0}^{n}\right), F$ e $G$ são definidas em (2.8).

Onde" $\preceq "$ denota a ordem definida no Exemplo 2.2, para o espaço onde o dado inicial $u_{i}, i=0,1$ pertença, isto é, $L^{q}\left(\Omega, \mathbb{R}^{n}\right)$ ou $W^{1, q}\left(\Omega, \mathbb{R}^{n}\right)$.

Demonstração: Provaremos esta proposição apenas para o caso $n=2$, o caso $n>2$ prova-se de maneira análoga. Suponhamos inicialmente que $u_{0}, u_{1} \in W^{1, s}\left(\Omega, \mathbb{R}^{2}\right)$ para $s>N$. Então existe $T>0$ tal que $u\left(t ; u_{0}, F_{0}, G_{0}\right)$ e $u\left(t ; u_{1}, F, G\right)$ estão definidas no intervalo fechado $[0, T]$ e tal que

$$
\xi:=\sup _{t \in[0, T]}\left\{\left\|u\left(t ; u_{0}, F_{0}, G_{0}\right)\right\|_{L^{\infty}\left(\Omega, \mathbb{R}^{2}\right)},\left\|u\left(t ; u_{1}, F, G\right)\right\|_{L^{\infty}\left(\Omega, \mathbb{R}^{2}\right)}\right\}<+\infty .
$$

Dessa forma, podemos assumir que $f_{0}^{1}, f_{0}^{2}, f_{1}, f_{2}, g_{1}$ e $g_{2}$ são funções globalmente lispchitzianas. Portanto, existe $\beta \in \mathbb{R}$ tal que para $i=1,2$

$$
\begin{array}{ll}
f_{0}^{i}(s, r)+\beta(s+r) & f_{i}(s, r)+\beta(s+r), \\
g_{0}^{i}(s, r)+\beta(s+r), & g_{i}(s, r)+\beta(s+r),
\end{array}
$$

são funções crescentes em $s \in \mathbb{R}$, para cada $r \in \mathbb{R}$ e crescentes em $r \in \mathbb{R}$, para cada $s \in \mathbb{R}$.

As conclusões da proposição para este caso, seguem agora da Proposição 2.2 e dos Teoremas 2.1 e 2.2, levando em conta a ordem definida no Exemplo 2.2.

Suponhamos agora que $u_{0}, u_{1} \in W^{1, q}\left(\Omega, \mathbb{R}^{2}\right)$, para $q \leq N$. Faremos a prova apenas para o caso (iii), os outros casos seguem de forma análoga. Consideremos sequências 
$\phi_{n}, \psi_{n} \in C_{0}^{\infty}\left(\Omega, \mathbb{R}^{2}\right), n \in \mathbb{N}$, tais que

$$
\begin{aligned}
& \phi_{n} \rightarrow u_{0}, \\
& \psi_{n} \rightarrow u_{1},
\end{aligned}
$$

em $W^{1, q}\left(\Omega, \mathbb{R}^{2}\right)$. Definamos, para cada $x \in \Omega$ e para $n \in \mathbb{N}$

$$
\begin{aligned}
& u_{0}^{n}(x):=\max \left\{\phi_{n}(x), \psi_{n}(x)\right\}, \\
& u_{1}^{n}(x):=\min \left\{\phi_{n}(x), \psi_{n}(x)\right\} .
\end{aligned}
$$

Assim,

$$
u_{0}^{n} \leq u_{1}^{n}, \forall n \in \mathbb{N}
$$

Logo, da parte anterior obtemos que

$$
u\left(t ; u_{0}^{n}, F_{0}, G_{0}\right) \leq u\left(t ; u_{1}^{n}, F, G\right),
$$

para todo $n \in \mathbb{N}$, em $W^{1, q}\left(\Omega, \mathbb{R}^{2}\right)$. Como as soluçōes dependem continuamente dos dados iniciais, segue que

$$
u\left(t ; u_{0}, F_{0}, G_{0}\right) \leq u\left(t ; u_{1}, F, G\right)
$$

em $W^{1, q}\left(\Omega, \mathbb{R}^{2}\right)$, concluíndo a prova de (iii).

\subsection{Existência Global e de Atratores Globais}

Nesta seçāo aplicaremos os resultados da Seção 2.1, para mostrarmos que as soluções locais do problema (1.1), encontradas nos Teoremas 1.10 e 1.11 são globalmente definidas. Mostraremos também a existência de atratores globais compactos para o referido problema, bem como algumas propriedades assintóticas de tais soluções. Seguiremos aqui as idéias contidas em [6]. 
Consideremos o seguinte problema

$$
\left\{\begin{array}{l}
u_{t}(t, x)=\Delta u(t, x)+f_{1}(u(t, x)) \quad t>0, \quad x \in \Omega, \\
v_{t}(t, x)=\Delta v(t, x)+f_{2}(v(t, x)) \quad t>0, \quad x \in \Omega, \\
\frac{\partial u}{\partial \vec{n}^{2}}(t, x)=g_{1}(u(t, x))-k(u(t, x)-v(t, x)) \quad t>0, \quad x \in \Gamma, \\
\frac{\partial v}{\partial \vec{n}^{2}}(t, x)=g_{2}(v(t, x))+k(u(t, x)-v(t, x)) \quad t>0, \quad x \in \Gamma .
\end{array}\right.
$$

onde $\Omega, \Gamma, k, f_{i}, g_{i}$ são como definidos para (1.1). Denotemos por $u\left(t ; u_{0}, f\right)$ uma solução de (2.9), com dado inicial $u_{0} \in L^{q}\left(\Omega, \mathbb{R}^{2}\right)$ ou $u_{0} \in W^{1, q}\left(\Omega, \mathbb{R}^{2}\right)$.

Para mostrarmos a existência global, precisamos assumir algumas restrições sobre o sinal dos termos não lineares de (2.9). Suponhamos que existam constantes $B_{0}, C_{0} \in \mathbb{R}$ e $B_{1}, C_{1} \geq 0$ tais que para todo $u \in \mathbb{R}$,

$$
\begin{aligned}
& u f_{i}(u) \leq-C_{0} u^{2}+C_{1}|u|, \\
& u g_{i}(u) \leq-B_{0} u^{2}+B_{1}|u|,
\end{aligned}
$$

$i=1,2$. Também, suponhamos que o primeiro autovalor, $\lambda_{1}$, do problema

$$
\left\{\begin{array}{l}
-\Delta u+C_{0} u=\lambda u \quad \text { em } \quad \Omega, \\
\frac{\partial u}{\partial \vec{n}}+B_{0} u=0 \quad \text { em } \quad \Gamma
\end{array}\right.
$$

seja positivo, onde $B_{0}, C_{0}$ são dados em (2.10). Com isso temos o seguinte resultado. Lema 2.3 Seja $\lambda_{1}(k)$ o primeiro autovalor do problema

$$
\left\{\begin{array}{l}
-\Delta u+C_{0} u=\lambda u \quad \text { em } \Omega \\
-\Delta v+C_{0} v=\lambda v \quad \text { em } \Omega \\
\frac{\partial u}{\partial \vec{n}}+B_{0} u+k(u-v)=0 \quad \text { em } \Gamma \\
\frac{\partial v}{\partial \vec{n}}+B_{0} v-k(u-v)=0 \quad \text { em } \Gamma
\end{array}\right.
$$

onde $B_{0}, C_{0}$ são dados em (2.10). Então, $\lambda_{1}(k)=\lambda_{1}>0$, para todo $k>0$. 
Demonstração: Considerando a mudança de variáveis $w=u-v$ e $z=u+v$, obtemos de (2.12) que $\lambda_{1}(k)$ é ainda o primeiro autovalor do problema

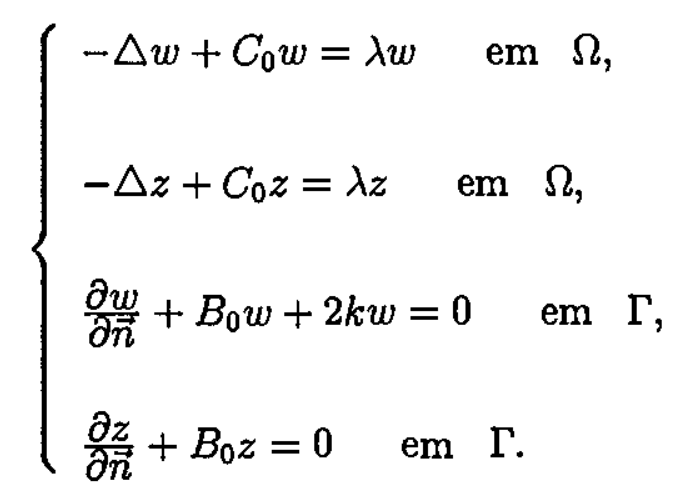

Como o problema acima é desacoplado, denotando por $\tilde{\mu}_{1}(k) \circ$ primeiro autovalor do problema

$$
\left\{\begin{array}{l}
-\Delta \phi+C_{0} \phi=\lambda \phi \quad \text { em } \quad \Omega \\
\frac{\partial \phi}{\partial \vec{n}}+B_{0} \phi+2 k \phi=0 \quad \text { em } \quad \Gamma
\end{array}\right.
$$

os resultados de [11], página 399, implicam que $\tilde{\mu}_{1}(k) \geq \lambda_{1}>0$, para todo $k \geq 0$, o que demonstra o lema.

Consideremos agora o operador $B$ definido por $B: D(B) \subset L^{q}\left(\Omega, \mathbb{R}^{2}\right) \rightarrow L^{q}\left(\Omega, \mathbb{R}^{2}\right)$ com

$$
\begin{aligned}
& D(B)=\left\{\phi \in W^{2, q}\left(\Omega, \mathbb{R}^{2}\right) ;-\frac{\partial \phi_{1}}{\partial \vec{n}}-b \phi_{1}=\frac{\partial \phi_{2}}{\partial \vec{n}}+b \phi_{2}=k\left(\phi_{1}-\phi_{2}\right) \text { em } \Gamma\right\} \\
& B \phi=-\Delta \phi+c \phi
\end{aligned}
$$

onde $c \geq 0$ é tomado de tal forma que o primeiro autovalor, $\mu_{1}(k)$, associado ao operador $B$ seja positivo. Notemos que o operador $B$ é um caso particular do operador $\tilde{B}$ definido em (2.3), então valem os resultados de comparação e positividade da Proposição 2.3 para soluções do problema (2.9). Também,

Observação 2.3 Observemos que com uma prova análoga ao do Lema 2.3, podemos tomar $c>0$, com $c$ independente de $k$, na definição do operador $B$, em (2.13), de tal 
forma que o primeiro autovalor, $\mu_{1}$, do problema

$$
\left\{\begin{array}{l}
-\Delta u+c u=\lambda u \quad \text { em } \Omega, \\
\frac{\partial u}{\partial \vec{n}}=0 \quad \text { em } \Gamma
\end{array}\right.
$$

seja positivo, então teremos que o primeiro autovalor, $\mu_{1}(k)$, associado ao operador $B$ satisfará

$$
\mu_{1}(k)=\mu_{1}>0
$$

O próximo resultado é um lema técnico, e sua prova pode ser encontrada em $[3,17]$.

Lema 2.4 Consideremos o operador $B$ definido em (2.13) com $c$ dado na Observação 2.3, tal que seu primeiro autovalor, $\mu_{1}(k)=\mu_{1}$, seja positivo. Sejam $\varepsilon, \alpha_{0}$ constantes positivas e $q \in(1, \infty)$. Então existe uma constante $M_{1}=M_{1}\left(B, \mu_{1}, \varepsilon, N, q, \alpha_{0}\right)$ tal que para todo $\alpha_{0} \geq \alpha \geq \beta \geq-\alpha_{0}$ temos

$$
\left\|e^{-B t} u_{0}\right\|_{X_{q}^{\alpha}} \leq M_{1} e^{-\left(\mu_{1}-\epsilon\right) t} t^{-(\alpha-\beta)}\left\|u_{0}\right\|_{X_{q}^{\beta}}, \quad t>0, \quad u_{0} \in X_{q}^{\beta},
$$

onde $\left\{X_{q}^{\alpha} ; \alpha \in \mathbb{R}, 1<q<\infty\right\}$ é a escala de espaços de Banach associadas ao operador B. Em particular, existe $\delta_{0}=\delta_{0}(q, N)>0$ e uma constante $\tilde{M}_{1}=\tilde{M}_{1}\left(B, \mu_{1}, \varepsilon, N, q\right)$ tal que

$$
\left\|e^{-B t} u_{0}\right\|_{L^{\infty}\left(\Omega, \mathbb{R}^{2}\right)} \leq \bar{M}_{1} e^{-\left(\mu_{1}-\varepsilon\right) t} t^{-\delta_{0}}\left\|u_{0}\right\|_{L^{q}\left(\Omega, \mathbb{R}^{2}\right)}, \quad t>0, \quad u_{0} \in L^{q}\left(\Omega, \mathbb{R}^{2}\right) .
$$

Ainda, se $q=2$, então as constantes $M_{1}$ e $\tilde{M}_{1}$ não dependem de ninguém.

Teorema 2.5 Suponhamos que as funções $f_{i}, g_{i} i=1,2$ satisfaçam (C1) ou (C2), respectivamente. Temos

(i) Se para $i=1,2 f_{i}$ e $g_{i}$ satisfazem (2.10) então, as soluções locais encontradas nos Teoremas 1.10 e 1.11, para o problema (2.9), são globalmente definidas.

(ii) Suponhamos ainda que o primeiro autovalor, $\lambda_{1}$, de (2.11) seja positivo. Então, existem constantes positivas $K_{\infty}$ e $K_{r}^{\alpha}$, com $\alpha<\frac{1}{2}+\frac{1}{2 r}$ e $r \geq q$, tais que

$$
\limsup _{t \rightarrow \infty}\left\|u\left(t ; u_{0}\right)\right\|_{L^{\infty}\left(\Omega, \mathbb{R}^{2}\right)} \leq K_{\infty}
$$




$$
\limsup _{t \rightarrow \infty}\left\|u\left(t ; u_{0}\right)\right\|_{X_{r}^{\alpha}} \leq K_{r}^{\alpha},
$$

com o limite uniforme para $u_{0}$ em subconjuntos limitados de $L^{q}\left(\Omega, \mathbb{R}^{2}\right)$ ou $W^{1, q}\left(\Omega, \mathbb{R}^{2}\right)$, respectivamente.

(iii) Se as hipóteses de (i) e (ii) estão satisfeitas e se $\phi=\left(\phi_{1}, \phi_{2}\right)$ é a solução do seguinte problema elíptico

$$
\begin{aligned}
& -\triangle \phi_{1}+C_{0} \phi_{1}=C_{1} \quad \text { em } \Omega, \\
& -\triangle \phi_{2}+C_{0} \phi_{2}=C_{1} \quad \text { em } \Omega, \\
& \frac{\partial \phi_{1}}{\partial \vec{n}}+B_{0} \phi_{1}+k\left(\phi_{1}-\phi_{2}\right)=B_{1} \quad \text { em } \Gamma, \\
& \frac{\partial \phi_{2}}{\partial \vec{n}}+B_{0} \phi_{2}-k\left(\phi_{1}-\phi_{2}\right)=B_{1} \quad \text { em } \Gamma .
\end{aligned}
$$

Então, $0 \leq \phi_{1}, \phi_{2} \in L^{\infty}(\Omega)$ e se $u\left(t, x ; u_{0}\right)=\left(u_{1}\left(t, x ; u_{0}\right), u_{2}\left(t, x ; u_{0}\right)\right)$ é a solução de (2.1) com dado inicial $u_{0}=\left(u_{0}^{1}, u_{0}^{2}\right)$, temos para $i=1,2$

$$
\lim _{t \rightarrow \infty}\left|u_{i}\left(t, x ; u_{0}\right)\right| \leq \phi_{i}(x),
$$

uniformente em $x \in \bar{\Omega}$ e para $u_{0}$ em subconjuntos limitados de $L^{q}\left(\Omega, \mathbb{R}^{2}\right)$ ou $W^{1, q}\left(\Omega, \mathbb{R}^{2}\right)$. De fato, se para $i=1,2,\left|u_{0}^{i}\right| \leq \phi_{i}(x)$ para todo $x \in \bar{\Omega}$ então, para $i=1,2$,

$$
\left|u_{i}\left(t, x, u_{0}\right)\right| \leq \phi_{i}(x), \quad \forall x \in \bar{\Omega}, \quad \forall t \geq 0
$$

Demonstração: Seja $\mathcal{B}$ um subconjunto limitado de $L^{q}\left(\Omega, \mathbb{R}^{2}\right)$, ou de $W^{1, q}\left(\Omega, \mathbb{R}^{2}\right)$, e suponhamos que $u_{0} \in \mathcal{B}$. Para mostrarmos (i), sejam $w_{0}=\left(\left|u_{0}\right|,\left|v_{0}\right|\right)$ e $w^{+}\left(t, w_{0}\right)=$ 
$\left(w_{1}^{+}\left(t ; w_{0}\right), w_{2}^{+}\left(t ; w_{0}\right)\right)$, a solução de

$$
\begin{array}{ll}
\left(w_{1}\right)_{t}-\Delta w_{1}=-C_{0} w_{1}+C_{1} & \text { em } \Omega, \\
\left(w_{2}\right)_{t}-\Delta w_{2}=-C_{0} w_{2}+C_{1} & \text { em } \Omega, \\
\frac{\partial w_{1}}{\partial \vec{n}}+k\left(w_{1}-w_{2}\right)=-B_{0} w_{1}+B_{1} & \text { em } \Gamma, \\
\frac{\partial w_{2}}{\partial \vec{n}}-k\left(w_{1}-w_{2}\right)=-B_{0} w_{2}+B_{1} & \text { em } \Gamma, \\
w\left(0 ; w_{0}\right)=w_{0} & \text { em } \Omega .
\end{array}
$$

Como $0 \leq C_{1}, B_{1}$, a Proposição 2.3, item (i) implica que $0 \leq w_{1}^{+}\left(t ; w_{0}\right), w_{2}^{+}\left(t ; w_{0}\right)$. Ainda, (2.10) implica que para $i=1,2 f_{i}(s) \leq-C_{0} s+C_{1}$ e $g_{i}(s) \leq-B_{0} s+B_{1}$. Então da Proposição 2.3, item (iii) obtemos que

$$
\begin{aligned}
& u_{1}\left(t ; u_{0}\right) \leq w_{1}^{+}\left(t ; w_{0}\right) \\
& u_{2}\left(t ; u_{0}\right) \leq w_{2}^{+}\left(t ; w_{0}\right)
\end{aligned}
$$

enquanto $u\left(t ; u_{0}\right)=\left(u_{1}\left(t ; u_{0}\right), u_{2}\left(t ; u_{0}\right)\right)$ existir. Também, com um raciocínio semelhante, obtemos que

$$
\begin{aligned}
& u_{1}\left(t ; u_{0}\right) \geq w_{1}^{-}\left(t ;-w_{0}\right)=-w_{1}^{+}\left(t ; w_{0}\right) \\
& u_{2}\left(t ; u_{0}\right) \geq w_{2}^{-}\left(t ;-w_{0}\right)=-w_{2}^{+}\left(t ; w_{0}\right)
\end{aligned}
$$

Portanto,

$$
\begin{aligned}
& \left|u_{1}\left(t ; u_{0}\right)\right| \leq w_{1}^{+}\left(t ; w_{0}\right) \\
& \left|u_{2}\left(t ; u_{0}\right)\right| \leq w_{2}^{+}\left(t ; w_{0}\right)
\end{aligned}
$$

enquanto $u\left(t ; u_{0}\right)=\left(u_{1}\left(t ; u_{0}\right), u_{2}\left(t ; u_{0}\right)\right)$ existir.

Seja $\phi=\left(\phi_{1}, \phi_{2}\right)$ a solução de (2.16), e defina $v=w^{+}-\phi$. Então $v$ satisfaz o problema linear homogêneo associado à (2.17). Logo, o Lema 2.4 implica que

$$
\|v(t)\|_{L^{\infty}\left(\Omega, \mathbf{R}^{2}\right)} \leq M_{1} t^{-\delta_{0}}\left\|w_{0}-\phi\right\|_{L q\left(\Omega, \mathbf{R}^{2}\right)} .
$$


Como

$$
\left\|w^{+}\left(t ; w_{0}\right)\right\|_{L^{\infty}\left(\Omega, \mathbb{R}^{2}\right)} \leq\|v(t)\|_{L^{\infty}\left(\Omega, \mathbb{R}^{2}\right)}+\|\phi\|_{L^{\infty}\left(\Omega, \mathbb{R}^{2}\right)}
$$

a norma $L^{\infty}\left(\Omega, \mathbb{R}^{2}\right)$ de $u\left(t ; u_{0}\right)$, fica limitada em intervalos limitados de tempo contidos em $(0, \infty)$.

Usando a fórmula da variação das constantes para a solução $u\left(t ; u_{0}\right)$, dada na Definição 1.1 temos, para $t>t_{0}>0$ arbitrários, que

$$
u\left(t ; u_{0}\right)=e^{-B\left(t-t_{0}\right)} u\left(t_{0} ; u_{0}\right)+\int_{t_{0}}^{t} e^{-B(t-s)} H\left(u\left(s ; u_{0}\right)\right) d s
$$

onde $B$ é o operador definido em (2.13) e

$$
\langle H(u), \psi)=\int_{\Omega} F(u(x)) \psi(x)+\int_{\Gamma} G(u(x)) \psi(x),
$$

com $F, G$ são definidas por

$$
\begin{aligned}
& F(v)=\left(c v_{1}+f_{1}\left(v_{1}\right), c v_{2}+f_{2}\left(v_{2}\right)\right) \\
& G(v)=\left(g_{1}\left(v_{1}\right)-k\left(v_{1}-v_{2}\right), g_{2}\left(v_{2}\right)+k\left(v_{1}-v_{2}\right)\right)
\end{aligned}
$$

para $v=\left(v_{1}, v_{2}\right) \in \mathbb{R}^{2}, c>0$ como na Observação 2.3 e $\psi$ adequado. Observemos que se $u \in L^{\infty}\left(\Omega, \mathbb{R}^{2}\right)$ e $\psi \in X_{q^{\prime}}^{r}$ com $2 r>\frac{1}{q^{\prime}}$ então, $\psi$ tem traço definido em $\Gamma$ e existe uma constante positiva $C:=C\left(\|u\|_{L^{\infty}\left(\Omega, \mathbb{R}^{2}\right)}\right)$ tal que

$$
\|H(u)\|_{X_{q}^{-r}} \leq C\left(\|u\|_{L^{\infty}\left(\Omega, \mathbb{R}^{2}\right)}\right)<\infty
$$

Portanto,

$$
\left\|u\left(t ; u_{0}\right)\right\|_{X_{q}^{\circ}} \leq M\left(t-t_{0}\right)^{-\alpha}\left\|u\left(t ; u_{0}\right)\right\|_{L^{q}\left(\Omega, \mathbb{R}^{2}\right)}+M C \int_{t_{0}}^{t}(t-s)^{-(\alpha+r)} d s .
$$

Logo, se $\alpha+r<1$, a norma em $X_{q}^{\alpha}$ de $u\left(t ; u_{0}\right)$ fica limitada em intervalos limitados de tempo. Portanto, para $\alpha<\frac{1}{2}+\frac{1}{2 q}$, segue da Proposiçāo 1.4 , que a solução $u\left(t ; u_{0}\right)$ é globalmente definida.

Para mostramos (ii), observemos que quando o primeiro autovalor, $\lambda_{1}$, de (2.11) é positivo. Segue do Lema 2.3, que o operador elíptico em (2.16) é positivo e, como $B_{1}, C_{1} \geq 0$ obtemos que $\phi_{1}, \phi_{2} \geq 0$. Ainda, o Lema 2.4 com $\varepsilon=\frac{\mu_{1}}{2}$ implica que

$$
\|v(t)\|_{L^{\infty}\left(\Omega, \mathbb{R}^{2}\right)} \leq M_{1} e^{-t \frac{\mu_{1}}{2}} t^{-\delta_{0}}\left\|w_{0}-\phi\right\|_{L^{q}\left(\Omega, \mathbb{R}^{2}\right)},
$$


daí

$$
\left\|w^{+}\left(t ; w_{0}\right)\right\|_{L^{\infty}\left(\Omega, \mathbb{R}^{2}\right)} \leq\|\dot{v}(t)\|_{L^{\infty}\left(\Omega, \mathbb{R}^{2}\right)}+\|\phi\|_{L^{\infty}\left(\Omega, \mathbb{R}^{2}\right)} \rightarrow\|\phi\|_{L^{\infty}\left(\Omega, \mathbb{R}^{2}\right)},
$$

quando $t \rightarrow \infty$, uniformemente para $u_{0} \in \mathcal{B}$, donde obtemos (2.14).

Para mostrarmos (2.15), observemos primeiro que se $t \geq t_{0}>0$, onde $t_{0}$ é tomado suficientemente grande tal que

$$
\left\|u\left(t ; u_{0}\right)\right\|_{L^{\infty}\left(\Omega, \mathbb{R}^{2}\right)} \leq K_{\infty}+\eta, \quad s \geq t_{0},
$$

então para $\alpha<1 / 2+1 / 2 r$ e $p>1 / 2 q^{\prime}$, com o mesmo raciocínio utilizado acima, segue que

$$
\begin{aligned}
\left\|u\left(s ; u_{0}\right)\right\|_{X_{q}^{\alpha}} \leq & M e^{-\mu_{1}\left(t-t_{0}\right)}\left(t-t_{0}\right)^{\alpha}\left\|u\left(t_{0} ; u_{0}\right)\right\|_{L^{\infty}\left(\Omega, \mathbb{R}^{2}\right)} \\
& +M C\left(K_{\infty}\right) \int_{t_{0}}^{t} e^{-\mu_{1}(t-s)}(t-s)^{-(\alpha+p)} d s .
\end{aligned}
$$

Assim,

$$
\left\|u\left(t ; u_{0}\right)\right\|_{X_{q}^{\alpha}} \leq M_{0} C\left(K_{\infty}\right) \int_{0}^{\infty} e^{-\frac{\mu}{2} \theta} \theta^{-(\alpha+p)} d \theta
$$

Agora,

$$
X_{q}^{\alpha} \hookrightarrow L^{s}\left(\Omega, \mathbb{R}^{2}\right)
$$

para algum $s:=s(q, \alpha)>q, F$ e $G$ são subcríticas em $L^{s}\left(\Omega, \mathbb{R}^{2}\right)$, então com o mesmo raciocínio acima, obtemos que para todo $r \in[q, s]$ e $\alpha<1 / 2+1 / 2 r$, existe uma constante positiva $K_{\alpha}^{r}$ tal que (2.15) é válida. Um argumento de "bootstrap" mostra que (2.15) continua válida para $r \geq q$ e $\alpha<1 / 2+1 / 2 r$.

Finalmente, usando (2.18) e o fato de $v(t)$ convergir para zero, uniformemente para $u_{0} \in \mathcal{B}$, obtemos que

$$
\limsup _{t \rightarrow \infty}\left|u_{i}\left(t, x ; u_{0}\right)\right| \leq \phi_{i}, \quad \forall x \in \Omega,
$$

uniformemente para $u_{0} \in B$. Por outro lado, se $\left|u_{0}^{i}\right| \leq \phi_{i}, i=1,2$ então, $v_{i}(0) \leq 0$ e, portanto para $i=1,2$

$$
\left|u_{i}\left(t, x ; u_{0}\right)\right| \leq \phi_{i}, \forall x \in \bar{\Omega} \text { e } \forall t \geq 0,
$$

concluíndo a prova de (iii).

Do Teorema 2.5 e dos resultados de [13], temos o seguinte resultado. 
Teorema 2.6 Seja $X=L^{q}\left(\Omega, \mathbb{R}^{2}\right)$ ou $X=W^{1, q}\left(\Omega, \mathbb{R}^{2}\right)$, e suponhamos que para $i=1,2, f_{i}$ e $g_{i}$ satisfaçam $(\mathrm{C} 1)$ e (C2), e (2.10). Então, o problema (2.1) tem um atrator compacto global $\mathcal{A}_{X}$ tal que

$$
\mathcal{A}_{X} \subset \Sigma\left(\phi_{1}, \phi_{2}\right):=\left\{u \in L^{\infty}\left(\Omega, \mathbb{R}^{2}\right) ;\left|u_{i}(x)\right| \leq \phi_{i}(x), \forall x \in \Omega, \quad i=1,2\right\} .
$$

Ainda mais, para $2 \alpha<1+\frac{1}{r}$ e $r \geq q, \mathcal{A}_{X} \subset X_{r}^{\alpha}$, é compacto neste espaço e atrai subconjuntos limitados de $X$, na norma de $X_{r}^{\alpha}$.

Demonstração: Seja $\mathcal{B}$ um subconjunto limitado de $X$. Denotemos por $T(t) u_{0}:=$ $u\left(t ; u_{0}\right)$, o semigrupo gerado por (2.9). O item (ii) do Teorema 2.5 implica que $T(t) \mathcal{B}$ é um subconjunto limitado de $L^{\infty}\left(\Omega, \mathbb{R}^{2}\right)$. Também, o item (iii) do Teorema 2.5 implica que

$$
T(t) \mathcal{B} \subset \Sigma\left(\phi_{1}, \phi_{2}\right)
$$

para $t$ suficientemente grande, onde $\Sigma\left(\phi_{1}, \phi_{2}\right)$ definido acima. Logo o Teorema 3.4.6 de [13], demonstra a primeira parte do teorema.

Lembrando que $X_{r}^{\alpha}$, para $\alpha$ e $r$ nas condições do teorema, está compactamente imerso em $X$, e que o Teorema 2.4 mostra a existência de um subconjunto précompacto absorvente em $X_{r}^{\alpha}$, os argumentos utilizados na primeira parte mostram o teorema.

Nosso objetivo agora é encontra estimativas, independentes da constante $k$, para pontos do atrator global $\mathcal{A}_{X}$, dado no Teorema 2.6. Precisamos antes do seguinte lema.

Lema 2.5 Seja $\phi=\left(\phi_{1}, \phi_{2}\right) \in H^{1}\left(\Omega, \mathbb{R}^{2}\right)$ uma solução fraca do problema elíptico (2.16). Suponhamos que o primeiro autovalor, $\lambda_{1}$, de (2.11) seja positivo. Então, $\phi \in L^{\infty}\left(\Omega, \mathbb{R}^{2}\right)$ e para $i=1,2$

$$
\left\|\phi_{i}\right\|_{L^{\infty}(\Omega)} \leq C\left(\left|B_{1}\right|+\left|C_{1}\right|\right),
$$

onde $C=C\left(|\Omega|, \lambda_{1}, N\right)$. Ainda, existe $k_{0}>0$ suficientemente grande tal que a constante $C$ em (2.19) pode ser escolhida independente de $k$, para todo $k>k_{0}$.

Demonstração: Como $\lambda_{1}>0$, o Lema 2.3 implica que $\lambda_{1}(k)=\lambda_{1}>0$ é positivo, para todo $k>0$. Introduzindo a mudança de variáveis $\psi_{1}=\phi_{1}-\phi_{2}$ e $\psi_{2}=\phi_{1}+\phi_{2}$ 
obtemos que o problema (2.16) pode ser escrito na forma

$$
\begin{aligned}
& -\Delta \psi_{1}+C_{0} \psi_{1}=0 \quad \text { em } \quad \Omega, \\
& -\triangle \psi_{2}+C_{0} \psi_{2}=2 C_{1} \quad \text { em } \quad \Omega, \\
& \frac{\partial \psi_{1}}{\partial \vec{n}}+B_{0} \psi_{1}+2 k \psi_{1}=0 \quad \text { em } \quad \Gamma, \\
& \frac{\partial \psi_{2}}{\partial \vec{n}}+B_{0} \psi_{2}=2 B_{1} \quad \text { em } \quad \Gamma .
\end{aligned}
$$

Como o problema acima é desacoplado, O Lema 8.1, item (iii) de [6] implica que

$$
\left\|\psi_{2}\right\|_{L^{\infty}(\Omega)} \leq C_{2}\left(\left|B_{1}\right|+\left|C_{1}\right|\right)
$$

onde $C_{2}=C_{2}\left(|\Omega|, \lambda_{1}, N\right)$. O resultado segue então do fato de $\psi_{1} \equiv 0$.

Mostraremos no Capítulo 3 uma adaptação do Lema 8.1 de [6], veja Proposição 3.1. Finalmente, do Teorema 2.6 e do Lema 2.5 temos o

Teorema 2.7 Seja $X=L^{q}\left(\Omega, \mathbb{R}^{2}\right)$ ou $X=W^{1, q}\left(\Omega, \mathbb{R}^{2}\right)$. Para $i=1,2$ suponhamos que as funções $f_{i}, g_{i}$ satisfaçam as condições (C1), (C2), e (2.10). Suponhamos ainda, que o primeiro autovalor, $\lambda_{1}$, de (2.11) seja positivo. Então,

$$
\sup _{v \in \mathcal{A}_{X}}\|v\|_{L^{\infty}\left(\Omega, \mathbb{R}^{2}\right)} \leq K_{0}
$$

onde $K_{0}=K_{0}\left(|\Gamma|, \lambda_{1}, C_{0}, C_{1}, B_{0}, B_{1}\right)$

Consideremos, agora, o problema

$$
\left\{\begin{array}{l}
u_{t}(t, x)=D \Delta u(t, x)+F(u(t, x)) \quad t>0, \quad x \in \Omega, \\
D \frac{\partial u}{\partial \vec{n}}(t, x)=G(u(t, x)) \quad t>0, \quad x \in \Gamma,
\end{array}\right.
$$

onde $u, k, \Omega, \Gamma$ são como definidos para (1.1), $D$ é a seguinte matriz $n \times n$ :

$$
D=\left[\begin{array}{lllll}
d_{1} & 0 & 0 & \cdots & 0 \\
0 & d_{2} & 0 & \cdots & 0 \\
0 & 0 & d_{3} & \cdots & 0 \\
\vdots & \vdots & \vdots & \ddots & \vdots \\
0 & 0 & 0 & \cdots & d_{n}
\end{array}\right]
$$


com $d_{i}>0$, para todo $i=1,2, \cdots, n$ e $F=\left(F_{1}, F_{2}, \cdots, F_{n}\right): \operatorname{IR}^{n} \rightarrow \operatorname{IR}^{n}$ e $G=$ $\left(G_{1}, G_{2}, \cdots, G_{n}\right): \operatorname{IR}^{n} \rightarrow \operatorname{IR}^{n}$ são duas funções como definidas em (2.8), tais que para todo $u=\left(u_{1}, \cdots, u_{n}\right) \in \mathbb{I R}^{n}$

$$
\begin{aligned}
& u_{i} f_{i}(u) \leq-C_{0} u_{i}^{2}+C_{1}\left|u_{i}\right| \\
& u_{i} g_{i}(u) \leq-B_{0} u_{i}^{2}+B_{1}\left|u_{i}\right|
\end{aligned}
$$

$i=1,2, \cdots, n$. Tomando a matriz de acoplamento $K_{n} \equiv 0$, na definição do operador $\tilde{B}$ em (2.3), a Proposição 2.3 garante resultados de comparação e positividade para o soluçōes do problema (2.20), e com os mesmos argumentos utilizados nesta seção obtemos o seguinte teorema:

Teorema 2.8 Suponhamos que para $i=1,2, \cdots, n f_{i}, g_{i}$ satisfaçam as condições de crescimento (C1), ou (C2) e (2.21). Então,

(i) Para cada $u_{0} \in L^{q}\left(\Omega, \mathbb{R}^{n}\right)$ ou $u_{0} \in W^{1, q}\left(\Omega, \mathbb{R}^{n}\right)$ respectivamente, as soluções encontradas para o problema (2.20), são globalmente definidas.

(ii) Suponhamos ainda que o primeiro autovalor, $\lambda_{1}(D)$, do problema

$$
\begin{array}{ll}
-D \Delta u+C_{0} u=\lambda u & \text { em } \\
D \frac{\partial u}{\partial \vec{n}}+B_{0} u=0 & \text { em } \Gamma,
\end{array}
$$

seja positivo, onde $D$ é como acima, $C_{0}$ e $B_{0}$ são dados em (2.21). Então o problema (2.20) possui um atrator global compacto $\mathcal{A}_{X}(D)$ tal que

$$
\mathcal{A}_{X}(D) \subset \Sigma(\phi):=\left\{\phi \in L^{\infty}\left(\Omega, \mathbb{R}^{n}\right):\left|u_{i}(x)\right| \leq \phi_{i}(x), i=1,2, \cdots, n x \in \Omega\right\}
$$

onde $\phi$ é a solução do problema elíptico:

$$
\begin{aligned}
& -D \Delta \phi+C_{0} \phi=C_{1} \quad \text { em } \quad \Omega, \\
& D \frac{\partial \phi}{\partial \vec{n}}+B_{0} \phi=B_{1} \quad \text { em } \Gamma,
\end{aligned}
$$

onde $C_{0}, C_{1}, B_{0}$ e $B_{1}$ são dadas em (2.21).

Ainda mais, para $2 \alpha<1+\frac{1}{r}$, er $\geq q, \mathcal{A}_{X}(D) \subset E_{r}^{\alpha}$, é compacto neste espaço e atrai subconjuntos limitados de $X$, na norma de $E_{r}^{\alpha}$. 


\subsection{Semicontinuidade Superior de Atratores}

Como consequência imediata dos resultados da Seção 2.2, podemos mostrar uma propriedade simples das soluções do seguinte problema: seja $\Omega \subset \mathbb{R}^{N}, N \geq 1$, um domínio limitado com fronteira $\Gamma:=\partial \Omega$ suave, seja

$$
\left\{\begin{array}{l}
u_{t}(t, x)=D \Delta u(t, x)+F(u(t, x)) \quad t>0, \quad x \in \Omega \\
D \frac{\partial u}{\partial \bar{n}}(t, x)=\lambda G(u(t, x)) \quad t>0, \quad x \in \Gamma
\end{array}\right.
$$

onde

- $\vec{n}$ é a normal à $\Gamma$, apontando para fora de $\Omega$;

- $D$ é a matriz $n \times n$ definida em (2.6) tal que $d_{i}=1, i=1,2, \cdots, n$;

- $F: \mathbb{R}^{n} \rightarrow \mathbb{R}^{n}$ e $G: \mathbb{R}^{n} \rightarrow \mathbb{R}^{n}$ säo as funções definidas em (2.8);

- $\lambda$ é um parâmetro não negativo.

Os resultados dos Capítulo 1 e das seções anteriores implicam que o problema (2.23) possui uma única solução globalmente definida, dada pela fórmula da variação das constantes

$$
T_{\lambda}(t) u_{0}=e^{-A t} u_{0}+\int_{0}^{t} e^{-A(t-s)} h_{\lambda}\left(T_{\lambda}(s) u_{0}\right) d s,
$$

para $u_{0} \in X$, onde $X=L^{q}\left(\Omega, \mathbb{R}^{n}\right)$ ou $X=W^{1, q}\left(\Omega, \mathbb{R}^{n}\right), 1<q<\infty$, e o operador $A: D(A) \subset L^{q}\left(\Omega, \mathbb{R}^{n}\right) \rightarrow L^{q}\left(\Omega, \mathbb{R}^{n}\right)$, é definido por

$$
\begin{aligned}
& D(A)=\left\{\phi \in W^{2, q}\left(\Omega, \mathbb{R}^{n}\right) ; \frac{\partial \phi}{\partial \bar{n}}=0\right\} \\
& A \phi=-\triangle \phi+c \phi
\end{aligned}
$$

lembrando que $c>0$, é tomado de tal forma que o primeiro autovalor associado ao operador $A$ seja positivo, e a função $h_{\lambda}:=F_{\Omega}+G_{\Gamma}$, atuando em funções testes, $\phi$, adequadas da seguinte forma:

$$
\left\langle h_{\lambda}(u), \phi\right\rangle:=\left\langle f_{\Omega}(u), \phi\right\rangle+\left\langle g_{\Gamma}(u), \phi\right\rangle=\int_{\Omega}(F(u(x))+c u(x)) \phi(x)+\int_{\Gamma} \lambda G(u(x)) \phi(x)
$$


Ainda mais, para todo $\lambda \geq 0$, o problema (2.23) possui um atrator global compacto, denotado por $\mathcal{A}_{X}(\lambda)$, em $X$ tal que para $2 \alpha<1+\frac{1}{r}$, e $r \geq q, \mathcal{A}_{X}(\lambda) \subset E_{r}^{\alpha}$, é compacto neste espaço e atrai conjuntos limitados de $X$, na norma de $E_{r}^{\alpha}$, onde $E_{q}^{\alpha}, \alpha \in[-1,1] \mathrm{e}$ $1<q<\infty$, é a escala de espaços de Banach associadas ao operador $A$, definido acima, como construída na Seção 1.1.7. Também,

$$
\left\|e^{-A t} u_{0}\right\|_{E_{r}^{\alpha}} \leq M e^{-\frac{\mu_{1}}{2} t} t^{-(\alpha-\beta)}\left\|u_{0}\right\|_{E_{r}^{\beta}}, \quad t>0, u_{0} \in E_{r}^{\beta},
$$

onde $\mu_{1}$ é o primeiro autovalor associado ao operador $A, \alpha \geq \beta$ e $r$ satisfazendo as restriçoes acima e $M=M\left(N, \mu_{1}, \alpha, \beta, r\right)>0$.

Vamos mostar que a família de atratores globais $\left\{\mathcal{A}_{X}(\lambda), \quad 0 \leq \lambda<\infty\right\}$ é semicontínua superiormente em $\lambda=0$, conforme a próxima definiçāo, veja [13].

Definição 2.5 Diremos que a familia de atratores globais $\left\{\mathcal{A}_{X}(\lambda), \quad 0 \leq \lambda<\infty\right\}$ é semicontínua superiormente em $\lambda=0$, se para toda $\varepsilon>0$, existir $\lambda_{0}>0$ tal que

$$
\sup _{u \in \mathcal{A}_{X}(\lambda)} d_{E_{q}^{\alpha}}\left(u, \mathcal{A}_{X}(0)\right) \leq \varepsilon
$$

para todo $\lambda \leq \lambda_{0}$, onde $d_{E_{q}^{\alpha}}(\cdot, \cdot)$ é a distância em $E_{q}^{\alpha}$.

Observemos primeiro que o item (ii) do Teorema 2.8, juntamente com o Lema 2.5 com $k=0$ e $n \geq 1$, implicam que existe $\lambda_{0}>0$ e um conjunto limitado, $\Sigma_{0} \subset \mathbb{R}^{n}$, tais que

$$
\mathcal{A}_{X}(\lambda) \subset \Sigma_{0}
$$

para todo $0 \leq \lambda \leq \lambda_{0}$, onde $\Sigma_{0}=\Sigma_{0}\left(C_{0}, C_{1}, B_{0}, B_{1}, \lambda_{0}\right)$. Dessa forma, podemos assumir que existam constantes positivas $L_{f}$ e $L_{g}$, independentes de $\lambda$, para $0 \leq \lambda \leq \lambda_{0}$, tais que

$$
|F(u)-F(v)| \leq L_{f}|u-v| \quad \text { e } \quad|G(u)-G(v)| \leq L_{g}|u-v|
$$

para todo $u, v \in \mathbb{R}^{n}$.

Sejam $\alpha$ e $r$ tais que

$$
2 \alpha<1+\frac{1}{r} \quad \text { e } \quad r \geq q .
$$


Assim, se $\psi_{\lambda} \in \mathcal{A}_{X}(\lambda) \subset E_{\alpha}^{r}$, então

$$
\begin{aligned}
T_{\lambda}(t) \psi_{\lambda}-T_{0}(t) \psi_{\lambda}= & \int_{0}^{t} e^{-A(t-s)} h_{\lambda}\left(T_{\lambda}(s) \psi_{\lambda}\right) d s-\int_{0}^{t} e^{-A(t-s)} h_{0}\left(T_{0}(s) \psi_{\lambda}\right) d s \\
= & \int_{0}^{t} e^{-A(t-s)}\left[h_{\lambda}\left(T_{\lambda}(s) \psi_{\lambda}\right)-h_{0}\left(T_{\lambda}(s) \psi_{\lambda}\right)\right] d s \\
& +\int_{0}^{t} e^{-A(t-s)}\left[h_{0}\left(T_{\lambda}(s) \psi_{\lambda}\right)-h_{0}\left(T_{0}(s) \psi_{\lambda}\right)\right] d s \\
= & \lambda \int_{0}^{t} e^{-A(t-s)}\left[G\left(T_{\lambda}(s) \psi_{\lambda}\right)+h_{0}\left(T_{\lambda}(s) \psi_{\lambda}\right)-h_{0}\left(T_{0}(s) \psi_{\lambda}\right)\right] d s
\end{aligned}
$$

Logo, usando (2.24) obtemos que

$$
\begin{aligned}
\left\|T_{\lambda}(t) \psi_{\lambda}-T_{0}(t) \psi_{\lambda}\right\|_{E_{r}^{\alpha}} \leq & \lambda\left\|\int_{0}^{t} e^{-A(t-s)} g\left(T_{\lambda}(s) \psi_{\lambda}\right) d s\right\|_{E_{r}^{\alpha}} \\
& +\left\|\int_{0}^{t} e^{-A(t-s)}\left[h_{0}\left(T_{\lambda}(s) \psi_{\lambda}\right)-h_{0}\left(T_{0}(s) \psi_{\lambda}\right)\right] d s\right\|_{E_{r}^{\alpha}} \\
\leq & \lambda M \int_{0}^{t} e^{-\frac{\mu_{1}}{2}(t-s)}\left\|G\left(T_{\lambda}(s) \psi_{\lambda}\right)\right\|_{E_{r}^{\alpha}} d s \\
& +M \int_{0}^{t} e^{-\frac{\mu_{1}}{2}(t-s)}\left\|h_{0}\left(T_{\lambda}(s) \psi_{\lambda}\right)-h_{0}\left(T_{0}(s) \psi_{\lambda}\right)\right\|_{E_{r}^{\alpha}} d s \\
\leq & \lambda M \int_{0}^{t} e^{-\frac{\mu_{1}}{2}(t-s)}\left\|G\left(T_{\lambda}(s) \psi_{\lambda}\right)\right\|_{E_{r}^{\alpha}} \| d s \\
& +M \int_{0}^{t} e^{-\frac{\mu_{1}}{2}(t-s)}\left\|h_{0}\left(T_{\lambda}(s) \psi_{\lambda}\right)-h_{0}\left(T_{0}(s) \psi_{\lambda}\right)\right\|_{E_{r}^{\alpha}} d s
\end{aligned}
$$

Como $\psi_{\lambda} \in \mathcal{A}_{X}(\lambda)$, então $T_{\lambda}(s) \psi_{\lambda} \in \mathcal{A}_{X}(\lambda)$, para todo $0 \leq s \leq t$ e, portanto, existe uma constante $K_{\mathrm{I}}>0$, independente de $\lambda$, para $0 \leq \lambda \leq \lambda_{0}$, tal que

$$
\left\|G\left(T_{\lambda}(s) \psi_{\lambda}\right)\right\|_{E_{\tau}^{\alpha}} \leq K_{1}
$$

da mesma forma existe uma constante $K_{2}>0$, independente de $\lambda$, para $0 \leq \lambda \leq \lambda_{0}$, tal que

$$
\left\|h_{0}\left(T_{\lambda}(s) \psi_{\lambda}\right)-h_{0}\left(T_{0}(s) \psi_{\lambda}\right)\right\|_{E_{r}^{\alpha}} \leq K_{2}\left\|T_{\lambda}(s) \psi_{\lambda}-T_{0}(s) \psi_{\lambda}\right\|_{E_{r}^{\alpha}}
$$

Assim,

$$
\left\|T_{\lambda}(t) \psi_{\lambda}-T_{0}(t) \psi_{\lambda}\right\|_{E_{r}^{\alpha}} \leq \lambda M K_{1}+M K_{2} \int_{0}^{t}\left\|T_{\lambda}(s) \psi_{\lambda}-T_{0}(s) \psi_{\lambda}\right\|_{E_{r}^{\alpha}} d s
$$


Portanto, a Desigualdade de Gronwall, veja [17], implica que

$$
\left\|T_{\lambda}(t) \psi_{\lambda}-T_{0}(t) \psi_{\lambda}\right\|_{E_{r}^{a}} \leq M K_{1} \lambda e^{t}, \quad t \geq 0
$$

Para todo $\varepsilon>0$, como $\mathcal{A}_{X}(0)$ atrai limitados de $X$, através do fluxo $T_{0}(t)$, na norma de $E_{r}^{\alpha}$, existe $\lambda_{0}=\lambda_{0}(\varepsilon)>0$ tal que se $0 \leq \lambda \leq \lambda_{0}$, então

$$
\sup _{\psi_{\lambda} \in \mathcal{A}_{X}(\lambda)} \operatorname{dist}_{E_{r}^{\alpha}}\left(T_{0}\left(t_{0}\right) \psi_{\lambda}, \mathcal{A}_{X}(0)\right) \leq \frac{\varepsilon}{2}
$$

para algum $t_{0}>0$. Diminuindo $\lambda_{0}>0$, se necessário, obtemos que para todo $0 \leq \lambda \leq$ $\lambda_{0}$,

$$
M K_{1} \lambda e^{t_{0}} \leq \frac{\varepsilon}{2}
$$

Portanto,

$$
\begin{aligned}
d_{E_{r}^{\alpha}}\left(T_{\lambda}\left(t_{0}\right) \psi_{\lambda}, \mathcal{A}_{X}(0)\right) & \leq d_{E_{r}^{\alpha}}\left(T_{\lambda}\left(t_{0}\right) \psi_{\lambda}, T_{0}\left(t_{0}\right) \psi_{\lambda}\right)+d_{E_{r}^{\alpha}}\left(T_{0}\left(t_{0}\right) \psi_{\lambda}, \mathcal{A}_{X}(0)\right) \\
& <\frac{\varepsilon}{2}+\frac{\varepsilon}{2}=\varepsilon
\end{aligned}
$$

para $0 \leq \lambda \leq \lambda_{0}$. Mas,

$$
T_{\lambda}\left(t_{0}\right) \mathcal{A}_{X}(\lambda)=\mathcal{A}_{X}(\lambda)
$$

para todo $\lambda \geq 0$. Assim, para $0 \leq \lambda \leq \lambda_{0}$,

$$
d_{E_{r}^{\alpha}}\left(\psi_{\lambda}, \mathcal{A}_{X}(0)\right)<\varepsilon
$$

Assim,

$$
\sup _{\psi_{\lambda} \in \mathcal{A}_{X}(\lambda)} d_{E_{\tilde{\sigma}}^{\alpha}}\left(\psi_{\lambda}, \mathcal{A}_{X}(0)\right)<\varepsilon
$$

para $0 \leq \lambda \leq \lambda_{0}$, o que mostra a semicontinuidade superior da família de atratores globais $\left\{\mathcal{A}_{X}(\lambda), \lambda \geq 0\right\}$, em $\lambda=0$. 


\section{Capítulo 3}

\section{Sincronização Através da Fronteira em Problemas Parabólicos com Condição de Fronteira não Linear}

Consideremos o problema

$$
\left\{\begin{array}{l}
u_{t}(t, x)=\Delta u(t, x)+f_{1}(u(t, x)) \quad t>0, \quad x \in \Omega, \\
v_{t}(t, x)=\Delta v(t, x)+f_{2}(v(t, x)) \quad t>0, \quad x \in \Omega, \\
\frac{\partial u}{\partial \vec{n}}(t, x)=g_{1}(u(t, x))-k(u(t, x)-v(t, x)) \quad t>0, \quad x \in \Gamma, \\
\frac{\partial v}{\partial \vec{n}}(t, x)=g_{2}(v(t, x))+k(u(t, x)-v(t, x)) \quad t>0, \quad x \in \Gamma,
\end{array}\right.
$$

para funções $f_{i}, g_{i}: \mathbb{R} \rightarrow \mathbb{R}, i=1,2$, satisfazendo as condições exigidas nos Capítulos 1 e 2 e tais que existam constantes positivas $C_{f}$ e $C_{g}$ satisfazendo

$$
\sup _{x \in \mathbb{R}}\left|f_{1}(x)-f_{2}(x)\right| \leq C_{f} \quad \text { e } \sup _{x \in \mathbb{R}}\left|g_{1}(x)-g_{2}(x)\right| \leq C_{g} .
$$

Os resultados do Capítulo 2 mostram que esta condição sobre a diferença entre $f_{1}, f_{2}$ e $g_{1}, g_{2}$ pode ser assumida sem perda da generalidade.

O objetivo deste capítulo é mostrarmos que existe uma constante positiva, suficientemente grande, $k_{0}$ tal que para todo $k>k_{0}$, toda solução $(u(t, \cdot), v(t, \cdot))$ de (3.1), 
satisfaz

$$
\limsup _{t \rightarrow \infty}\|u(t, \cdot)-v(t, \cdot)\|_{L^{\infty}(\Omega)} \leq C\left(\left|C_{f}\right|+\left|C_{g}\right|\right),
$$

com $C_{f}, C_{g}$ dados acima, e $C$ uma constante positiva independente de $k$. Observemos que se $f_{1}=f_{2}$ e $g_{1}=g_{2}$, uma consequência imediata da propriedade acima é que

$$
\lim _{t \rightarrow \infty}\|u(t, \cdot)-v(t, \cdot)\|_{L^{\infty}(\Omega)}=0 .
$$

Tal propriedade é chamada de sincronização. Sincronização de problemas parabólicos acoplados foi estudada em [9], para problemas parabólicos com condição de fronteira do tipo Neumann, e com acoplamento atuando dentro da região $\Omega$. Neste capítulo obteremos sincronização de problemas parabólicos com condição de fronteira não linear e acoplamento atuando na fronteira da região $\Omega$.

\subsection{Sincronização em Problemas Parabólicos}

Seja $\left(u\left(t ; u_{0}\right), v\left(t ; v_{0}\right)\right)$ a solução de $(3.1)$, com dado inicial $\left(u_{0}, v_{0}\right) \in X, \operatorname{com} X=$ $L^{q}\left(\Omega, \mathbb{R}^{2}\right)$ ou $X=W^{1, q}\left(\Omega, \mathbb{R}^{2}\right)$, conforme os Capítulos anteriores.

Os resultados da Seção 2.2 , mostram que existe uma região limitada $\Sigma_{0} \subset \mathbb{R}^{2}$ tal que para todo $k>0$,

$$
u\left(t ; u_{0}\right)(x), v\left(t ; v_{0}\right)(x) \in \Sigma_{0}
$$

para todo $x \in \Omega$, todo $t>0$ e todo $\left(u_{0}, v_{0}\right) \in X$, onde $X=L^{q}\left(\Omega, \mathbb{R}^{2}\right)$ ou $X=$ $W^{1, q}\left(\Omega, \mathbb{R}^{2}\right)$. Considere então, $L_{f}$ e $L_{g}$ constantes positivas tais que, para todo $(u, v) \in$ $\Sigma_{0}$ e $i=1,2$,

$$
\begin{aligned}
& \left|f_{1}(u)-f_{2}(v)\right| \leq L_{f}|u-v|, \\
& \left|g_{1}(u)-g_{2}(v)\right| \leq L_{g}|u-v| .
\end{aligned}
$$

Podemos então supor, sem perda da generalidade, que (3.2) e (3.3) são válidas para todo $u, v \in \mathbb{R}$.

Considerando a seguinte mudança de variáveis, $w=u-v$ e $z=u+v$, em (3.1), 
obtemos que $\left(w\left(t ; w_{0}\right), z\left(t ; z_{0}\right)\right)$ é solução do problema

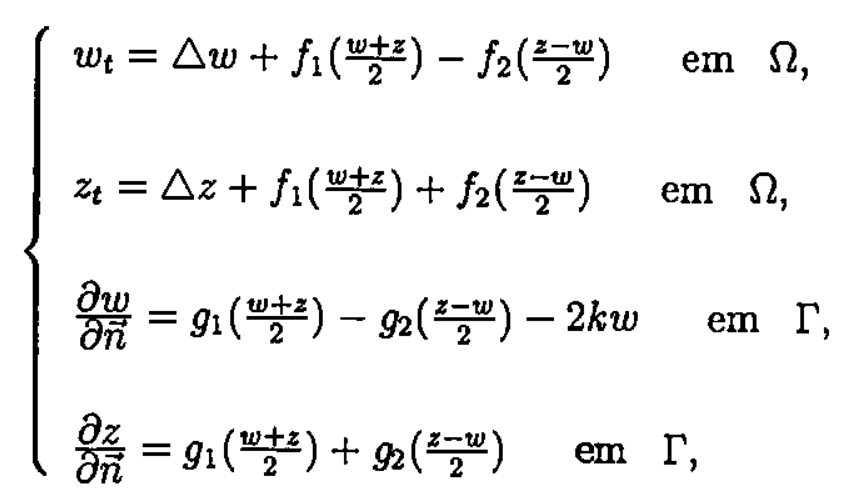

com dado inicial $\left(w_{0}, z_{0}\right)=\left(u_{0}-v_{0}, u_{0}+v_{0}\right) \in X$, O próximo resultado será essencial para o desenvolvimento desta seção.

Lema 3.1 Seja $\left(w\left(t ; w_{0}\right), z\left(t ; z_{0}\right)\right)$ uma solução de (3.4) com dado inicial $\left(w_{0}, z_{0}\right)$ definido acima. Então

$$
w\left(t, w_{0}\right)(x) \leq \bar{w}\left(t ;\left|w_{0}\right|\right)(x) \quad e \quad z\left(t, z_{0}\right)(x) \leq \bar{z}\left(t ;\left|z_{0}\right|\right)(x),
$$

para todo $t \geq 0$ e todo $x \in \Omega$, onde $\left(\bar{w}\left(t ;\left|w_{0}\right|\right), \bar{z}\left(t ;\left|z_{0}\right|\right)\right)$ é a solução do problema

$$
\left\{\begin{array}{lll}
\bar{w}_{t}=\Delta \bar{w}+L_{f}|\bar{w}|+C_{f} & \text { em } & \Omega, \\
\bar{z}_{t}=\Delta \bar{z}+f_{1}\left(\frac{\bar{w}+\bar{z}}{2}\right)+f_{2}\left(\frac{\bar{z}-\bar{w}}{2}\right) & \text { em } \Omega, \\
\frac{\partial \bar{w}}{\partial \bar{n}}=L_{g}|\bar{w}|+C_{g}-2 k \bar{w} & \text { em } & \Gamma, \\
\frac{\partial \bar{z}}{\partial \bar{n}}=g_{1}\left(\frac{\bar{w}+\bar{z}}{2}\right)+g_{2}\left(\frac{\bar{w}-\bar{z}}{2}\right) & \text { em } & \Gamma,
\end{array}\right.
$$

com dado inicial $\left(\bar{w}_{0}, \bar{z}_{0}\right)=\left(\left|w_{0}\right|,\left|z_{0}\right|\right)$.

Demonstraçāo: Para $z, w \in \mathbb{R}$, definamos

$$
\begin{aligned}
& F_{1}(z, w)=f_{1}\left(\frac{z+w}{2}\right)-f_{1}\left(\frac{z-w}{2}\right)+f_{1}\left(\frac{z-w}{2}\right)-f_{2}\left(\frac{z-w}{2}\right), \\
& F_{2}(z, w)=f_{1}\left(\frac{z+w}{2}\right)+f_{2}\left(\frac{z-w}{2}\right), \\
& G_{1}(z, w)=g_{1}\left(\frac{z+w}{2}\right)-g_{1}\left(\frac{z-w}{2}\right)+g_{1}\left(\frac{z-w}{2}\right)-g_{2}\left(\frac{z-w}{2}\right)-2 k w, \\
& G_{2}(z, w)=g_{1}\left(\frac{z+w}{2}\right)+g_{2}\left(\frac{z-w}{2}\right) .
\end{aligned}
$$


Então segue de (3.2) e (3.3), que para todo $z, w \in \mathbb{R}$,

$$
F_{1}(z, w) \leq L_{f}|w|+C_{f} \text { e } G_{1}(z, w) \leq L_{g}|w|+C_{g}-2 k w .
$$

Como $w_{0} \leq\left|w_{0}\right|$ e $z_{0} \leq\left|z_{0}\right|$, a Proposiçāo 2.3 item (iii), demonstra o lema.

Com modificações óbvias, mostramos o seguinte resultado.

Lema 3.2 Seja $\left(w\left(t ; w_{0}\right), z\left(t ; z_{0}\right)\right)$ uma solução de (3.4) com dado inicial $\left(w_{0}, z_{0}\right)$ definido acima. Então

$$
w\left(t, w_{0}\right)(x) \geq \underline{w}\left(t ;-\left|w_{0}\right|\right)(x) \quad e \quad z\left(t, z_{0}\right)(x) \geq \underline{z}\left(t ;-\left|z_{0}\right|\right)(x),
$$

para todo $t \geq 0$ e todo $x \in \Omega$, onde $\left(\underline{w}\left(t ;-\left|w_{0}\right|\right), \underline{z}\left(t ;-\left|z_{0}\right|\right)\right)$ é a solução do problema

$$
\left\{\begin{array}{l}
\underline{w}_{t}=\Delta \underline{w}-L_{f}|\underline{w}|-C_{f} \quad \text { em } \quad \Omega, \\
\underline{z}_{t}=\Delta \underline{z}+f_{1}\left(\frac{w+\underline{z}}{2}\right)+f_{2}\left(\frac{z-\underline{w}}{2}\right) \quad \text { em } \quad \Omega, \\
\frac{\partial \underline{w}=-L_{g}|\underline{w}|-C_{g}-2 k \underline{w}}{\partial \overline{\bar{n}}} \quad \text { em } \quad \Gamma \\
\frac{\partial z}{\partial \overline{\bar{n}}}=g_{1}\left(\frac{\underline{w}+\underline{z}}{2}\right)+g_{2}\left(\frac{\underline{w}-\underline{z}}{2}\right) \quad \text { em } \quad \Gamma,
\end{array}\right.
$$

com dado inicial $\left(\underline{w}_{0}, \underline{z}_{0}\right)=\left(-\left|w_{0}\right|,-\left|z_{0}\right|\right)$.

Estamos interessados em estimar a diferença $u\left(t ; u_{0}\right)(x)-v\left(t ; v_{0}\right)(x)$, para $t \geq 0$ suficientemente grande, e todo $x \in \Omega$. Assim, a partir de agora trabalharemos apenas com as desigualdades para $w\left(t ; w_{0}\right)$ dadas nos Lemas 3.1 e 3.2 .

Como $\left|w_{0}\right| \geq 0$, a Proposição 2.3 item (i), com $n=1$ e $K_{1}=0$, implica que $\bar{w}\left(t ;\left|w_{0}\right|\right)(x) \geq 0$ para todo $t \geq 0$ e todo $x \in \Omega$. Logo $\bar{w}\left(t ;\left|w_{0}\right|\right)(x)$ satisfaz o problema

$$
\begin{aligned}
& \bar{w}_{t}=\Delta \bar{w}+L_{f} \bar{w}+C_{f} \quad \text { em } \quad \Omega, \\
& \frac{\partial \bar{w}}{\partial \bar{n}}=+L_{g} \bar{w}+C_{g}-2 k \bar{w} \quad \text { em } \Gamma,
\end{aligned}
$$

com dado inicial $\left|w_{0}\right| \cdot$ 
Consideremos agora a mudança de variáveis $\tilde{w}=\bar{w}-\phi_{1}$, ou seja, $\bar{w}=\tilde{w}+\phi_{1}$, onde $\phi_{1}$ é a solução do seguinte problema elíptico

$$
\begin{aligned}
& -\Delta \phi_{1}=+L_{f} \phi_{1}+C_{f} \quad \text { em } \quad \Omega, \\
& \frac{\partial \phi_{1}}{\partial \vec{n}}=+L_{g} \phi_{1}+C_{g}-2 k \phi_{1} \quad \text { em } \Gamma .
\end{aligned}
$$

Assim, (3.9) e (3.10) implica que $\tilde{w}\left(t ;\left|w_{0}\right|-\phi_{1}(0)\right)$ é a solução do problema

$$
\begin{aligned}
& \tilde{w}_{t}=\Delta \tilde{w}+L_{f} \tilde{w} \quad \text { em } \quad \Omega, \\
& \frac{\partial \tilde{w}}{\partial \tilde{n}}=+L_{g} \tilde{w}-2 k \tilde{w} \quad \text { em } \Gamma .
\end{aligned}
$$

Para mostrarmos algumas propriedades da solução do problema (3.11), precisamos antes estudarmos seu problema de autovalor associado. Consideremos os seguintes problemas de autovalores:

$$
\left\{\begin{array}{l}
-\Delta w=\mu w \quad \text { em } \quad \Omega \\
\frac{\partial w}{\partial \vec{n}}+\left(2 k-L_{g}\right) w=0 \quad \text { em } \Gamma
\end{array}\right.
$$

e

$$
\left\{\begin{array}{l}
-\Delta w=\mu w \quad \text { em } \quad \Omega \\
w=0 \quad \text { em } \quad \Gamma .
\end{array}\right.
$$

Para utilizarmos os resultados contidos em [11], precisamos colocarmos uma restrição sobre a condição de fronteira em (3.12) dada por

$$
\theta:=2 k-L_{g}>0 \text {. }
$$

Tal restrição é a primeira condição sobre a constante $k$, do problema (3.1), para obtermos a sincronização desejada. O próximo lema pode ser encontrado em [10].

Lema 3.3 Considere $\theta$ como em (3.14), e sejam $\mu_{1}(\theta)$ e $\mu_{\infty}$ respectivamente os primeiros autovalores de (3.12) e (3.13). Então,

$$
\mu_{1}(\theta)=\inf \left\{\int_{\Omega}|\nabla \phi|^{2}+\theta \int_{\Gamma}|\phi|^{2} \mid \phi \in H^{1}(\Omega) \text { e }\|\phi\|_{L^{2}(\Omega)}=1\right\}
$$

Ainda,

$$
\mu_{1}(\theta) \leq \mu_{\infty} \quad e \quad \lim _{\theta \rightarrow+\infty} \mu_{1}(\theta)=\mu_{\infty}
$$


Demonstração: A primeira parte é um resultado bem conhecido, e pode ser encontrado em [11]. Uma consequência imediata deste resultado é que $\mu_{1}\left(\theta_{1}\right) \leq \mu_{1}\left(\theta_{2}\right)$ para $\theta_{1} \leq \theta_{2}$. Também, como $H_{0}^{1}(\Omega) \subset H^{\mathrm{l}}(\Omega)$, segue que para todo $\theta>0$,

$$
\begin{aligned}
\mu_{1}(\theta) & =\inf \left\{\int_{\Omega}|\nabla \phi|^{2}+\theta \int_{\Gamma}|\phi|^{2} \mid \phi \in H^{1}(\Omega) \text { e }\|\phi\|_{L^{2}(\Omega)}=1\right\} \\
& \leq \inf \left\{\int_{\Omega}|\nabla \phi|^{2} \mid \phi \in H_{0}^{1}(\Omega) \text { e }\|\phi\|_{L^{2}(\Omega)}=1\right\} \\
& =\mu_{\infty}
\end{aligned}
$$

Como $\mu_{1}(\theta)$ é crescente e limitada, o limite existe e $\lim _{\theta \rightarrow+\infty} \mu_{1}(\theta) \leq \mu_{\infty}$. Portanto, existem sequências $\left\{\theta_{n}, n \geq 1\right\} \subset \mathbb{R}, \theta_{n} \rightarrow+\infty$ e $\left\{\phi_{n}, n \geq 1\right\} \subset H^{1}(\Omega)$ com $\left\|\phi_{n}\right\|_{L^{2}(\Omega)}=$ 1 , para todo $n \geq 1$ tais que

$$
\int_{\Omega}\left|\nabla \phi_{n}\right|^{2}+\theta_{n} \int_{\Gamma}\left|\phi_{n}\right|^{2} \leq \mu_{\infty}
$$

Daí, $\left\{\phi_{n}, n \geq 1\right\}$ é limitada em $H^{1}(\Omega)$ então, existe uma subsequência, a qual também denotaremos por $\left\{\phi_{n}, n \geq 1\right\}$, e $\phi \in H^{1}(\Omega)$ tal que

$$
\phi_{n} \rightarrow \phi \text { em } H^{1}(\Omega) \text { e } \phi_{n} \rightarrow \phi \text { em } L^{2}(\Omega)
$$

e da mesma forma,

$$
\gamma\left(\phi_{n}\right) \rightarrow \gamma(\phi) \text { em } L^{2}(\Gamma)
$$

onde $\gamma$ denota o operador traço. Assim, $\|\phi\|_{L^{2}(\Omega)}=1$, e como $\theta_{n} \rightarrow \infty$ e $\theta_{n}\left\|\phi_{n}\right\|_{L^{2}(\Gamma)}$ é limitada para todo $n \geq 1$, obtemos que

$$
\int_{\Gamma}\left|\phi_{n}\right|^{2} \rightarrow 0, \text { quando } n \rightarrow+\infty
$$

Então, $\phi \in H_{0}^{1}(\Omega)$. Mas

$$
\int_{\Omega}|\nabla \phi|^{2} \leq \liminf _{n \rightarrow+\infty} \int_{\Omega}\left|\nabla \phi_{n}\right|^{2}
$$

Portanto,

$$
\lim _{n \rightarrow+\infty} \int_{\Omega}\left|\nabla \phi_{n}\right|^{2}+\theta_{n} \int_{\Gamma}\left|\phi_{n}\right|^{2} \geq \int_{\Omega}|\nabla \phi|^{2} \geq \mu_{\infty}
$$


Como o argumento utilizado independe da escolha da sequência escolhida, o resultado segue.

Segue imediatamente do Lema 3.3 que uma condição a priori, para o primeiro autovalor $\mu_{1}(k)$, do problema de autovalor

$$
\left\{\begin{array}{l}
-\Delta \psi-L_{f} \psi=\mu \psi \quad \text { em } \quad \Omega \\
\frac{\partial \psi}{\partial \vec{n}}+\left(2 k-L_{g}\right) \psi=0 \quad \text { em } \Gamma
\end{array}\right.
$$

associado com (3.11), ser positivo é que

$$
L_{f}<\mu_{\infty},
$$

onde $\mu_{\infty}$ é o primeiro autovalor de (3.13). Com isso temos o seguinte resultado:

Teorema 3.1 Para $i=1,2$ sejam $f_{i}, g_{i}: \mathbb{R} \rightarrow \mathbb{R}$ funções localmente lipschtizianas satisfazendo (C1) ou (C2), (2.10) e (3.2). Suponhamos que o primeiro autovalor de (2.11) seja positivo, e que as constantes $L_{f}$ e $L_{g}$ como em (3.3), satisfaçam (3.14) $e$ (3.16). Então, existe uma constante $k_{0}=k_{0}\left(\Omega, f_{1}, f_{2}, g_{1}, g_{2}\right)$ tal que para todo $k>k_{0}$, para quase todo $x \in \Omega$ e para todo $w_{0}=u_{0}-v_{0} \operatorname{com}\left(u_{0}, v_{0}\right) \in L^{q}\left(\Omega, \mathbb{R}^{2}\right)$ ou $\left(u_{0}, v_{0}\right) \in$ $W^{1, q}\left(\Omega, \mathbb{R}^{2}\right)$, respectivamente temos

$$
\limsup _{t \rightarrow \infty} \bar{w}\left(t ;\left|w_{0}\right|\right)(x) \leq\left\|\phi_{1}\right\|_{L^{\infty}(\Omega)}
$$

onde $\phi_{1}$ é a solução do problema elíptico (3.10).

Demonstração: Tomando $k$ satisfazendo (3.14) e (3.16), é fácil ver que

$$
\left\|\tilde{w}\left(t ;\left|w_{0}\right|-\phi_{1}(0)\right)\right\|_{L^{\infty}(\Omega)} \rightarrow 0, \text { quando } t \rightarrow+\infty,
$$

onde $\tilde{w}\left(t ;\left|w_{0}\right|-\phi_{1}(0)\right)$ é a solução do problema (3.11).

Mas, como $\bar{w}\left(t ;\left|w_{0}\right|\right)=\tilde{w}\left(t ;\left|w_{0}\right|-\phi_{1}(0)\right)+\phi_{1}$ e $\bar{w}\left(t ;\left|w_{0}\right|\right) \geq 0$, a prova do teorema está completa.

De forma análoga temos o 
Teorema 3.2 Suponhamos que todas as hipóteses do Teorema 3.1 estejam satisfeitas, $e$ seja $k_{0}$ a constante encontrada no referido teorema. Então, para todo $k>k_{0}$, para quase todo $x \in \Omega$ e para todo $w_{0}=u_{0}-v_{0} \operatorname{com}\left(u_{0}, v_{0}\right) \in L^{q}\left(\Omega, \mathbb{R}^{2}\right)$ ou $\left(u_{0}, v_{0}\right) \in W^{1, q}\left(\Omega, \mathbb{R}^{2}\right)$, respectivamente temos

$$
\liminf _{t \rightarrow \infty} \underline{w}\left(t ;-\left|w_{0}\right|\right)(x) \geq-\left\|\phi_{2}\right\|_{L^{\infty}(\Omega)},
$$

onde $\phi_{2}$ é a solução do seguinte problema elíptico

$$
\begin{aligned}
& -\triangle \phi_{2}=+L_{f} \phi_{2}-C_{f} \quad \text { em } \quad \Omega, \\
& \frac{\partial \phi_{2}}{\partial \vec{n}}=+L_{g} \phi_{2}-C_{g}-2 k \phi_{2} \quad \text { em } \Gamma .
\end{aligned}
$$

Observemos que $\phi_{2}=-\phi_{1}$, onde $\phi_{1}$ e $\phi_{2}$ são soluções de (3.10) e (3.17) respectivamente. Portanto, os Lemas 3.1 e 3.2 e os Teoremas 3.1 e 3.2 implicam que,

$$
\underline{w}\left(t ;-\left|w_{0}\right|\right)(x) \leq w\left(t ; w_{0}\right)(x) \leq \bar{w}\left(t ;\left|w_{0}\right|\right)(x)
$$

$-\left\|\phi_{1}\right\|_{L^{\infty}(\Omega)} \leq \liminf _{t \rightarrow \infty} w\left(t ; w_{0}\right)(x) \leq \limsup \sin _{t \rightarrow \infty} w\left(t ; w_{0}\right)(x) \leq\left\|\phi_{1}\right\|_{L^{\infty}(\Omega)}$,

para todo $x \in \Omega$ e para todo $w_{0}=u_{0}-v_{0}$ com $u_{0}, v_{0} \in X$, onde $X=L^{q}\left(\Omega, \mathbb{R}^{2}\right)$ ou $X=W^{1, q}\left(\Omega, \mathbb{R}^{2}\right)$.

O próximo resultado, é uma adaptação do Lema 8.1 item (iii) de [6], precisamos antes do seguinte lema técnico:

Lema 3.4 Seja u(x) uma função somável em $\Omega$. Suponhamos que para $r \geq r_{0}>0$, tenhamos

$$
\int_{A_{r}}(u(x)-r) d x \leq \gamma r^{\alpha}\left|A_{r}\right|^{1+\varepsilon}
$$

onde $\gamma, \alpha$ e $\varepsilon$ são constantes tais que $\varepsilon>0$ e $0 \leq \alpha \leq I+\varepsilon, e$

$$
A_{r}:=\{x \in \Omega \mid u(x)>r\} \text {. }
$$

Então,

$$
\operatorname{supess}(u) \leq K
$$

onde $K=K\left(\gamma, \alpha, \varepsilon, r_{0},\|u\|_{L^{1}\left(A_{r_{0}}\right)}\right)$, e "supess" denota o supremo essencial. 
Demonstração: A prova desse lema pode ser encontrada em [18], página 71.

Proposição 3.1 Suponhamos que todas as hipóteses do Teorema 3.1 estejam satisfeitas. Então, existe uma constante $k_{0}=k_{0}\left(\Omega, f_{1}, f_{2}, g_{1}, g_{2}\right)$ tal que para todo $k \geq k_{0}$,

$$
\left\|\phi_{1}\right\|_{L^{\infty}(\Omega)} \leq C\left(\left|C_{f}\right|+\left|C_{g}\right|\right)
$$

onde $C=C\left(\Omega, f_{1}, f_{2}, g_{1}, g_{2}\right)>0$ e $\phi_{1}$ é soluçäo de (3.10).

Demonstração: Mostraremos primeiramente uma limitação, uniforme em $k$, para a norma $L^{2}(\Omega)$ da solução do problema (3.10).

As restrições feitas sobre $k$ implicam que $L_{k}:=2 k-L_{g}>0$. Assim, multiplicando a primeira equação de (3.10) por $\phi_{1}$ e integrando por partes obtemos que

$$
\int_{\Omega}\left|\nabla \phi_{1}\right|^{2}-L_{f} \int_{\Omega} \phi_{1}^{2}+L_{k} \int_{\Gamma} \phi_{1}^{2}-C_{f} \int_{\Omega} \phi_{1}-C_{g} \int_{\Gamma} \phi_{1}=0
$$

Tomando $\phi=\phi_{1}-\frac{C_{g}}{2 L_{k}}$, e usando o fato que o primeiro autovalor, $\lambda_{1}(k)$, de (3.15) ser positivo, (3.19) implica que

$$
\lambda_{1}(k) \int_{\Omega} \phi^{2}+C_{1} \int_{\Omega} \phi+C_{2} \leq 0
$$

onde $C_{1}:=-\left(C_{f}+\frac{L_{f} C_{g}}{L_{k}}\right)$ e $C_{2}:=-\left(|\Gamma| \frac{C_{g}^{2}}{4 L_{k}}+|\Omega|\left(\frac{3 C_{g}^{2} L_{f}}{4 L_{k}^{2}}+\frac{C_{f} C_{g}}{2 L_{k}}\right)\right)$.

Segue, da Desigualdade de Young $\operatorname{com} \varepsilon=\frac{\left|C_{1}\right|}{\lambda_{1}(k)}$, que

$$
\|\phi\|_{L^{2}(\Omega)} \leq \frac{1}{\sqrt{\lambda_{1}(k)}} \sqrt{2\left|C_{2}\right|+\frac{\left|C_{1}\right|^{2}|\Omega|}{\sqrt{\lambda_{1}(k)}}} .
$$

Portanto,

$$
\left\|\phi_{1}\right\|_{L^{2}(\Omega)} \leq \frac{1}{\sqrt{\lambda_{1}(k)}} \sqrt{2\left|C_{2}\right|+\frac{\left|C_{1}^{2}\right| \Omega \mid}{\sqrt{\lambda_{1}(k)}}}+\frac{C_{g}|\Omega|^{1 / 2}}{2 L_{k}} .
$$

Agora, o Lema 3.3, e as expressōes de $C_{1}$ e $C_{2}$ implicam que, para $k>0$ suficientemente grande, existe uma constante $\bar{C}=C\left(|\Omega|,|\Gamma|, f_{1}, f_{2}, g_{1}, g_{2}\right)$ tal que

$$
\left\|\phi_{1}\right\|_{L^{2}(\Omega)} \leq \bar{C}\left(\left|C_{f}\right|+\left|C_{g}\right|\right)
$$


Vamos agora obter estimativas em $L^{\infty}(\Omega)$ para $\phi_{1}$. Em (3.10) tomemos $k_{0}>0$ tal que $2 k_{0}-L_{g}=L_{g}$, então para todo $k \geq k_{0}$ temos $2 k-L_{g} \geq L_{g}$. Defina

$$
r_{0}:=\max \left\{\frac{C_{g}}{L_{g}}, 1\right\}
$$

e para todo $r \geq r_{0}$ seja $\phi:=\left(\phi_{1}-r\right)^{+}$, considere também

$$
A_{r}:=\left\{x \in \Omega: \phi_{1}(x)>r\right\}
$$

onde $\phi_{1}$ é a soluçāo do problema elíptico (3.10).

Multiplicando a primeira equação de (3.10) por $\phi$, integrando por partes e usando a definição de $\phi$ segue que

$$
\int_{\Omega}|\nabla \phi|^{2}+\left(2 k-L_{g}\right) \int_{\Gamma} \phi^{2}-L_{f} \int_{\Omega} \phi^{2}=\int_{\Omega}\left(C_{f}+L_{f} r\right) \phi+\int_{\Gamma}\left(C_{g}-\left(2 k-L_{g} r\right)\right) \phi .
$$

A definições de $r_{0}$ e $\phi$ implicam que

$$
\int_{\Omega}|\nabla \phi|^{2}+\left(2 k-L_{g}\right) \int_{\Gamma} \phi^{2}-L_{f} \int_{\Omega} \phi^{2} \leq \int_{\Omega}\left(C_{f}+L_{f} r\right) \phi
$$

Usando o fato do primeiro autovalor, $\lambda_{1}(k)$, de (3.15) ser positivo obtemos, para todo $k>k_{0}$ satisfazendo as condiçōes do Teorema (3.1), que

$$
\begin{aligned}
\min \left\{1, L_{g}\right\}\left(\int_{\Omega}|\nabla \phi|^{2}+\int_{\Gamma} \phi^{2}\right) & \leq \int_{\Omega}|\nabla \phi|^{2}+\left(2 k-L_{g}\right) \int_{\Gamma} \phi^{2} \\
& \leq \frac{\left(1+L_{f}\right)\left(C_{f}+L_{f} r\right)}{\lambda_{1}(k)} \int_{\Omega} \phi .
\end{aligned}
$$

Denotando $M:=\frac{\left(1+L_{f}\right)\left(C_{f}+L_{f} r\right)}{\lambda_{1}(k) \min \left\{1, L_{g}\right\}}$, obtemos que

$$
\int_{\Omega}|\nabla \phi|^{2}+\int_{\Gamma} \phi^{2} \leq M \int_{\Omega}|\phi|
$$

Agora, uma desigualdade devido a Maz'ja [19], implica que existe uma constante $C_{3}=C_{3}(|\Omega|, N)$ tal que

$$
\begin{aligned}
& \|\phi\|_{L}^{2}{ }_{L}^{\frac{2 N}{N-1}(\Omega)} \leq C_{3}\left(\int_{\Omega}|\nabla \phi|^{2}+\int_{\Gamma} \phi^{2}\right) \leq C_{3} M \int_{\Omega}|\phi| \\
& \leq C_{3} M|\Omega|^{1 / p}\|\phi\|_{L^{p^{\prime}}(\Omega)} \\
& \leq C_{3} M|\Omega|^{1 / p}\|\phi\|_{L^{\frac{2 N}{N-1}(\Omega)}}\left|A_{r}\right|^{1 / 2-1 / p+1 / N} \text {. }
\end{aligned}
$$

Portanto,

$$
\|\phi\|_{L^{2 N} \frac{2 N}{N-1}} \leq C_{3} M|\Omega|^{1 / p}\left|A_{r}\right|^{1 / 2-1 / p+1 / N} .
$$


Tomando $p=2 N$, obtemos, do Lema 3.4, que

$$
\max \left\{\phi_{1}(x), x \in \Omega\right\} \leq C_{3} M|\Omega|^{1 / 2 N} r_{0}\left\|\phi_{1}\right\|_{L^{1}\left(A_{r_{0}}\right)} \frac{N+1}{N} .
$$

Trocando $\phi_{1}$ por $-\phi_{1}$ obtemos que

$$
\left\|\phi_{1}\right\|_{L^{\infty}(\Omega)} \leq C_{3} M|\Omega|^{1 / 2 N} r_{0} \frac{N+1}{N}\left\|\phi_{1}\right\|_{L^{1}\left(A_{r_{0}}\right)} .
$$

Usando a primeira parte da prova temos que

$$
\left\|\phi_{1}\right\|_{L^{\infty}(\Omega)} \leq C_{3} M|\Omega|^{1 / 2 N} r_{0} \frac{N+1}{N} \bar{C}\left(\left|C_{f}\right|+\left|C_{g}\right|\right),
$$

a s expressões de $M$ e de $C_{3}$ implicam que existe uma contante $C=C\left(\Omega,|\Gamma|, N, f_{1}\right.$, $\left.f_{2}, g_{1}, g_{2}\right)$ tal que

$$
\left\|\phi_{1}\right\|_{L^{\infty}(\Omega)} \leq C\left(\left|C_{f}\right|+\left|C_{g}\right|\right)
$$

para $k$ suficientemente grande.

Finalmente temos 0

Corolário 3.1 Suponhamos que todas as hipóteses do Teorema 3.1 estejam satisfeitas. Então, para todo $\left(u_{0}, v_{0}\right) \in L^{q}\left(\Omega, \mathbb{R}^{2}\right)$ ou $\left(u_{0}, v_{0}\right) \in W^{1, q}\left(\Omega, \mathbb{R}^{2}\right)$, existe uma constante $k_{0}=k_{0}\left(\Omega, f_{1}, f_{2}, g_{1}, g_{2}\right)$ tal que para $k \geq k_{0}$,

$$
\limsup _{t \rightarrow \infty}\left\|u\left(t ; u_{0}\right)-v\left(t ; v_{0}\right)\right\|_{L^{\infty}(\Omega)} \leq C\left(\left|C_{f}\right|+\left|C_{g}\right|\right),
$$

onde $C_{f}$ e $C_{g}$ são dados em (3.2) e $C \geq 0$ é a constante da Proposição 3.1. Ainda mais, se $f_{1}=f_{2}$ e $g_{1}=g_{2}$ então,

$$
\lim _{t \rightarrow \infty}\left\|u\left(t ; u_{0}\right)-v\left(t ; v_{0}\right)\right\|_{L^{\infty}(\Omega)}=0
$$

para todo $k>k_{0}$.

\subsection{Um Exemplo}

Nesta seção, para um conjunto $\Omega \subset \mathbb{R}$ específicos e funções $f$ e $g$ satisfazendo as condições das seções anteriores, calcularemos de forma precisa o valor da constante $k_{0}=$ $k_{0}(\Omega, f, g)$ encontrada na Seção 4 . Faremos o exemplo em $L^{2}\left(\Omega, \mathbb{R}^{2}\right)$ por simplicidade. 
Seja $\Omega=(0,1)$ e considere o problema

$$
\left\{\begin{array}{l}
u_{t}(t, x)=\Delta u(t, x)+f(u(t, x)) \quad t>0, \quad x \in \Omega, \\
v_{t}(t, x)=\Delta v(t, x)+f(v(t, x)) \quad t>0, \quad x \in \Omega, \\
\frac{\partial u}{\partial \vec{n}}(t, x)=g(u(t, x))-k(u(t, x)-v(t, x)) \quad t>0, \quad x \in \Gamma, \\
\frac{\partial v}{\partial \vec{n}}(t, x)=g(v(t, x))+k(u(t, x)-v(t, x)) \quad t>0, \quad x \in \Gamma,
\end{array}\right.
$$

Os resultados obtidos nas seções anteriores, implicam que para obter sicronização para o problema (3.21), basta mostrar que o primeiro autovalor, $\mu_{1}(k)$, associado ao problema

$$
\left\{\begin{array}{l}
w_{t}=\Delta w+L_{f} w \quad \text { em } \quad \Omega, \\
w_{x}-\left(2 k-L_{g}\right) w=0 \quad \text { em } \quad x=0 \\
w_{x}+\left(2 k-L_{g}\right) w=0 \quad \text { em } \quad x=1,
\end{array}\right.
$$

é positivo. Consideremos

$$
\theta=2 k-L_{g}
$$

A primeira restrição a ser feita, é que $\theta$ deve ser positivo, ou seja $k>\frac{L_{g}}{2}$.

Consideremos o operador $A_{\theta}: D\left(A_{\theta}\right) \subset L^{2}(\Omega) \rightarrow L^{2}(\Omega)$, definido por

$$
\begin{aligned}
& D\left(A_{\theta}\right)=\left\{\phi \in H^{2}(\Omega): \phi_{x}(0)=\theta \phi(0) \text { e } \phi_{x}(1)=-\theta \phi(1)\right\} \\
& A_{\theta}(\phi)=-\phi_{x x}
\end{aligned}
$$

e considere o seguinte problema de autovalor $A_{\theta} \phi=\mu \phi$. Se $\mu=\gamma^{2}$ então, $\gamma$ é dado por

$$
\operatorname{cotg} \gamma=G\left(\frac{\gamma}{\theta}\right)
$$

onde $G(s)=\frac{1}{2}\left(\frac{\gamma}{\theta}-\frac{\theta}{\gamma}\right)$, para detalhes veja [16].

Observemos que fixado $0<\gamma<\pi$, temos

$$
\lim _{\theta \rightarrow+\infty} G\left(\frac{\gamma}{\theta}\right)=-\infty
$$


Agora, dado $L_{f} \in\left(0, \pi^{2}\right)$, existe $\theta_{0}>0$ tal que

$$
\operatorname{cotg} \sqrt{L_{f}}=\frac{1}{2}\left(\frac{\sqrt{L_{f}}}{\theta_{0}}-\frac{\theta_{0}}{\sqrt{L_{f}}}\right),
$$

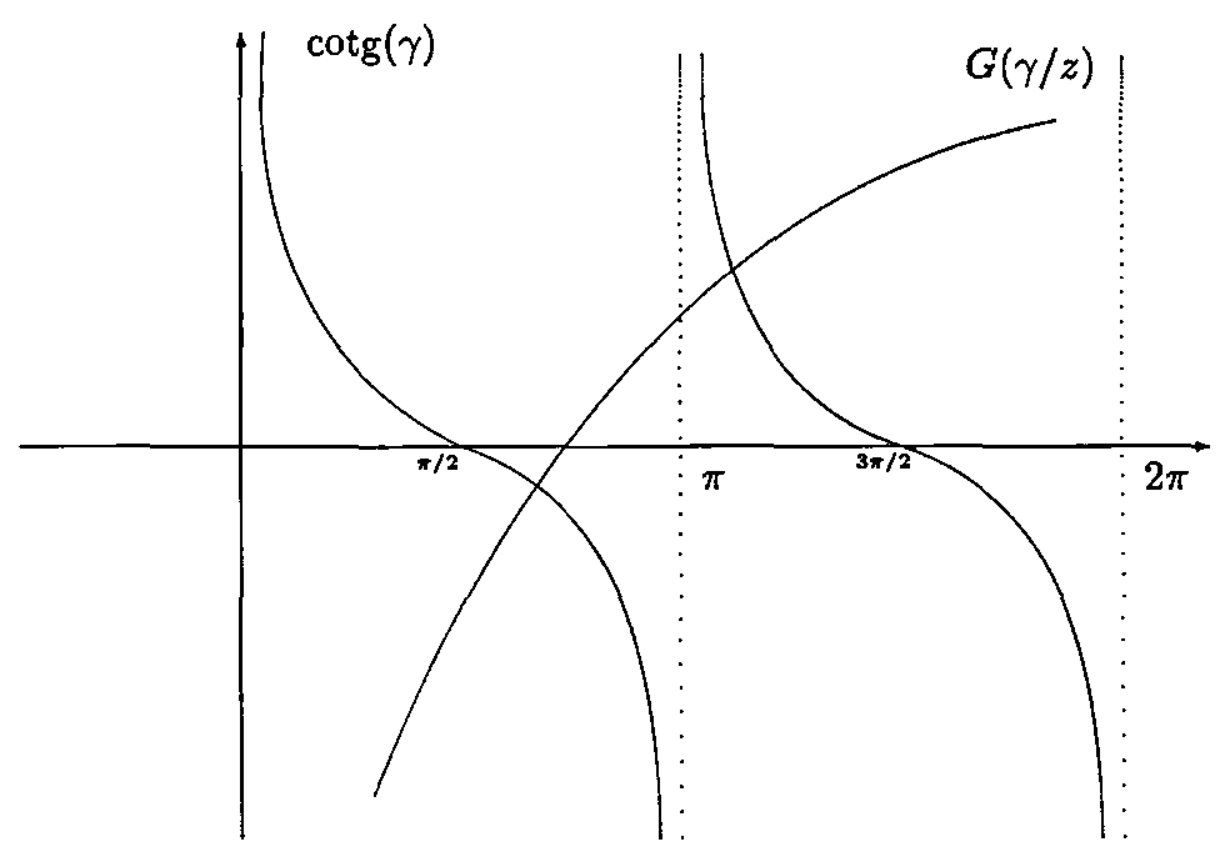

Figura 3.1: Gráfico de $G(\gamma / z)$

observe que $\theta_{0}$ é dado por

$$
\theta_{0}=-\operatorname{cotg}\left(\sqrt{L_{f}}\right) \sqrt{L_{f}}+\sqrt{L_{f}} \sqrt{\left(\operatorname{cotg}\left(\sqrt{L_{f}}\right)\right)^{2}+1} .
$$

Assim, para todo $\theta>\theta_{0}$, ou seja, para

$$
k>\frac{L_{g}}{2}+\frac{\theta_{0}}{2}:=k_{0}(\Omega, f, g),
$$

o primeiro autovalor, $\mu_{1}(k)$, associado ao problema (3.22), é positivo. Portanto, o Corolário 3.1 implica que para todo $k>k_{0}(\Omega, f, g)$,

$$
\lim _{t \rightarrow+\infty}\left\|u\left(t ; u_{0}\right)-v\left(t ; v_{0}\right)\right\|_{L^{\infty}(\Omega)}=0
$$

para todo $\left(u_{0}, v_{0}\right) \in L^{q}\left(\Omega, \mathbb{R}^{2}\right)$, já que as constantes $C_{f}$ e $C_{g}$ dadas em (3.2) são nulas. 


\section{Capítulo 4}

\section{Homogeneização em Problemas Parabólicos com Condição de \\ Fronteira não Linear}

Seja $\Omega \subset \mathbb{R}^{N}, N \in \mathbb{N}$, um domínio limitado com fronteira $\Gamma:=\partial \Omega$ suave. Consideremos o problema

$$
\left\{\begin{array}{l}
u_{t}(t, x)=D \Delta u(t, x)+F(u(t, x)) \quad t>0, \quad x \in \Omega, \\
D \frac{\partial u}{\partial \vec{n}}(t, x)=G(u(t, x)) \quad t>0, \quad x \in \Gamma
\end{array}\right.
$$

onde

- $u=\left(u_{1}, u_{2}, \cdots, u_{n}\right)^{T}, n \geq 1$

- $\frac{\partial u}{\partial \vec{n}}=\langle\nabla u, \vec{n}\rangle, \vec{n}$ é a normal à $\Gamma$, apontando para fora de $\Omega$;

- $D$ é a matriz, $n \times n$, com $d_{i}>0$, para todo $i=1,2, \cdots, n$;

- $F=\left(F_{1}, F_{2}, \cdots, F_{n}\right)^{T}: \mathbb{R}^{n} \rightarrow \mathbb{R}^{n}$ e $G=\left(G_{1}, G_{2}, \cdots, G_{n}\right)^{T}: \mathbb{R}^{n} \rightarrow \mathbb{R}^{n}$ são funções localmente lipschitzianas, onde $F_{i}, G_{i}, i=1,2, \cdots, n$, são definidas em (2.8) $\operatorname{com} c=0$, e satisfazendo todas as condiçōes dos Capítulos 1 e 2. 
Recordemos que o problema (4.1) possui uma única solução globalmente definida, dada pela fórmula da variação das constantes

$$
T_{D}(t) u_{0}=e^{-A_{D} t} u_{0}+\int_{0}^{t} e^{-A_{D}(t-s)} H\left(T_{D}(s) u_{0}\right) d s,
$$

para $u_{0} \in X$, onde $X=L^{q}\left(\Omega, \mathbb{R}^{n}\right)$ ou $X=W^{1, q}\left(\Omega, \mathbb{R}^{n}\right), A_{D}=\operatorname{diag}\left(A_{1}, A_{2}, \cdots, A_{n}\right)$ é definido, para $i=1,2, \cdots, n$, por

$$
\begin{aligned}
& D\left(A_{i}\right)=\left\{\phi \in W^{2, q}(\Omega, \mathbb{R}) ; \frac{\partial \phi}{\partial \vec{n}}=0\right\}, \\
& A_{i} \phi=-d_{i} \Delta \phi
\end{aligned}
$$

e a função $H:=F_{\Omega}+G_{\Gamma}$, atuando em funções testes, $\phi$, adequadas da seguinte forma:

$$
\langle H(u), \phi\rangle=\left\langle F_{\Omega}(u), \phi\right\rangle+\left\langle G_{\Gamma}(u), \phi\right\rangle=\int_{\Omega} F(u(x)) \cdot \phi(x)+\int_{\Gamma} G(u(x)) \cdot \phi(x) .
$$

O objetivo principal deste capítulo é mostrar que existe uma vizinhança, $\mathcal{N}\left(\mathcal{A}_{0}\right)$, de $\mathcal{A}_{0}$ em $\mathbb{R}^{n}$ tal que

$$
\mathcal{A}_{X}(D) \subset \mathcal{N}\left(\mathcal{A}_{0}\right)
$$

para $d:=\min \left\{d_{1}, \cdots, d_{n}\right\}$ suficientemente grande, onde $\mathcal{A}_{X}(D)$ é o atrator global compacto para o problema (4.1), encontrado no Teorema 2.8 e $\mathcal{A}_{0}$ é o atrator global da equação diferencial ordinária limite:

$$
\dot{v}(t)=F(v(t))+|\Gamma| G(v(t)) .
$$

Tal propriedade é chamada de homogeneização. Os resultados desta seção generalizam parcialmente os resultados de $[13,15]$.

\subsection{Enunciado dos Resultados Principais}

Nesta seçāo vamos introduzir algumas notações e conceitos, enunciaremos também o principal resultado deste capítulo. Ao longo deste capítulo, $X=L^{q}\left(\Omega, \mathbb{R}^{n}\right)$ ou $X=W^{1, q}\left(\Omega, \mathbb{R}^{n}\right)$.

Para $i=1,2, \cdots, n$, sejam $\lambda_{i}(D)$ o primeiro autovalor associado ao operador definido em (4.3). Sabemos que $\lambda_{i}(D)=0, i=1,2, \cdots, n$. Consideremos $\varphi_{i}(D)$, $i=1,2, \cdots, n$, a correspondente autofunção normalizada. Neste caso temos que

$$
\varphi_{i}(D)=|\Omega|^{-\frac{1}{2}}
$$


$i=1,2, \cdots, n$. Podemos, sem perda da generalidade, supor que $|\Omega|=1$, então $\varphi_{i}(D)=$ $1, i=1,2, \cdots, n$. Definamos

$$
\Phi_{D}:=\left[\begin{array}{ccccc}
1 & 0 & 0 & \cdots & 0 \\
0 & 1 & 0 & \cdots & 0 \\
0 & 0 & 1 & \cdots & 0 \\
\vdots & \vdots & \vdots & \ddots & \vdots \\
0 & 0 & 0 & \cdots & 1
\end{array}\right]_{n \times n}
$$

Agora, com a notação da Subseção 1.1.7, fixemos $q \in(1, \infty)$ e $E^{\alpha}:=E_{q}^{\alpha}, \alpha \in$ $[-2,2]$. Consideremos a seguinte decomposição de $E^{\alpha}$ :

$$
E^{\alpha}=U \oplus U_{\alpha}^{\perp}
$$

onde

$$
\begin{aligned}
& U=\left\{\varphi=\Phi(D) v ; \quad v \in \mathbb{R}^{n}\right\} \equiv \mathbb{R}^{n}, \\
& U_{\alpha}^{\perp}=\left\{\varphi \in E^{\alpha} ; \quad(\phi, \varphi\rangle=0, \quad \psi \in U\right\}
\end{aligned}
$$

e

$$
\langle\psi, \varphi\rangle=\int_{\Omega} \varphi(x)^{T} \psi(x) d x, \quad \psi \in U, \varphi \in E^{\alpha} .
$$

Observemos que se $\psi \in U$, então $\psi \in L^{\infty}\left(\Omega, \mathbb{R}^{n}\right)$ e, portanto, a integral acima fica bem definida para toda $\varphi \in E^{\alpha}$. Notemos também que $U$ pode ser visto como um subespaço $n$-dimensional de $E^{\alpha}$, conténdo apenas funções constantes.

Seja $u(t, \cdot):=T_{D}(t) u_{0}, u_{0} \in X$, a solução de (4.1) em $X$. Podemos escrever

$$
u(t, x)=v(t)+w(t, x), \quad x \in \Omega,
$$

onde $v(t) \in U \equiv \mathbb{R}^{n}$ e $w(t, \cdot) \in U_{\alpha}^{\perp}, t>0$. Integrando (4.6) obtemos que

$$
\begin{aligned}
\int_{\Omega} u(t, x) d x & =v(t)+\int_{\Omega} w(t, x) d x \\
& =v(t)
\end{aligned}
$$

pois $1 \in U$. Assim,

$$
v(t)=\int_{\Omega} u(t, x) d x \text { e } w(t, x)=u(t, x)-v(t), \quad t>0, x \in \Omega .
$$


Logo,

$$
\begin{aligned}
\frac{d}{d t} v(t) & =\frac{d}{d t}\left(\int_{\Omega} u(t, x) d x\right) \\
& =\int_{\Omega} D \Delta u(t, x) d x+\int_{\Omega} F(u(t, x)) d x \\
& =\int_{\Omega} F(u(t, x)) d x+\int_{\Gamma} G(u(t, x)) d x \\
& =\int_{\Omega} F(v(t)+w(t, x)) d x+\int_{\Gamma} G(v(t)+w(t, x)) d x \\
& =P(v(t), w(t, \cdot)),
\end{aligned}
$$

onde, para $v \in \mathbb{R}^{n}$ e $w \in U_{\alpha}^{\perp}$

$$
P(v, w)=\int_{\Omega} F(v+w(x)) d x+\int_{\Gamma} G(v+w(x)) d x .
$$

Ainda, para $t>0$ e $x \in \Omega$ temos

$$
\begin{aligned}
\frac{\partial}{\partial t} w(t, x) & =\frac{\partial}{\partial t}(u(t, x)-v(t)) \\
& =D \Delta u(t, x)+F(u(t, x))-(P(v(t), w(t, \cdot)) \\
& =D \Delta w(t, x)+F(u(t, x))-(P(v(t), w(t, \cdot)) .
\end{aligned}
$$

Também, para $x \in \Gamma$ e $t>0$,

$$
\begin{aligned}
D \frac{\partial}{\partial \vec{n}} w(t, x) & =D \frac{\partial}{\partial \vec{n}} u(t, x) \\
& =G(v(t)+w(t, x)) .
\end{aligned}
$$

Portanto, usando a decomposição (4.5), podemos escrever toda solução de (4.1) como uma solução do problema

$$
\left\{\begin{array}{l}
\frac{d}{d t} v(t)=P(v(t), w(t, \cdot)), \quad t>0 \\
\frac{\partial}{\partial t} w(t, x)=D \Delta w(t, x)+Q(v(t), w(t, x)), \quad t>0, x \in \Omega \\
D \frac{\partial w}{\partial \vec{n}}=G(v(t)+w(t, x)) \quad t>0, x \in \Gamma
\end{array}\right.
$$


onde

$$
\begin{aligned}
& P: U+U_{\alpha}^{\perp} \rightarrow U \\
& Q: U+U_{\alpha}^{\perp} \rightarrow L^{q}\left(\Omega, \mathbb{R}^{n}\right),
\end{aligned}
$$

são definidos, para $v \in \mathbb{R}^{n}$ e $w \in U_{\alpha}^{\perp}$, por

$$
\begin{aligned}
& P(v, w)=\int_{\Omega} F(v+w(x)) d x+\int_{\Gamma} G(v+w(x)) d x, \\
& (Q(v, w))(x)=\left(F_{\Omega}(v+w)\right)(x)-P(v, w), x \in \Omega .
\end{aligned}
$$

Associamos então com (4.7), a seguinte equação diferencial ordinária limite

$$
\dot{v}(t)=F(v(t))+|\Gamma| G(v(t)) .
$$

Antes de enunciarmos o principal resultado deste capítulo, precisamos da seguinte definição:

Definição 4.1 Sejam $X$ e $Y$ espaços de Banach, $A$ um operador setorial em $X$ e $X^{\alpha}$, $\alpha \in \mathbb{R}$, a escala de espaços de Banach associadas ao operador $A$, como construída na Seção 1.1. Suponhamos que $f: X^{\alpha} \times Y \rightarrow X$ e $g: X^{\alpha} \times Y \rightarrow Y$ são funções localmente lipschitzanas. Diremos que um conjunto $S \subset X^{\alpha} \times Y$ é uma variedade invariante para a equação diferencial

$$
\begin{aligned}
& \dot{x}=-A x+f(x, y) \\
& \dot{y}=g(x, y),
\end{aligned}
$$

se existir uma função $\sigma: Y \rightarrow X^{\alpha}$ tal que para

$$
\left(x_{0}, y_{0}\right) \in S:=\left\{(x, y) \in X^{\alpha} \times Y: x=\sigma(y)\right\},
$$

existe uma solução $(x(\cdot), y(\cdot))$ da equação diferencial acima, com $(x(t), y(t)) \in S$, enquanto a solução estiver definida. Ainda, diremos que a variedade invariante $S$ é exponencialmente atratora se existirem constantes positivas $\gamma$ e $K$ tais que

$$
\|x(t)-\sigma(y(t))\|_{X^{\alpha}} \leq K e^{-\gamma t}\|x(0)-\sigma(y(0))\|_{X^{\alpha}},
$$

enquanto a solução estiver definida. 
Temos assim o:

Teorema 4.1 Sejam $\mathcal{A}_{0}$ o atrator global da $\mathrm{EDO}(4.9)$ e $V \subset \mathbb{R}^{n}$ uma vizinhança de $\mathcal{A}_{0}$ tal que

$$
w(V) \subset \mathcal{A}_{0}
$$

Então para toda vizinhança, W, de $\mathcal{A}_{0}$ tal que

$$
\bar{W} \subset V,
$$

existe $d_{0}>0$ e uma função

$$
\sigma_{d}: \bar{W} \rightarrow U_{\alpha}^{\perp}
$$

para todo $d>d_{0}$, tal que

$$
\sigma_{d}(v) \rightarrow 0, \quad \text { quando } d \rightarrow \infty,
$$

uniformente em $v \in \bar{W} . O$ conjunto

$$
M_{d}=\left\{u=v+\sigma_{d}(v), v \in W\right\}
$$

é uma variedade invariante, exponencialmente atratora para (4.1). Ainda mais, o fluxo sobre esta variedade é dado por

$$
u(t, x)=v(t)+\sigma_{d}(v(t))(x)
$$

onde

$$
\frac{d}{d t} v(t)=P\left(v(t), \sigma_{d}(v(t))\right)
$$

\subsection{Prova do Teorema 4.1}

Nesta seção provaremos o Teorema 4.1. Seja $\Omega \subset \mathbb{R}^{N}$ um subconjunto aberto, limitado e com fronteira, $\Gamma:=\partial \Omega$, suave. A equação

$$
\begin{cases}\frac{\partial}{\partial t} w=D \Delta w, & \text { em } \Omega \\ D \frac{\partial w}{\partial \vec{n}}=0 & \text { em } \Gamma\end{cases}
$$


onde $D$ é a matriz definida em (1.2), gera um semigrupo analítico, $\left\{e^{-A_{D} t} ; t \geq 0\right\}$, em $E^{\alpha}$, com $A_{D}$ definido na Seçāo 4.1, satisfazendo as seguintes estimativas

$$
\begin{aligned}
& \left\|e^{-A_{D} t} w\right\|_{E_{q}^{\alpha}} \leq K_{1} e^{-(d \delta) t}\|w\|_{E_{q}^{\alpha}}, w \in U_{\alpha}^{\perp}, t \geq 0 \\
& \left\|e^{-A_{D} t} w\right\|_{E_{q}^{\alpha}} \leq K_{1} e^{-(d \delta) t} t^{-(\alpha+r)}\|w\|_{E_{q}^{-r}}, w \in U_{-r}^{\perp}, t>0,
\end{aligned}
$$

onde $K_{1}>0, \delta>0$,

$$
d:=\min \left\{d_{1}, d_{2}, \cdots, d_{n}\right\},
$$

$q \in(1, \infty), \alpha$ e $r$ são tomados de tal forma que

$$
2 r>\frac{1}{q^{\prime}} \text { e } \alpha+r<1 .
$$

Observemos que estas restrições implicam que

$$
\alpha<\frac{1}{2}+\frac{1}{2 q} \text {. }
$$

Mostremos inicialmente um resultado que garante uma estimativa do atrator global compacto, $\mathcal{A}_{X}(D)$, do problema (4.1) uniforme em relação à matriz $D$, desde que $d_{i}>1$, $i=1,2, \cdots, n$. A idéia é usar o item (ii) do Teorema 2.8. Comecemos com o

Lema 4.1 Sejam $a \geq 1$ e $\lambda_{1}(a)$ o primeiro autovalor do problema

$$
\begin{array}{ll}
-a \Delta u+C_{0} u=\lambda u & \text { em } \Omega, \\
a \frac{\partial u}{\partial \vec{n}}+B_{0} u=0 & e m \Gamma .
\end{array}
$$

Então

$$
\lambda_{1}(a) \geq \lambda_{1}>0,
$$

onde $\lambda_{1}$ é o primeiro autovalor do problema acima com $a=1$.

Demonstração: Suponhamos que existam constantes $C_{0}, B_{0} \in \mathbb{R}$ satisfazendo (2.10) tais que o primeiro autovalor, $\lambda_{1}$, do problema

$$
\begin{aligned}
& -\triangle u+C_{0} u=\lambda u \text { em } \Omega \text {, } \\
& \frac{\partial u}{\partial \vec{n}}+B_{0} u=0 \quad \text { em } \Gamma \text {, }
\end{aligned}
$$

seja positivo. 
Mas para toda $\phi \in H^{1}(\Omega)$, com $\|\phi\|_{L^{2}(\Omega)}=1$, temos

$$
\int_{\Omega} a|\nabla \phi|^{2}+C_{0} \int_{\Omega}|\phi|^{2}+B_{0} \int_{\Gamma}|\phi|^{2} \geq \int_{\Omega}|\nabla \phi|^{2}+C_{0} \int_{\Omega}|\phi|^{2}+B_{0} \int_{\Gamma}|\phi|^{2},
$$

pois $a \geq 1$. Logo, os resultados de Courant-Hilbert, página 399, implicam que

$$
\lambda_{1}(a) \geq \lambda_{1}>0
$$

o que demonstra o lema.

Uma consequência imediata do Lema 4.1 , é que se $d_{i} \geq 1, i=1,2, \cdots, n$, então o primeiro autovalor, $\lambda_{1}(D)$, de (2.22) satisfaz

$$
\lambda_{1}(D) \geq \lambda_{1}>0,
$$

onde $\lambda_{1}$ é o primeiro autovalor de (4.14). Ainda mais, as constantes $C_{0}$ e $B_{0}$ em (2.10), podem ser tomadas independentes de $d_{i} \geq 1, i=1,2, \cdots, n$.

Lema 4.2 Seja $\phi=\left(\phi_{1}, \phi_{2}, \cdots, \phi_{n}\right) \in H^{1}\left(\Omega, \mathbb{R}^{n}\right)$ uma solução fraca do problema elíptico

$$
\begin{aligned}
& -D \Delta \phi+C_{0} \phi=C_{1} \quad \text { em } \Omega \text {, } \\
& D \frac{\partial \phi}{\partial \vec{n}}+B_{0} \phi=B_{1} \quad \text { em } \Gamma \text {, }
\end{aligned}
$$

onde $C_{0}, C_{1}, B_{0}$ e $B_{1}$ são dados em (2.10), d é dado em (4.12) e $\lambda_{1}>0$ é o primeiro autovalor de (4.14). Se $d \geq 1$, então

$$
\|\phi\|_{L^{\infty}\left(\Omega, \mathbb{R}^{n}\right)} \leq K,
$$

onde $K=K\left(\Omega, \Gamma, C_{0}, B_{0}, C_{1}, B_{1}, N, \lambda_{1}\right)$ é uma constante positiva $e$ independente de $d \geq 1$.

Demonstraçāo: Ao longo desta prova, fixaremos $i \in\{1,2, \cdots, n\}$. Mostremos inicialmente que

$$
\left\|\phi_{i}\right\|_{L^{2}(\Omega)} \leq K_{1},
$$

onde $K_{1}=K_{1}\left(\Omega, \Gamma, B_{1}, B_{0}, C_{1}, C_{0}, \lambda_{1}\right)$ e $\phi_{i}$ é a solução do problema

$$
\begin{aligned}
& -d_{i} \triangle \phi_{i}+C_{0} \phi_{i}=C_{1} \quad \text { em } \Omega, \\
& d_{i} \frac{\partial \phi_{i}}{\partial \vec{n}}+B_{0} \phi_{i}=B_{1} \quad \text { em } \Gamma .
\end{aligned}
$$


Multiplicando a primeira equação de (4.17) por $\phi_{i}$ e integrando por partes, obtemos que

$$
d_{i} \int_{\Omega}\left|\nabla \phi_{i}\right|^{2}+C_{0} \int_{\Omega} \phi_{i}^{2}-C_{1} \int_{\Omega} \phi_{i}+B_{0} \int_{\Gamma} \phi_{i}^{2}-B_{1} \int_{\Gamma} \phi_{i}=0 .
$$

Tomando $\psi=\phi_{i}-\frac{B_{1}}{2 B_{0}}$, e usando o fato que $\lambda_{1}\left(d_{i}\right)>\lambda_{1}>0$, onde $\lambda_{1}\left(d_{i}\right)$ é o primeiro autovalor do problema linear associado à (4.17), temos que

$$
\begin{aligned}
& \lambda_{1}\left(d_{i}\right) \int_{\Omega} \psi^{2}-C_{1} \int_{\Omega} \psi+\frac{C_{0} B_{1}}{B_{0}} \int_{\Omega} \psi \\
& \leq \int_{\Omega} d_{i}|\nabla \psi|^{2}+C_{0} \int_{\Omega} \psi^{2}+B_{0} \int_{\Gamma} \psi^{2}+\left(\frac{C_{0} B_{1}}{B_{0}}-C_{1}\right) \int_{\Omega} \psi
\end{aligned}
$$

substituindo o valor de $\psi$ e usando (4.18), segue que

$$
\lambda_{1}\left(d_{i}\right) \int_{\Omega} \psi^{2}+K_{2} \int_{\Omega} \psi+K_{3} \leq 0
$$

onde

$$
K_{2}:=-C_{1}+\frac{C_{0} B_{1}}{B_{0}} \text { e } \quad K_{3}:=|\Gamma| \frac{B_{0} B_{1}^{2}}{4 B_{0}^{2}}+\left(\frac{C_{1} B_{1}}{2 B_{0}}-\frac{3}{4} \frac{C_{0} B_{1}^{2}}{B_{0}^{2}}\right)|\Omega| \text {. }
$$

Mas, a Desigualdade de Young implica que para todo $\varepsilon>0$,

$$
-K_{2} \int_{\Omega} \psi-K_{3} \leq\left|K_{3}\right|+\left|K_{2}\right||\Omega| \varepsilon / 2+\frac{\left|K_{2}\right|}{2 \varepsilon} \int_{\Omega}|\psi|^{2}
$$

Logo, como $d_{i} \geq 1$, segue do Lema 4.1 que

$$
\left(\lambda_{1}-\frac{\left|K_{2}\right|}{2 \dot{\varepsilon} \cdot}\right) \int_{\Omega}|\psi|^{2} \leq\left(\lambda_{1}\left(d_{i}\right)-\frac{\left|K_{2}\right|}{2 \varepsilon}\right) \int_{\Omega}|\psi|^{2} \leq\left|K_{3}\right|+\frac{\left|K_{2}\right||\Omega|}{2} \varepsilon,
$$

onde $\lambda_{1}$ é o primeiro autovalor de (4.14). Tomando $\varepsilon=\left|K_{2}\right| / \lambda_{1}$, obtemos que

$$
\|\psi\|_{L^{2}(\Omega)} \leq \frac{1}{\lambda_{1}} \sqrt{2\left|K_{3}\right| \sqrt{\sqrt{\lambda_{1}}}+\left|K_{2}\right|^{2}|\Omega|}
$$

e, portanto,

$$
\left\|\phi_{i}\right\|_{L^{2}(\Omega)} \leq K_{1}
$$

onde $K_{1}=K_{1}\left(\Omega, \Gamma, C_{0}, C_{1}, B_{0}, B_{1}, \lambda_{1}\right)>0$. 
Vamos agora mostrar a limitação em $L^{\infty}(\Omega)$. Suponhamos que $\frac{2 N}{N+2} \leq p<\frac{N}{2}$. Consideremos

$$
r:=\frac{(N-2) p}{N-2 p} \geq 2 \quad \text { e } \quad k \geq 1
$$

Definamos

$$
\phi=\left(\phi_{i}-k\right)^{+}
$$

Multiplicando a primeira equação de (4.17) por $|\phi|^{r-2} \phi$ e integrando por partes, obtemos que

$$
\begin{aligned}
& \left.\left.\frac{4(r-1)}{r^{2}} \int_{\Omega} d_{i}|\nabla| \phi\right|^{r / 2}\right|^{2}+C_{0} \int_{\Omega}\left(|\phi|^{r / 2}\right)^{2}+B_{0} \int_{\Gamma}\left(|\phi|^{r / 2}\right)^{2} \\
& =\int_{\Omega}\left(C_{1}-k C_{0}\right)|\phi|^{r-2} \phi+\int_{\Gamma}\left(B_{1}-k B_{0}\right)|\phi|^{r-2} \phi
\end{aligned}
$$

Chamando

$$
\tilde{C}:=C_{1}-k C_{0} \quad \text { e } \quad \tilde{B}:=B_{1}-k B_{0}
$$

a Desigualdade de Holder implica que

$$
\begin{aligned}
& \left.\left.\frac{4(r-1)}{r^{2}} \int_{\Omega} d_{i}|\nabla| \phi\right|^{r / 2}\right|^{2}+C_{0} \int_{\Omega}\left(|\phi|^{r / 2}\right)^{2}+B_{0} \int_{\Gamma}\left(|\phi|^{r / 2}\right)^{2} \\
& \leq|\tilde{C}||\Omega|^{1 / p}\left\||\phi|^{r / 2}\right\|_{L^{2 p^{\prime} / r^{\prime}(\Omega)}}^{2 / r^{\prime}}+\left.\left|\tilde{B}\left\|\left.\Gamma\right|^{\frac{N-p}{(N-1) p}}\right\|\right| \phi\right|^{r / 2} \|_{L^{2 / p^{\prime}(N-1)}}^{2 / r^{\prime}}(\Gamma)
\end{aligned}
$$

fazendo $v:=|\phi|^{r / 2}$ e lembrando que $p^{\prime} / r^{\prime}=N /(N-2)$, obtemos que

$$
\begin{aligned}
& \frac{4(r-1)}{r^{2}} \int_{\Omega} d_{i}|\nabla v|^{2}+\left(C_{0}+\lambda\right) \int_{\Omega}|v|^{2}+B_{0} \int_{\Gamma}|v|^{2} \\
& \leq|\bar{C}|\|v\|_{L^{2 \frac{N}{2}(\Omega)}}^{2 / r^{\prime}}+|\bar{B}|\|v\|_{L^{\frac{2(N-1)}{N-2}(\Gamma)}}^{2 / r^{\prime}}+\lambda \int_{\Omega} v^{2}
\end{aligned}
$$

onde $\bar{C}=|\tilde{C}||\Omega|^{1 / p}$ e $\bar{B}=|\tilde{B}| \mid \Gamma^{\frac{N-p}{(N-1) p}}$.

Agora de (1.54) e (1.55),

$$
\begin{aligned}
& H^{1}(\Omega) \hookrightarrow L^{2 N /(N-2)}(\Omega) \\
& H^{1}(\Omega) \hookrightarrow L^{2(N-1) /(N-2)}(\Gamma),
\end{aligned}
$$


com constante de imersão $C=C(\Omega, \Gamma, N)$. Então

$$
\begin{aligned}
& \frac{4(r-1)}{r^{2}} \int_{\Omega} d_{i}|\nabla v|^{2}+\left(C_{0}+\lambda\right) \int_{\Omega}|v|^{2}+B_{0} \int_{\Gamma}|v|^{2} \\
& \leq C|\bar{C}|\|v\|_{H^{1}(\Omega)}^{2 / r^{\prime}}+C|\bar{B}|\|v\|_{H^{1}(\Omega)}^{2 / r^{\prime}}+\lambda \int_{\Omega} v^{2} .
\end{aligned}
$$

Escolhamos $\lambda=\lambda\left(\Omega, B_{0}, C_{0}\right)>0$ tal que

$$
\frac{4(r-1)}{r^{2}} \int_{\Omega} d_{i}|\nabla v|^{2}+\left(C_{0}+\lambda\right) \int_{\Omega}|v|^{2}+B_{0} \int_{\Gamma}|v|^{2} \geq \frac{4(r-1)}{r^{2}} d_{i}\|v\|_{H^{2}(\Omega)^{2}}^{2} .
$$

Assim,

$$
\begin{aligned}
\frac{(r-1)}{r^{2}} d_{i}\|v\|_{H^{1}(\Omega)^{2}}^{z_{0}} & \leq(C|\bar{C}|+C|\bar{B}|)\|v\|_{H^{1}(\Omega)}^{2 / r^{\prime}}+\lambda \int_{\Omega} v^{2} \\
& \leq(C|\bar{C}|+C|\bar{B}|)\|v\|_{H^{1}(\Omega)}^{2 / r^{\prime}}+\lambda\|v\|_{L^{2}(\Omega)}^{2 / r}\|v\|_{H^{1}(\Omega)}^{2 / r^{\prime}}
\end{aligned}
$$

daí,

$$
\frac{r-1}{r^{2}} d_{i}\|v\|_{H^{1}(\Omega)}^{2 / r} \leq C|\bar{C}|+C|\bar{B}|+\lambda\|v\|_{L^{2}(\Omega)}^{2 / r} .
$$

Como $d_{i} \geq 1$, então

$$
\begin{aligned}
\frac{r-1}{r^{2}}\|\phi\|_{L^{N p /(N-2 p)}(\Omega)} & =\frac{r-1}{r^{2}}\|v\|_{L^{2 N /(N-2)}(\Omega)}^{2 / r} \\
& \leq \frac{r-1}{r^{2}} d_{i}\|v\|_{H^{1}(\Omega)}^{2 / r} \\
& \leq C\left(C|\bar{C}|+C|\bar{B}|+\lambda\|v\|_{L^{2}(\Omega)}^{2 / r}\right) \\
& =C\left(C|\bar{C}|+C|\bar{B}|+\lambda\|\phi\|_{L^{r}(\Omega)}\right)
\end{aligned}
$$

Mas $2 \leq r \leq \frac{N p}{N-2 p}$, então para cada $\varepsilon>0$, existe $C_{\varepsilon}(\Omega, N, p)$ tal que

$$
\|\phi\|_{L^{r}(\Omega)} \leq \varepsilon\|\phi\|_{L^{\frac{N p}{N-2 p}}(\Omega)}+C_{\varepsilon}\|\phi\|_{L^{2}(\Omega)}
$$

Assim,

$$
\left(\frac{r-1}{r^{2}}-\lambda C \varepsilon\right)\|\phi\|_{L^{N p /(N-2 p)(\Omega)}} \leq C\left(C|\bar{C}|+C|\bar{B}|+\lambda C_{\varepsilon}\|\phi\|_{L^{2}(\Omega)}\right) .
$$

Portanto,

$$
\|\phi\|_{L^{N p /(N-2 p)(\Omega)}} \leq K_{4}\left[\left|C_{1}\right|+\left|B_{1}\right|+k+\|\phi\|_{L^{2}(\Omega)}\right]
$$


onde $K_{4}:=K_{4}\left(\Omega, \Gamma, N, p, B_{0}, C_{0}\right)>0$.

$$
q \geqslant \frac{2 N}{N+2}
$$

Agora, para todo

$$
\|\phi\|_{L^{q}(\Omega)} \leq K_{4}\left[\left|C_{1}\right|+\left|B_{1}\right|+k+\left\|\phi_{i}\right\|_{L^{2}(\Omega)}\right],
$$

$\operatorname{com} K_{4}:=K_{4}\left(\Omega, \Gamma, N, q, B_{0}, C_{0}\right)>0$.

Para $k \geq 1$, consideremos o conjunto

$$
A_{k}:=\left\{x \in \Omega: \phi_{i}(x)>k\right\} .
$$

Como acima, para $\phi=\left(\phi_{i}-k\right)^{+}$, existe $\lambda=\lambda\left(\Omega, B_{0}, C_{0}, N\right)>0$ tal que

$$
\int_{\Omega} d_{i}|\nabla \phi|^{2}+\int_{\Gamma} B_{0} \phi^{2}+\int_{\Omega}\left(C_{0}+\lambda\right) \phi^{2} \geq d_{i}\|\phi\|_{H^{1}(\Omega)}^{2} \geq\|\phi\|_{H^{1}(\Omega)}^{2},
$$

onde a última desigualdade segue do fato que $d_{i} \geq 1$. Ainda, multiplicando a primeira equação de (4.17) por $\phi$ e integrando por partes, obtemos que

$$
\int_{\Omega} d_{i}|\nabla \phi|^{2}+\int_{\Gamma} B_{0} \phi^{2}+\int_{\Omega}\left(C_{0}+\lambda\right) \phi^{2}=\int_{\Omega}\left(C_{1}-k C_{0}\right) \phi+\int_{\Gamma}\left(B_{1}-k B_{0}\right) \phi+\lambda \int_{\Omega} \phi^{2}
$$

Portanto, (4.20) e (4.21) implicam que

$$
\begin{aligned}
\left(\|\phi\|_{L^{1}(\Omega)}\left|A_{k}\right|^{-\frac{N+2}{2 N}}\right)\|\phi\|_{H^{1}(\Omega)} & \leq\|\phi\|_{L}{\frac{2 N}{N^{-2}}(\Omega)}\|\phi\|_{H^{1}(\Omega)} \\
& \leq C\|\phi\|_{H^{1}(\Omega)}^{2} \\
& \leq C\left(\int_{\Omega}\left|C_{1}-k C_{0}\left\|\phi\left|+\int_{\Gamma}\right| B_{1}-k B_{0}\right\| \phi\right|+\lambda \int_{\Omega}|\phi|^{2}\right)
\end{aligned}
$$

onde $C=C(\Omega, \Gamma, N)$. Estimando cada um dos termos do segundo membro da desigualdade acima, segue que

$$
\begin{aligned}
& \int_{\Omega}\left|C_{1}-k C_{0}\left\|\phi|\leq| C_{1}-\left.k C_{0}\left|C\|\phi\|_{H^{1}(\Omega)}\right| A_{k}\right|^{\frac{1}{2}}\right.\right. \\
& \int_{\Gamma}\left|B_{1}-k B_{0}\right||\phi| \leq\left|B_{1}-k B_{0}\right| C\|\phi\|_{H^{1}(\Omega)}\left|A_{k}\right|^{\frac{1}{2}}
\end{aligned}
$$

e, finalmente

$$
\begin{aligned}
\lambda \int_{\Omega}|\phi|^{2} & \leq \lambda\|\phi\|_{L^{q}(\Omega)}\|\phi\|_{L^{\frac{2 N}{N^{2}}(\Omega)}}\left|A_{k}\right|^{\frac{1}{2}+\frac{1}{N}-\frac{1}{q}} \\
& \leq \lambda C\|\phi\|_{L^{q}(\Omega)}\|\phi\|_{H^{1}(\Omega)}\left|A_{k}\right|^{\frac{1}{2}+\frac{1}{N}-\frac{1}{q}}
\end{aligned}
$$


tomando $q=2 N$ e usando (4.19), segue que

$$
\lambda \int_{\Omega}|\phi|^{2} \leq \lambda C K_{4}\left(\left|C_{1}\right|+\left|B_{1}\right|+k+\left|\phi_{i}\right|_{L^{2}(\Omega)}\right)\|\phi\|_{H^{1}(\Omega)}\left|A_{k}\right|^{1 / 2+1 / 2 N},
$$

$\operatorname{com} K_{4}=K_{4}\left(\Omega, \Gamma, B_{0}, C_{0}, N\right)$.

Portanto,

$$
\left(\|\phi\|_{L^{1}(\Omega)}\left|A_{k}\right|^{-\frac{N+2}{2 N}}\right)\|\phi\|_{H^{1}(\Omega)} \leq\left(C | A _ { k } | ^ { 1 / 2 } \left(\left(\left|C_{1}+k\right| C_{0} \mid\right)+\left(\left|B_{1}+k\right| B_{0} \mid\right)\right.\right.
$$

$$
\left.\left.+\lambda C\left|A_{k}\right|^{1 / 2 N}\left(\left|C_{1}\right|+\left|B_{1}\right|+k+\left\|\phi_{i}\right\|_{L^{2}(\Omega)}\right)\right)\right)\|\phi\|_{H^{1}(\Omega)},
$$

ou seja,

$$
\|\phi\|_{L^{1}(\Omega)} \leq\left|A_{k}\right|^{1+\varepsilon} K_{5} k\left(\left|C_{1}\right|+\left|B_{1}\right|+k+\left\|\phi_{i}\right\|_{L^{2}(\Omega)}\right),
$$

onde $K_{5}=K_{5}\left(\Omega, \Gamma, B_{0}, C_{0}, N\right)>0, \lambda=\lambda\left(\Omega, B_{0}, C_{0}, N\right)>0$ e $\varepsilon=\varepsilon(N)$.

Logo, o Lema 3.4 implica que

$$
\max \left\{\phi_{i}(x) ; x \in \Omega\right\} \leq K_{6}\left(\left|C_{1}\right|+\left|B_{1}\right|+\left\|\phi_{i}\right\|_{L^{2}(\Omega)}\right),
$$

onde $K_{6}=K_{6}\left(\Omega, \Gamma, C_{0}, B_{0}, N\right)$. Trocando $\phi_{i}$ por $-\phi_{i}$, com um argumento análogo, otemos que

$$
\|\phi\|_{L^{\infty}(\Omega)} \leq K_{6}\left(\left|C_{1}\right|+\left|B_{1}\right|+\left\|\phi_{i}\right\|_{L^{2}(\Omega)}\right),
$$

A primeira parte deste lema implica que

$$
\|\phi\|_{L^{\infty}(\Omega)} \leq K,
$$

onde $K=K\left(\Omega, \Gamma, B_{0}, B_{1}, C_{0}, C_{1}, N, \lambda_{1}\right)>0$, e $\lambda_{1}$ é o primeiro autovalor de (4.14), completando a prova do lema.

Finalmente, o próximo resultado garante uma estimativa do atrator $\mathcal{A}_{X}(D)$, uniforme em relação à matriz $D$, se $d_{i} \geq 1, i=1,2, \cdots, n$.

Corolário 4.1 Sejam d como definido em (4.12) e $\alpha$ satisfazendo (4.13). Se $d \geq 1$, então existe $\mathcal{M}>0$ independente de $D$ tal que

$$
\mathcal{A}_{X}(D) \subset \mathcal{B}:=\left\{\phi \in L^{\infty}\left(\Omega, \mathbb{R}^{n}\right) ;\|\phi\|_{L^{\infty}\left(\Omega, \mathbb{R}^{n}\right)} \leq \mathcal{M}\right\},
$$

e atrai subconjuntos limitados de $X$, através do fluxo $T_{D}(t)$ definido em (4.4), na norma de $E_{r}^{\alpha}, 2 \alpha<1+\frac{1}{r}$ er $\geq q$. 
Demonstração: Os Lemas 4.1, 4.2 e o item (ii) do Teorema 2.8 demonstram o corolário.

Lema 4.3 Sejam $F, G: \mathbb{R}^{n} \rightarrow \mathbb{R}^{n}$ funções como definidas em (2.8) e satisfazendo as condições do Capítulos 1 e 2. Consideremos a decomposição de $E^{\alpha}$ dada em (4.5) e

$$
\begin{aligned}
& P: U+U_{\alpha}^{\perp} \rightarrow U \\
& Q: U+U_{\alpha}^{\perp} \rightarrow L^{q}\left(\Omega, \mathbb{R}^{n}\right),
\end{aligned}
$$

definidas em (4.8). Suponhamos que $\alpha$ er satisfaçam

$$
\alpha>1 / 2 q, \quad \alpha+r<1 \quad \text { e } \quad \frac{1}{2 q^{\prime}}<r<\frac{N}{2 q^{\prime}},
$$

Definamos $H: U+U_{\alpha}^{\perp} \rightarrow E_{q}^{-r}$, atuando em funções $\phi \in E_{q^{\prime}}^{r}$ da seguinte forma:

$$
(H(v, w), \phi\rangle=\int_{\Omega} Q(v+w(x)) \phi(x) d x+\int_{\Gamma} G(v+w(y)) \phi(y) d y .
$$

Então, fixado $R>0$ arbitrário, existem constantes positivas $N_{H}, L_{H}, N_{P}, L_{P}$ tal que

$$
\begin{aligned}
\|H(v, w)\|_{E_{q}^{-r}} & \leq N_{H} \\
\left\|H\left(v_{1}, w_{1}\right)-H\left(v_{2}, w_{2}\right)\right\|_{E_{q}^{-r}} & \leq L_{H}\left(\left|v_{1 \boldsymbol{r}} v_{2}\right|+\left\|w_{1}-w_{2}\right\|_{U_{\alpha}^{\perp}}\right) \\
|P(v, w)|_{\mathbb{R}^{n}} & \leq N_{P} \\
\left|P\left(v_{1}, w_{1}\right)-P\left(v_{2}, w_{2}\right)\right|_{\mathbb{R}^{n}} & \leq L_{P}\left(\left|v_{1}, v_{2}\right|+\left\|w_{1}-w_{2}\right\|_{U_{\alpha}^{\perp}}\right),
\end{aligned}
$$

para todo $\left\|v_{1}+w_{1}\right\|_{E^{\alpha}},\left\|v_{2}+w_{2}\right\|_{E^{\alpha}},\|v+w\|_{E^{\alpha}} \leq R$.

Demonstração: Sejam $u_{1}=v_{1}+w_{1}, u_{2}=v_{2}+w_{2}, u=v+w \in E^{\alpha}$, satisfazendo as condições do enunciado deste lema. Temos

$$
\begin{aligned}
|P(v, w)|_{\mathbb{R}^{n}} & =\left|\int_{\Omega} F(v+w(x)) d x+\int_{\Gamma} G_{\Gamma}(v+w(y)) d y\right|_{\mathbb{R}^{n}} \\
& \leq \int_{\Omega}|F(v+w(x))|_{\mathbb{R}^{n}} d x+\int_{\Gamma}|G(v+w(y))|_{\mathbb{R}^{n}} d y \\
& \leq\left\|F_{\Omega}(v+w)\right\|_{L^{q}\left(\Omega, \mathbb{R}^{n}\right)}+|\Gamma|^{1 / q^{\prime}} \\
& \leq N_{P}
\end{aligned}
$$


onde

$$
N_{P}:=N_{F}(R, q, N)+K(\Gamma, q, N) N_{G}(R, q, N)
$$

e para toda $\phi \in E_{q^{\prime}}^{r}$

$$
\begin{aligned}
& |\langle H(v, w), \phi\rangle|=\left|\int_{\Omega} Q(v+w(x)) \phi(x) d x+\int_{\Gamma} G(v+w(y)) \phi(y) d y\right| \\
& =\left|\int_{\Omega}(F(v+w(x))-P(v, w)) \phi(x) d x+\int_{\Gamma} G(v+w(y)) \phi(y) d y\right| \\
& \leq\left(\left\|F_{\Omega}(v+w)\right\|_{L^{q}\left(\Omega, \mathbb{R}^{n}\right)}+|P(v, w)|_{\left.\mathbb{R}^{N}\right)}\|\phi\|_{L^{q^{\prime}}\left(\Omega, \mathbb{R}^{n}\right)}\right. \\
& +\left\|G_{\Gamma}(v+w)\right\|_{L^{q}\left(\Gamma, \mathbb{R}^{n}\right)}\|T(\phi)\|_{L a^{\prime}\left(\Gamma, \mathbb{R}^{n}\right)}
\end{aligned}
$$

onde $T$ denota o operador traço. Agora, com os valores de $\alpha$ e $r$ dados em (4.22), (1.35) e (1.55) implicam que

$$
\begin{aligned}
|\langle H(v, w), \phi\rangle| & \leq\left(N_{F}(R, q, N)+N_{P}+N_{G}(R, q, N) K(r, q, N, \Omega, \Gamma)\right)\|\phi\|_{E_{q^{\prime}}^{r}} \\
& \leq N_{H}\|\phi\|_{E_{q^{\prime}}^{r}} .
\end{aligned}
$$

logo,

$$
\|H(v, w)\|_{E_{q}^{-r}} \leq N_{H}
$$

onde

$$
N_{H}:=N_{F}(R, q, N)+N_{P}+N_{G}(R, q, N) K(r, q, N, \Omega, \Gamma)
$$

Com os mesmos argumentos, mostrarmos as outras duas desigualdades afirmadas no lema.

Seja $\mathcal{A}_{0}$ o atrator compacto da equação diferencial ordinária (4.9), entãa como em [13], existe $\Sigma: \mathbb{R}^{n} \rightarrow \mathbb{R}$ uma função Lipschitz contínua, com constante de Lipschitz $L_{\Sigma}$, tal que

1. $\Sigma(v)=0$, para todo $v \in \mathcal{A}_{0}$ 
2. $a\left(d\left(v, \mathcal{A}_{0}\right)\right) \leq \Sigma(v) \leq b\left(d\left(v, \mathcal{A}_{0}\right)\right)$, onde $a(r)$ é uma função contínua, não decrescente, $a(r)>0$ se $r>0, a(r) \rightarrow+\infty$ quando $r \rightarrow+\infty$ e $b(r)$ é uma função contínua com $b(0)=0$;

3. Para todo $v \in \mathbb{R}^{n}$,

$$
\dot{\Sigma}_{(4.9)}(v) \leq-\Sigma(v)
$$

onde,

$$
\dot{\Sigma}_{(4.9)}(v)=\limsup _{h \rightarrow 0^{+}} \frac{\Sigma(x(h ; v))-\Sigma(v)}{h},
$$

e $x(\cdot ; v)$ é uma solução da $\operatorname{EDO}(4.9)$, com dado inicial $x(0 ; v)=v$.

Fixemos $\alpha$ e $r$ satisfazendo (4.22). Para todo $c>0$ e $\eta<0$ seja

$$
V_{c}:=\left\{v \in \mathbb{R}^{n} ; \Sigma(v)<c\right\}
$$

A propriedade 2 acima implica que para todo $c>0, \vec{V}_{c}$ é compacto. Ainda, para todo $c>0$ e $\eta>0$, consideremo o conjunto

$$
W_{c, \eta}:=\left\{u=v+w \in E^{\alpha} ; v \in V_{c} \text { e }\|w\|_{E^{\alpha}}<\eta\right\}
$$

Temos o

Lema 4.4 Sejam $u_{0}=v_{0}+w_{0} \in X$, $\alpha$ er satisfazendo (4.22) e $u\left(t, \cdot ; u_{0}\right)$ uma solução de (4.1) com dado inicial $u_{0}$. Se existir $t_{0}>0$ tal que $u\left(t_{0}, \cdot ; u_{0}\right) \in W_{c, \eta}$, então existe $d_{0}>0$, suficientemente grande, tal que

$$
u(t, \cdot):=v(t)+w(t, \cdot) \in W_{c, \eta}
$$

para $t \geq t_{0}$ e $d>d_{0}$, onde $W_{c, \eta}$ é definido em (4.24).

\section{Demonstraçāo:}

Seja $u\left(t, \cdot ; u_{0}\right)$ a solução de (4.1) com dado inicial $u_{0} \in X$. Para $\alpha$ satisfazendo (4.22), consideremos

$$
u(t, \cdot)=v(t)+w(t, \cdot) \in U+U_{\alpha}^{\perp},
$$


onde $v(t) \in \mathbb{R}^{n}$ e $w(t, \cdot) \in U_{\alpha}^{\perp}$, para todo $t>0$. Suponhamos que

$$
v\left(t_{0}\right)+w\left(t_{0}, \cdot\right) \in W_{c, \eta} .
$$

Então, como antes

$$
\left\{\begin{array}{l}
\frac{d}{d t} v(t)=P(v(t), w(t, \cdot)) \quad t>0, \\
\frac{\partial}{\partial t} w(t, x)=D \Delta w(t, x)+Q(v(t), w(t, x)), \quad t>0, x \in \Omega, \\
D \frac{\partial w}{\partial \vec{n}}=G(v(t)+w(t, x)) \quad t>0, x \in \Gamma,
\end{array}\right.
$$

com $P: U+U_{\alpha}^{\perp} \rightarrow U$ e $Q: U+U_{\alpha}^{\perp} \rightarrow L^{q}\left(\Omega, \mathbb{R}^{n}\right)$ definidos como em (4.8). Seja $H: U+$ $U_{\alpha}^{\perp} \rightarrow E_{q}^{-r}$ atuando em funções $\phi \in E_{q^{\prime}}^{r}$ como em (4.16), com $\alpha$ e $r$ satisfazendo (4.22). Podemos escrever o problema (4.25), usando a fórmula da variação das constantes, como

$$
\left\{\begin{array}{l}
\frac{d}{d t} v(t)=F(v(t))+|\Gamma| G(v(t))+[P(v(t), w(t, \cdot))-F(v(t))-|\Gamma| G(v(t))], \quad t>0 \\
v(0)=v_{0} \\
w(t, \cdot)=e^{-A_{D} t} w_{0}+\int_{0}^{t} e^{-A_{D}(t-s)}[H(v(s), w(s, \cdot))] d s, \quad t>0
\end{array}\right.
$$

com $A_{D}$ como definido na Seção 5.1.

Agora, para todo $v \in \mathbb{R}^{n}$ e $w \in U_{\alpha}^{\perp}$, temos

$$
\begin{aligned}
& |P(v, w)-F(v)-| \Gamma|G(v)|=\mid \int_{\Omega} F(v+w(x)) d x \\
& +\int_{\Gamma} G(v+w(y)) d y-F(v)-|\Gamma| G(v) \mid \\
& =\left|\int_{\Omega}[F(v+w(x))-F(v)] d x+\int_{\Gamma}[G(v+w(y))-G(v)] d y\right| \\
& \leq\left\|F_{\Omega}(v+w)-F_{\Omega}(v)\right\|_{L^{q}\left(\Omega, \mathbb{R}^{n}\right)}+|\Gamma|^{1 / q^{\prime}}\left\|G_{\Gamma}(v+w)-G_{\Gamma}(v)\right\|_{L^{q}\left(\Gamma, \mathbb{R}^{n}\right)} \\
& \leq\left(L_{F}+K_{\Omega} L_{\mathbf{G}} \Gamma \mid\right)\|w\|_{E^{\mathrm{a}}} .
\end{aligned}
$$


Assim, para todo $(v(t), w(t, \cdot))$ satisfazendo (4.25) e tal que

$$
v(s) \in V_{c} \text { e }\|w(s, \cdot)\|_{E^{\alpha}}<\eta
$$

para todo $t_{0} \leq s \leq t,(4.26)$ implica que

$$
\begin{aligned}
\dot{\Sigma}(v(t)) & \leq \limsup _{h \rightarrow 0} \frac{\Sigma(v(t+h))-\Sigma(v(t))}{h} \\
& \leq \limsup _{h \rightarrow 0} \frac{\Sigma(v(t+h))-\Sigma(x(t+h ; v(t)))}{h}+\dot{\Sigma}_{(4.9)}(v(t)) \\
& \leq L_{\Sigma} \limsup _{h \rightarrow 0} \frac{|v(t+h)-x(t+h ; v(t))|}{h}-\Sigma(v(t)) \\
& \leq-\Sigma(v(t))+L_{\Sigma}|P(v(t), w(t))-F(v(t))-| \Gamma|G(v(t))| \\
& \leq-\Sigma(v(t))+L_{\Sigma}\left(L_{f}+K_{\Omega} L_{g}|\Gamma|\right)\|w(t, \cdot)\|_{E^{a} .}
\end{aligned}
$$

Ainda, usando as estimativas (4.11) e o Lema 4.3, e fazendo $w(t):=w(t, \cdot)$, obtemos que

$$
\begin{aligned}
\|w(t)\|_{E^{\alpha}}= & \left\|e^{-A_{D} t} w\left(t_{0}\right)+\int_{t_{0}}^{t} e^{-A_{D}(t-s)}[H(v(s), w(s))] d s\right\|_{E^{\alpha}} \\
\leq & K_{1} e^{-(d \delta) t}\left\|w\left(t_{0}\right)\right\|_{E^{\alpha}} \\
& +K_{1} \int_{t_{0}}^{t}(t-s)^{-\alpha-r} e^{-(d \delta)(t-s)}\|H(v(s), w(s))\|_{E_{q}^{-r}} d s \\
= & K_{1} e^{-(d \delta) t}\left\|w\left(t_{0}\right)\right\|_{E^{\alpha}}+K_{1} \int_{t_{0}}^{t}(t-s)^{-\alpha-r} e^{-(d \delta)(t-s)} L_{H}\|w(s)\|_{E^{\alpha}} d s \\
& +K_{1} \int_{t_{0}}^{t}(t-s)^{-\alpha-r} e^{-(d \delta)(t-s)} N_{H} d s .
\end{aligned}
$$


Tomando $0<\sigma<\delta$, e multiplicando a expressão acima por $e^{d \sigma t}$ obtemos que

$$
\begin{aligned}
\left\|w(t) e^{d \sigma t}\right\|_{E^{\alpha}} \leq & K_{1} e^{-d(\delta-\sigma) t}\left\|w\left(t_{0}\right)\right\|_{E^{\alpha}} \\
& +K_{1} N_{H} \int_{t_{0}}^{t}(t-s)^{-\alpha-r} e^{d \sigma t} e^{-(d \delta)(t-s)} d s \\
& +K_{1} L_{H} \int_{t_{0}}^{t}(t-s)^{-\alpha-r} e^{-(d \delta)(t-s)} e^{d \sigma t}\|w(s)\|_{E^{\alpha}} d s \\
\leq & K_{1} e^{-(\delta-\sigma) d t}\left\|w\left(t_{0}\right)\right\|_{E^{\alpha}}+K_{1} N_{H} L e^{d \sigma t} \frac{1}{(d \delta)^{1-\alpha-r}} \\
& +K_{1} L_{H} L y(t) \frac{1}{(d \delta)^{1-\alpha-r}},
\end{aligned}
$$

onde

$$
L:=\int_{0}^{\infty} s^{-\alpha-r} e^{-\left(1-\frac{\sigma}{\delta}\right) s} d s
$$

e

$$
y(t)=\sup \left\{e^{d \sigma s}\|w(s)\|_{E^{\alpha}} ; t_{0} \leq s \leq t\right\}
$$

Fazendo $z(t):=e^{d \sigma t}\|w(t)\|_{E^{\alpha}}, t \geq t_{0}$, obtemos que

$$
z(t) \leq K_{1} e^{-d(\delta-\sigma) t} z\left(t_{0}\right)+\frac{K_{1} N_{H} L e^{d \sigma t}}{(d \delta)^{1-\alpha-r}}+\frac{K_{1} L_{H} L}{(d \delta)^{1-\alpha-r}} y(t)
$$

Se

$$
\xi:=\frac{K_{1} L_{H} L}{(d \delta)^{1-\alpha-r}}
$$

então aplicando o mesmo raciocínio acima para $t_{0} \leq s \leq t$, lembrando que $\delta-\sigma>0$, obtemos que

$$
\begin{aligned}
z(s) & \leq K_{1} e^{-d(\delta-\sigma) s} z\left(t_{0}\right)+\xi y(s)+\frac{K_{1} N_{H} L e^{d \sigma s}}{(d \delta)^{1-\alpha-r}} \\
& \leq K_{1} z\left(t_{0}\right)+\xi y(t)+\frac{K_{1} N_{H} L e^{d \sigma t}}{(d \delta)^{1-\alpha-r}}
\end{aligned}
$$

Logo,

$$
y(t) \leq K_{1} z\left(t_{0}\right)+\xi y(t)+\frac{K_{1} N_{H} L e^{d \sigma t}}{(d \delta)^{1-\alpha-r}}
$$


Como $\alpha+r<1$, existe $d_{0}>0$ suficientemente grande, tal que

$$
1-\xi=1-\frac{K_{1} L_{H} L}{(d \delta)^{1-\alpha-r}}>0
$$

para $d \geq d_{0}$. Assim,

$$
y(t) \leq K_{1}(1-\xi)^{-1}\left[z\left(t_{0}\right)+\frac{N_{H} L e^{d \sigma t}}{(d \delta)^{1-\alpha-r}}\right] .
$$

Resumindo, se $v(s) \in V_{c}$ e $\|w(s)\|_{E^{\alpha}}<\eta, t_{0} \leq s \leq t$, então

$$
\dot{\Sigma}(v(t)) \leq-\Sigma(v(t))+L_{\Sigma}\left(L_{f}+K_{\Omega} L_{g}|\Gamma|\right)\|w(t)\|_{E^{a}}
$$

e

$$
\|w(t)\|_{E^{\alpha}} \leq K_{1}(1-\xi)^{-1}\left[e^{-d \sigma t}\left\|w\left(t_{0}\right)\right\|_{E^{\alpha}}+\frac{N_{H} L}{(d \delta)^{1-\alpha-r}}\right]
$$

Definindo

$$
K_{3}:=\left(L_{f} K_{1}+|\Gamma| K_{\Omega} L_{g}\right) L_{\Sigma}
$$

e

$$
K_{4}(d):=K_{1}(1-\xi)^{-1}\left[e^{-d \sigma t}\left\|w\left(t_{0}\right)\right\|_{E^{\alpha}}+\frac{N_{H} L}{(d \delta)^{1-\alpha-r}}\right],
$$

segue, para $t_{0} \leq s \leq t$, que

$$
\|w(t)\|_{E^{\alpha}} \leq K_{4}(d)
$$

e

$$
\dot{\Sigma}(v(t)) \leq-\Sigma(v(t))+K_{3} K_{4}(d)
$$

Usando o fato que $1-\alpha-r>0$, podemos tomar $d_{0}>0$, suficientemente grande, tal que

$$
c-K_{3} K_{4}(d)>0
$$

e

$$
K_{4}(d)<\eta
$$


para todo $d \geq d_{0}$. Portanto, (4.27) e (4.28) implicam que se $v\left(t_{0}\right) \in V_{c}$ e $\left\|w\left(t_{0}\right)\right\|_{E^{\alpha}}<\eta$, então

$$
v(t) \in V_{c} \text { e }\|w(t)\|_{E^{\alpha}}<\eta
$$

para todo $t \geq t_{0}$ e $d \geq d_{0}$, o que concluí a prova do lema.

Definamos agora o conjunto

$$
\mathcal{K}:=\left\{\phi \in X ;\|\phi\|_{X} \leq K\right\}
$$

O Teorema 2.8 implica que existem constantes positivas $c, \eta$ e $t_{0}$ tais que para todo $u_{0} \in \mathcal{K}$,

$$
u\left(t, \cdot ; u_{0}\right) \in W_{c, \eta}
$$

onde $W_{c, \eta}$ é definido em (4.24). O Lema 4.4 implica que

$$
\gamma^{+}\left(W_{c, \eta}\right) \subset W_{c, \eta}
$$

e é précompacta, para todo $c>0$. Também, o Lema 4.4 mostrou que para $t \geq t_{0}$,

$$
\|w(t)\|_{E^{\alpha} \rightarrow 0,} \text { quando } d \rightarrow+\infty .
$$

Mas, se $v \in \mathcal{A}_{0}$, então $\Sigma(v)=0$. Logo, $W_{c, \eta}$ é uma vizinhança limitada de $\mathcal{A}_{0}$ e existe uma vizinhança, $\mathcal{N}\left(\mathcal{A}_{0}\right)$, de $\mathcal{A}_{0}$ tal que

$$
W_{c, \eta} \subset \mathcal{N}\left(\mathcal{A}_{0}\right)
$$

e

$$
\omega\left(W_{c, \eta}\right) \subset \mathcal{N}\left(\mathcal{A}_{0}\right)
$$

Portanto, aumentando $\eta>0$ e $c>0$, se necessário, de tal forma que

$$
\mathcal{B} \subset W_{c, \eta}
$$

onde $\mathcal{B}$ é dado no Corolário 4.1 , obtemos que para todo $d>d_{0}$, o atrator global compacto, $\mathcal{A}_{X}(D)$, de (4.1) satisfaz .

$$
\mathcal{A}_{X}(D) \subset \mathcal{N}\left(\mathcal{A}_{0}\right)
$$

O próximo lema mostra a existência da variedade invariante afirmada no Teorema 4.1 . 
Lema 4.5 Para todo $\alpha$ er fixado como em (4.22), consideremos a decomposição de $E^{\alpha}$ como em (4.5) e as funções $P: U+U_{\alpha}^{\perp} \rightarrow U$ e $H: U+U_{\alpha}^{\perp} \rightarrow E_{q}^{-r}$, definidas no Lema 4.3 , satisfazendo

$$
\begin{aligned}
\|H(v, w)\|_{E_{q}^{-r}} & \leq N_{H} \\
\left\|H\left(v_{1}, w_{1}\right)-H\left(v_{2}, w_{2}\right)\right\|_{E_{q}^{-r}} & \leq L_{H}\left(\left|v_{1}, v_{2}\right|+\left\|w_{1}-w_{2}\right\|_{U_{\alpha}^{\perp}}\right) \\
|P(v, w)|_{\mathbb{R}^{n}} & \leq N_{P} \\
\left|P\left(v_{1}, w_{1}\right)-P\left(v_{2}, w_{2}\right)\right|_{\mathbb{R}^{n}} & \leq L_{P}\left(\left|v_{1}, v_{2}\right|+\left\|w_{1}-w_{2}\right\|_{U_{\alpha}^{1}}\right),
\end{aligned}
$$

para todo $\left\|v_{1}+w_{1}\right\|_{E^{\alpha}},\left\|v_{2}+w_{2}\right\|_{E^{\alpha}},\|v+w\|_{E^{\alpha}} \leq R$. Supo nhamos também que

$$
\begin{aligned}
& \left\|e^{-A_{D} t} w\right\|_{E^{\alpha}} \leq K_{1} e^{-(d \delta) t}\|w\|_{E^{\alpha}}, w \in U_{\alpha}^{\perp}, t \geq 0 \\
& \left\|e^{-A_{D} t} w\right\|_{E^{\alpha}} \leq K_{1} e^{-(d \delta) t} t^{-\alpha-r}\|w\|_{E_{q}^{-r}}, w \in U_{\delta_{2}, t}^{\perp}>0,
\end{aligned}
$$

para $K_{1}>0$, e $\delta>0, \alpha$ er satisfazendo (4.22). Considere o problema

$$
\left\{\begin{array}{l}
\frac{d}{d t} v(t)=P(v(t), w(t, \cdot)), \quad t>0, \\
\dot{w}\left(t=-A_{D} w(t)+H(v(t), w(t)), \quad t>0 .\right.
\end{array}\right.
$$

Então, para d suficientemente grande, existe uma variedade invariante exponencialmente atratora,

$$
S_{d}:=\left\{(v, w) ; w=\sigma_{d}(v), v \in \mathbb{R}^{n}\right\},
$$

para (4.31), onde $\sigma_{d}: U \rightarrow U_{\alpha}^{\perp}$ sastisfaz

$$
s(d)=\sup _{v \in \mathbb{R}^{n}}\left\|\sigma_{d}(v)\right\|_{U_{\alpha}^{\frac{1}{\alpha}}}
$$

$e$

$$
\left\|\sigma_{d}\left(v_{1}\right)-\sigma_{d}\left(v_{2}\right)\right\|_{U_{\alpha}^{\perp}} \leq l(d)\left|v_{1}-v_{2}\right|_{\mathbb{R}^{n}}
$$

com $s(d), l(d) \rightarrow 0$ quando $d \rightarrow \infty$.

Demonstração: A prova deste lema pode ser encontrada em [17], capítulo 6. 


\section{Referências Bibliográficas}

[1] R. Adams, Sobolev Spaces, Academic Press, New York-London, 1975.

[2] H. Amann, Nonhomogeneous Linear and Quasilinear Elliptic and Parabolic Boundary Value Problems, in: Schmeisser/Triebel: Functions Spaces, Differential Operators and Nonlinear analysis, Teubner Texte zur Mathematik, 133, (1993) 9-126.

[3] H. Amann, Linear and Quasilinear Parabolic Problems. Abstract Linear Theory, Birkhäuser Verlag, Basel, 1995.

[4] J. M. Arrieta, A. N. Carvalho, Abstract Parabolic Problems with Critical Nonlinearities and Applications to Naviers-Stokes and Heat Equations, to appear in the Transactions of the American Mathematical Society.

[5] J. M. Arrieta, A. N. Carvalho, A. Rodrigues Bernal, Parabolic Problems with Nonlinear Boundary Conditions and Critical Nonlinearities, to appear in the Journal of Differential Equations.

[6] J. M. Arrieta, A. N. Carvalho, A. Rodrigues Bernal, Attractors of Parabolic Problems with Nonlinear Boundary Conditions. Uniform Bounds, to appear in the Communications in Partial Differential Equations.

[7] J. Bergh, J. Löfström, Interpolation Spaces. An Introduction, Springer-Verlag, Berlin-New York, 1976.

[8] A. N. Carvalho, J. K. Hale, Large Difusivity With Dispersion, it Nonlinear Analisys, TMA, Vol. $17, \mathrm{n}^{\circ} 12$, (1991) 1139-1151.

[9] A. N. Carvalho, Tomasz Dlotko and H. M. Rodrigues, Upper Semicontinuity of Attractors and Synchronization, J. Math. Anal. Appl., Vol. 220, (1998) 13-41. 
[10] A. N. Carvalho, S. M. Oliva, A. L. Pereira, A. Rodrigues-Bernal, Attractors for Parabolic Problems with Nonlinear Boundary Conditions, J. Math. Anal. Appl., Vol. 207, (1997) 409-461.

[11] R. Courant, D. Hilbert, Methods of Mathematical Physics, Vol. 1, John Wiley \& Sons, New York, 1989.

[12] E. B. Davies, Heat Kernels and Spectral Theory, Cambridge University Press, 1989.

[13] J. K. Hale, Asymptotic Behavior of Dissipative Systems, Mathematical Surveys and Monographs, $\mathrm{n}^{\circ}$ 25, AMS, Providence, RI, 1988.

[14] J. K. Hale, Large Diffusivity and Asymptotic Behavior in Parabolic Systems, J. Math. Analysis Applicable, $\mathrm{n}^{\circ}$ 118, (1986) 455-466.

[15] J. K. Hale, C. Rocha, Varying Boundary Conditions With Large Diffusivity, J. Math. Pures et Applicable, $\mathrm{n}^{\circ}$ 66, (1987) 139-158.

[16] J. K. Hale, C. Rocha, Interaction of Diffusion and Boundary Conditions, Nonlinear Analysis, Vol.11, $\mathrm{n}^{\circ}$ 5, (1987) 633-649.

[17] D. Henry, Geometric Theory of Semilinear Parabolic Problems, Lectures Notes in Mathematics, 840, Springer-Verlag, Berlin-New York, 1981.

[18] O. Ladyzenskaya, N. Ural'tseva, Linear and Quasilinear Elliptic Equations, Academic Press, New York-London, 1968.

[19] V. Maz'ja, Sobolev Spaces, Springer-Verlag, Berlin-New York, 1985.

[20] S. M. Oliva, A. L. Pereira, Attractors for Parabolic Problems with Nonlinear Boundary Conditions in Fractional Powers Spaces Preprint.

[21] A. Pazy, Semigroups of Linear Operators and Applications to Partial Differential Equations, Springer-Verlag, New York, 1983. 